\title{
Scattering in Supersymmetric M(atrix) Models
}

\author{
Dissertation zur Erlangung des akademischen Grades \\ doctor rerum naturalium \\ (Dr. rer. nat.) \\ im Fach Theoretische Physik \\ eingereicht an der \\ Mathematisch-Naturwissenschaftlichen Fakultät I \\ der Humboldt-Universität zu Berlin \\ von \\ Diplomphysiker Robert Helling \\ geboren am 7. April 1973 in Hamburg
}

\begin{abstract}
Amtierender Präsident der Humboldt-Universität zu Berlin Prof. Dr. Dr. h. c. Richard Schröder

Dekan der Mathematisch-Naturwissenschaftlichen Fakultät I Prof. Dr. Bernhard Ronacher
\end{abstract}

Gutachter: Prof. Dr. Hermann Nicolai

Prof. Dr. Dieter Lüst

Dr. habil. Harald Dorn

Tag der Abgabe der Dissertation: 27. April 2000

Tag der mündlichen Prüfung: 25. Juli 2000 


\begin{abstract}
:
In this thesis $\Gamma$ we present several tests of the M(atrix)-Model conjecture that asserts that the dynamics of M-Theory The eleven-dimensional Ur-theory containing all known string theories and also eleven-dimensional supergravity in specific limits $\Gamma$ is given by a quantum mechanical matrix model. In particular $\Gamma$ scattering processes are analyzed both from the M(atrix)-Model and from the supergravity perspective and the corresponding $S$-matrix elements are compared. We find impressive agreement between these two theories as long as only classical supergravity is considered. If one includes also quantum effects on the supergravity side $\Gamma$ the agreement does not persist. In addition to these calculations $\Gamma$ the question of the existence of classical solutions to the M(atrix)-Model equations of motion with momentum transfer is addressed and answered negatively.
\end{abstract}

\title{
Zusammenfassung:
}

In dieser Arbeit stellen wir verschiedene Tests der (M)atrixtheorie-Vermutung vor. Die (M)atrixtheorievermutung besagt $\Gamma$ dass die Dynamik von $\mathrm{M}$-Theorie $\Gamma$ der Urtheorie $\Gamma$ die alle bekannten Stringtheorien und auch elfdimensionale Supergravitation als bestimmte Grenzfälle enthalten soll durch ein quantenmechanisches Matrixmodell gegeben ist. Insbesondere untersuchen wir Streuprozesse sowohl aus Sicht des Matrixmodells $\Gamma$ als auch aus Sicht der Supergravitation $\Gamma$ und vergleichen die resultierenden $S$-Matrixelemente. Wir finden beeindruckende Übereinstimmung zwischen den beiden TheorienГsolange wir uns auf klassische Supergravitation beschränken. Sobald wir auch Quanteneffekte auf der Supergravitationsseite einbeziehen That diese Übereinstimmung keinen Bestand. Des weiteren untersuchen wir die Frage ob Lösungen der klassischen Matrixmodell-Bewegungsgleichungen mit Impulsübertrag existieren $\Gamma$ und finden eine negative Antwort. 


\section{Contents}

1. Contents ................................................... 3

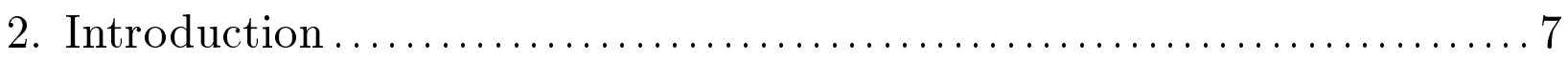

2.1. T-Duality, D-Branes and M-Theory $\ldots \ldots \ldots \ldots \ldots \ldots \ldots \ldots \ldots$

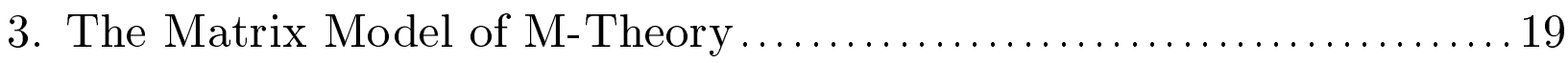

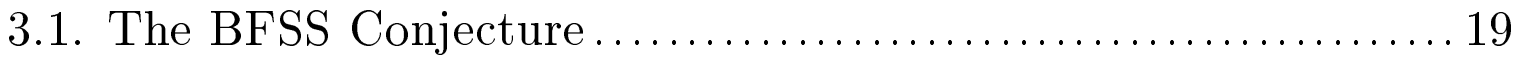

3.2. D0-Particles As Particles...............................23

3.3. T-Duality In The M(atrix)-Model ...................... 25

3.4. The M(atrix)-Model and Supermembranes................27

4. The M(atrix)-Model at One-Loop Order ....................... 31

4.1. The Born-Oppenheimer Effective Potential ................. 31

4.2. The Leading Polarization Independent Effective Potential.....32

4.3. Newton's Law and Coulomb's Law in the Light-Cone Frame... 38

4.4. Spin-Spin Interaction in M(atrix)-Theory ................ 41

4.5. Spin-Spin Interaction in Gravity and String Theory ........... 49

4.6. Understanding the Correspondence ...................... 50

5. Two Loops and Three Particles ............................... 55

5.1. Power-Counting — The Rules of the Game................ 55

5.2. Three-Graviton Scattering at Leading Order ............... 58

5.3. Three Gravitons in M(atrix)-Theory ....................6 64

5.4. Quantum Corrections to Gravity ....................... 71

5.5. Why the Discrepancy? .............................. 80

6. Beyond the Eikonal Regime................................... 89

6.1. Momentum Transfer in Higher Dimensions and $N=\infty \ldots \ldots . .89$

6.2. The Toy-Model.................................... 92

6.3. Scattering Solutions in the M(atrix)-Model ................ 97

7. Conclusions ............................................... 101

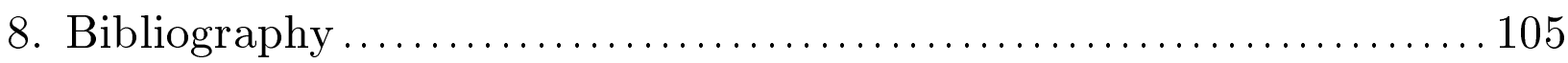



When the objects of an inquiry, in any department, have principles, conditions, or elements, it is through acquaintance with these that knowledge, that is to say scientific knowledge, is attained. For we do not think that we know a thing until we are acquainted with its primary conditions or first principles, and have carried our analysis as far as its simplest elements. Plainly therefore in the science of Nature, as in other branches of study, our first task will be to try to determine what relates to its principles.

The natural way of doing this is to start from the things which are more knowable and obvious to us and proceed towards those which are clearer and more knowable by nature; for the same things are not 'knowable relatively to us' and 'knowable' without qualification. So in the present inquiry we must follow this method and advance from what is more obscure by nature, but clearer to us, towards what is more clear and more knowable by nature. 
1. Contents 


\section{Introduction}

Progress in string theory did not come continously but in several waves. The first $\Gamma$ spanning roughly from 1968 to 1973Гcame after Veneziano's discovery of his famous amplitude[Ve]. It was the time when "dual resonance models" $\Gamma$ as string theory was called during those years $\Gamma$ were supposed to be theories of the strong interactions. From today's point of view $\Gamma$ the open string was an abstraction of the flux tube connecting the two quarks in a meson. Dual resonance models became unpopular as it became clear that they always contain a massless spin two particle that was not wanted from a phenomenological point of view. Even more important Tthere was another much more successful description of strong interactions: Quantum Chromodynamics.

A little later it was recognized that the spin two particle can actually be identified with the graviton $\Gamma$ the particle associated with the metric tensor $\Gamma$ the dynamical field of general relativity. String theory turned into a candidate for a unification of gauge and gravitational interactions. The second wave of popularity of string theory during 1984-1989 (the "first superstring revolution") centered around the unification of gravity with the Standard Model interactions. It brought with it the discovery of the GreenSchwarz anomaly cancellation mechanism and the existence of chiral gauge theories in ten dimensions $\Gamma$ the heterotic string and Calabi-Yau compactifications. Unfortunately $\Gamma$ there seemed to be a proliferation of string vacua and direct contact with the four-dimensional Standard Model physics could not be made.

Since 1995 there is a new wave of string theory activity going on superstring revolution. So farCits main discoveries are (non-perturbative) symmetriesГsocalled dualities $\Gamma$ that map different string theories onto each other $\Gamma$ possibly in different regimes (for example weak and strong coupling). It was realized that the five different superstring theories might not at all be different but stem from a single ancestor $\Gamma \mathrm{M}-$ Theory $\Gamma$ an eleven-dimensional Ur-theory that can be seen to contain all string theories in different limits. Furthermore $\Gamma$ it became clear that strings are not the only dynamical objects in string theories and M-Theory $\Gamma$ at finite values of the string coupling constant $\Gamma$ there are many more objects of different dimensionalities.

In particular $\Gamma$ there are again particles $\Gamma$ the so-called D0-particles $\Gamma$ whose dynamics is given by supersymmetric quantum mechanics of matrix degrees of freedom. In a very influential paper[BFSS] $\Gamma$ Banks $\Gamma F i$ ischler $\Gamma$ Shenker $\Gamma$ and Susskind conjectured that $\Gamma$ in lightcone coordinates $\Gamma$ this matrix quantum mechanics in fact can describe the full dynamics of M-Theory. In this thesis $\Gamma$ we investigate this conjecture and test some of its predictions. In particularГfor low energy processes $\Gamma \mathrm{M}$-Theory should be described by eleven-dimensional supergravity. Here $\Gamma$ we will use the M(atrix)-Model to calculate scattering amplitudes in different kinematic setups and compare them to supergravity $\Gamma$ both in the classical limit and including quantum corrections and find both agreement and disagreement. The disagreement we present here is the first based on an actual scattering calculation reported in the string theory literature. 
This thesis is structured as follows: In the remainder of this introduction $\mathrm{wwe}$ briefly present the results from the second superstring revolution that will be needed later on. In

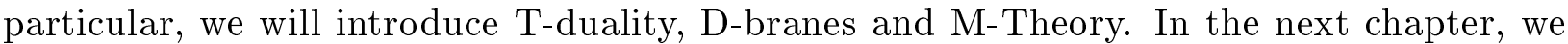
cover the the M(atrix)-Model-conjecture put forward in [BFSS] $\Gamma$ point out the particluarities of the model and some immediate consequences and mention the relation to supermembranes that was already known more than ten years ago.

The following chapter contains some details of how to set up scattering calculations in the M(atrix)-Model $\Gamma$ and how to relate them to supergravity. It covers two particle processes that are described by one loop calculations in M(atrix)-Theory. In particularГit contains a calculation of the D0 spin-spin interactions originally presented in [BHP].

In chapter five $\Gamma$ the analysis is pushed one order further. There $\Gamma$ we present results about three-particle scattering. At the leading two-loop order corresponding to classical supergravity $\Gamma$ again we find full agreement. Then we go one order further and try to match quantum corrections to supergravity with a M(atrix)-Model calculation. There we find the disagreement mentioned above. This is the first disagreement between the two theories reported on in the string theory literature that is based on an actual quantitative calculation. It was first presented in [HPSW].

In the next chapter $\Gamma$ we look at the $\mathrm{M}$ (atrix)-Model equations of motion from a classical perspective. We present a no-go theorem for scattering solutions that use finite dimensional matrices for processes in which momentum is transfered. We end with a chapter containing conclusions.

\subsection{T-Duality, D-Branes and M-Theory}

In this section we are going to give a brief introduction to the notions that came aling with the second superstring revolution. We do not intend to give here a self contained introduction to string theory. Rather $\mathrm{w}$ we assume the reader has a basic understanding of the subject as it can be obtained for example from the first chapter of [GSw]

From the classical perspective $\Gamma$ string theory deals with maps of a two-dimensional world-sheet $\Sigma$ to a target space (space-time) manifold $\mathcal{M}$ :

$$
X: \Sigma \rightarrow \mathcal{M}
$$

The action for such maps in the Polyakov formalism is

$$
\mathcal{S}=-\frac{1}{4 \pi \alpha^{\prime}} \int_{\Sigma} d^{2} \sigma \sqrt{-\gamma} \gamma^{\alpha \beta} G_{\mu \nu}(X) \partial_{\alpha} X^{\mu} \partial_{\beta} X^{\nu}
$$

where $G$ is a metric on $\mathcal{M}$ and $\gamma$ is an (auxiliary) metric on $\sigma$ whose equations of motion turn it into the pull-back $X^{*} G .1 / 4 \pi \alpha^{\prime}$ is the string tension that is used to translate areas 
of the string world sheet into energies. It turns out that for flat targets and with the appropriate choice of gauge $\Gamma$ the equation of motion for such maps $X$ is a free one:

$$
\left(\partial_{\tau}^{2}-\partial_{o}^{2}\right) X=0
$$

If we choose appropriate coordinates $(\tau, \sigma)$ on $\Sigma \Gamma$ this is solved by the ansatz $X(\tau, \sigma)=$ $X_{L}(\tau+\sigma)+X_{R}(\tau-\sigma)$ for arbitrary "left- and right moving" functions $X_{L / R}$.

Let us consider closed strings $\Gamma$ that are strings for which $\Sigma$ has no boundary $\Gamma$ a little closer. We will also assume that topologically $\Sigma=\mathbb{R} \times S^{1}$. If one dimension of the target is also a $S^{1}$ with radius $R \Gamma$ say $\Gamma$ then the quantum mechanical spectrum of the string theory is organized by two quantum numbers: As always for compact directions $\Gamma$ the momentum that is conjugate to the compact dimension is quantized. Its quanta are inversely proportional to the radius $R$. Let us call the momentum quantum number $n$. This is like in ordinary field theories.

String theories enjoy an additional feature as compared to point particle theories: In contrast to particles $\Gamma$ strings can wind around the compact direction. If we parameterize the $S^{1}$ of $\Sigma$ by $\sigma \in[0,2 \pi] \Gamma$ this means that

$$
X(\tau, 0)=X(\tau, 2 \pi)+2 \pi R w
$$

with an integer "winding number" $m$. Since the string's potential energy is given by its length $\Gamma$ such winding states have a potential energy proportional to $w R$. In total $\Gamma$ the mass squared of such a string is given by

$$
m^{2}=\frac{n^{2}}{R^{2}}+\frac{w^{2} R^{2}}{\alpha^{\prime 2}}+\frac{2}{\alpha^{\prime}}(N+\tilde{N}-2)
$$

where $N$ and $\tilde{N}$ are oscillator quantum numbers that will play no rôle here. Note $\Gamma$ that formally this is invariant under interchanging $n$ and $w$ if we simultaneously rescale $R \rightarrow \frac{\alpha^{\prime}}{R}$. This means that using the physics of string theory $\Gamma$ we cannot decide if the radius of compactification is small or large as compared to the string scale

$$
\ell_{s}=\sqrt{\alpha^{\prime}}
$$

This relation between string theories compactified on circles of radii $R$ and $\alpha^{\prime} / R$ is known as T-duality $\Gamma$ where the "T" stands for target space. In terms left and right movers $\Gamma$ it can be shown that this transformation amounts to

$$
X_{R} \mapsto X_{R}, \quad X_{L} \mapsto-X_{L}
$$

This is not only a symmetry of the spectrum but also of the conformal field theories including operator product expansions of the world-volume fields. 
So far $\Gamma$ we have only talked about the bosonic string. In order to describe also fermionic fieldsTone should enlarge the field content of the world-volume field theory and proceed to superstring theory.

There are two supersymmetric string theories with two independent supersymmetries. They are called type IIA and type IIB. Possibly after a gauge fixing (if one employs the Green-Schwarz formalism as we do in most of this thesis) $\Gamma$ there are both a left- and a right-moving space-time fermion. The difference between the two theories is that in IIA the two different supersymmetry charges that come from the left- and right-moving fields respectively carry opposite space-time chiralities whereas in type IIBC the two supersymmetries are of the same chirality. As T-duality flips the sign of one half of the world sheet fields only $\mathrm{Cit}$ can be thought of as a space-time parity operation on the left-movers only. Thus $\Gamma$ it also interchanges type IIA and type IIB. This means $\Gamma$ type IIA compactified on a very small circle is nothing but type IIB compactified on a large circle. For details 5 see [AdWLN]

Let us now consider open strings for which $\Sigma$ has a boundary. This means $\Gamma$ we have to find boundary conditions for the coordinate fields $X$ and their superpartners. For a long time Conly Neumann boundary conditions

$$
\left.\partial_{\sigma} X\right|_{\partial \Sigma}=0
$$

were considered physical since they prohibit momentum flowing of the edge of the string. Note that any boundary condition relates left and right movers. It is now very natural to ask what happens to open strings under T-duality. These Neumann boundary conditions are not preserved under the mapping (2.1) Cbut they turn into Dirichlet boundary conditions

$$
\left.\partial_{\tau} X\right|_{\partial \Sigma}=0 .
$$

This means that in order to obtain a theory that is T self-dual we should consider Neumann and Dirichlet boundary conditions on an equal footing. (2.2) means that in the target space directions where it applies $\Gamma$ the end of the open string is not free to move but is attached to a point given by the initial conditions.

If one imposes Neumann boundary conditions in $(p+1)$ dimensions and Dirichlet conditions in the remaining one $\Gamma$ it is said that the spacetime contains $\mathrm{D} p$-branes or $\mathrm{D}$ branes for short. The end-points of the string move freely in the $p+1$ directions tangential to the $\mathrm{D} p$-brane but cannot get off. In this respect $\Gamma \mathrm{D} p$-branes are topological defects like domain walls in solid state physics.

Although not directly visible after second quantization $\Gamma$ one should imagine a Dbrane covered by open strings. One can scatter strings on on a D-brane by scattering it on the open strings covering the D-branes. By virtue of this process $\Gamma D$-branes become dynamical by themselves and one can attribute oscillations of the open strings to oscillations of the D-brane itself. 


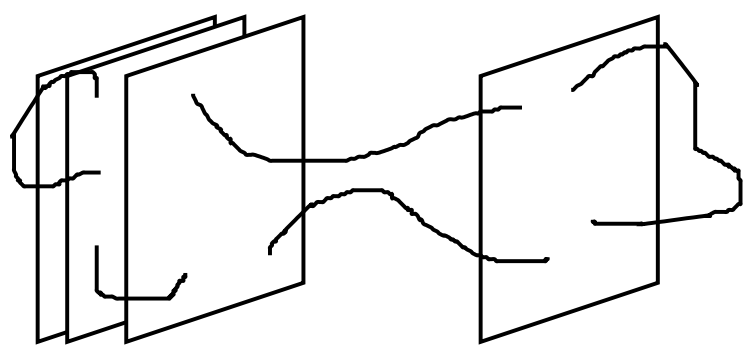

Fig. 1: D2-branes with open strings stretching

The massless modes of open strings have spin one $\Gamma$ hence they are vector fields. Therefore $\Gamma$ the dynamics of the D-brane is also described in terms of these vector fields. In [P1] $\Gamma$ the low energy effective action of a D-brane was found to be given by the Born-Infeld action

$$
\mathcal{S}_{\mathrm{BI}}=\tau_{p} \int d^{p+1} x \sqrt{\operatorname{det}\left(G_{\mu \nu}+\alpha^{\prime} F_{\mu \nu}+B_{\mu \nu}\right)}+\mathrm{WZW-terms}
$$

where $G$ is the pullback of the metric to the world volume of the D-brane $\Gamma B$ is the pullback of the Kalb-Ramond form and $F$ is the field strength of the vector bosons contained in the open string theory. $\tau_{p}$ is the tension of the D-brane that we will determine shortly.

It is important to note that $\Gamma$ in this low energy approximation where the string is basically shrunk to its center of mass $\Gamma$ the vector field only depends on the coordinates tangential to the world volume of the D-brane since the open string it is a part of is glued to the brane at its end-points and thus is the center of mass.

In the limit of large string tension $\Gamma$ in $\mathcal{S}_{\mathrm{BI}}$ Tonly the lowest order term in $F_{\mu \nu}$ survives. Since this is the quadratic term $\Gamma$ this Born-Infeld becomes just the ordinary Maxwell action. This is obtained by dimensional reducing ten-dimensional Maxwell theory to the $(p+1)$ dimensions of the brane's world volume. The components of the gauge field tangential to the world volume transform as vectors under the world volume Lorentz group whereas the components that are normal are scalars from the D-brane world volume perspective.

If there are several D-branesTone introduces labels at the ends of the different open strings determining to which D-brane this end is glued to (note that from this point of view Chan-Patton labels are nothing but D9-branes!). One can imagine a process in which end-points of two open strings that end on the same brane meet and join and then lift of the brane. To make such a process possible the two end-points of a string should transform under conjugate representations of the group that permutes the D-branes.

This tells us that the most general group that accomplishes this is $U(N)$ if we are dealing with a collection of $N \mathrm{D} p$-branes. In this scenario $\Gamma$ the low energy effective action is promoted to a full $U(N)$ Yang-Mills theory living on the world volume of the brane.

The mass (or Tmore appropriate: the tension) of an object is given by the coupling to the graviton field just like the charge is given by the coupling to a gauge field. By interchanging closed strings that contain gravitons as their massless modes between the 
open strings attached to different branes one can compute these couplings and therefore the mass and possibly the charge (under gauge fields in the closed string spectrum) of the D-branes. On dimensional grounds $\Gamma$ we expect the tension to scale like $\alpha^{-\frac{p+1}{2}}$. As the leading order process is given by a cylinder amplitude $\Gamma$ we expect it to contain one inverse power of the string coupling constant and we arrive at

$$
\tau_{p} \propto \frac{1}{g \alpha^{\prime \frac{p+1}{2}}}
$$

In type II theories $\Gamma$ besides the fields that are built from the bosonic $X$ fields $\Gamma$ the dilaton $\Gamma$ the Kalb-Ramond two-form and the metric $\Gamma$ there are form fields $\Gamma$ the so-called Ramond-Ramond fields or RR-fields for short Tarising from tensor products of two spinor fields (in the Green-Schwarz formalism):

$$
C^{\mu_{1} \ldots \mu_{k}}=\bar{\psi}_{L} \Gamma^{\mu_{1} \ldots \mu_{k}} \psi_{R}
$$

In type IIA $\Gamma$ the space-time spinors $\psi_{L / R}$ have opposite chiralities. Therefore $\Gamma$ in type IIA there are forms of odd $k$ whereas in type IIBIthere are forms of even $k$. Fundamental strings are not charged under these fields. Rather Tone would expect objects of $k$-dimensional world volume to couple to these fields via a minimal coupling

$$
\int C
$$

And in fact $\Gamma$ just as D-branes have mass (i.e. couple to the metric) they are also charged under these Ramond-Ramond $k$-form fields. More specifically $\Gamma \mathrm{D} p$-branes are charged under the $(p+1)$-form fields and also under forms of lower degree if the gauge fields on the brane have non-vanishing flux. The coupling is the generalization of electric charge. Furthermore $\Gamma$ with the help of the Hodge operator $\Gamma k$-forms also couple magnetically to $\mathrm{D}(7-k)$-branes.

In what we have said so far $\Gamma$ supersymmetry did not seem to play an important rôle. But this is a misconception Tas it supplies one important additional structure: In all theories with extended supersymmetries $\Gamma$ the algebra contains terms of the general form

$$
\{Q, Q\}=P \pm Z
$$

where we have denoted generic supercharges by $Q \Gamma$ the generator of translations by $P$ and we have included a central charge $Z$. All these objects carry additional indices that are not important here. After sandwiching this relation between some state $\langle\Psi|$ and $|\Psi\rangle$ and using the positivity of $\left\{Q, Q^{\dagger}\right\}$

$$
\langle\Psi|P \pm Z| \Psi\rangle=\langle\Psi|\{Q, Q\}| \Psi\rangle=2 \| Q|\Psi\rangle \|^{2} \geq 0,
$$

we find $\Gamma$ in the rest frame $\Gamma$ the famous "BPS-condition" for the expectation values

$$
M \geq|Z|
$$


This means that the mass is always bounded from below by the charge of a state in appropriate units. Even more important $\Gamma$ if the bound is saturated - the state $\Psi$ is a "BPS-state" - the representation theory of the supersymmetry algebra changes $\Gamma$ there can be multiplet shortening: Multiplets of BPS-states have fewer components than generic multiplets.

As this shortening is a property of the symmetry algebra it survives continuous transformations of the theory like quantization or tuning of the coupling constant. If a theory classically and at weak coupling contains states that saturate the BPS-bound it is generally believed that also the quantum theoryГeven at strong coupling has these states that satisfy the same extremality condition.

It was known for a long time that the low energy supergravity-theories of string theory contain black- $p$-brane solutions $\Gamma$ the higher dimensional generalizations of extremal Reisner-Nordström solutions of four-dimensional Einstein-Maxwell theory $\mathrm{Tth}$ at are extremally charged under the Ramond-Ramond fields. But as fundamental strings are not charged under these fields $\Gamma$ their stringy origin was unclear until Polchinski realized that D-branes also saturate the BPS-bound for the Ramond-Ramond charges and are therefore the much sought for microscopic realizations of these solutions. Specifically $T$ the $\mathrm{D} p$-branes are BPS in type IIA theory for even $p$ whereas for odd $p$ they are BPS in type IIB theory.

Like the extremal Reisner-Nordström solutionГBPS-states often have a linearization property: As they preserve some supersymmetryГsome of the supercharges that square to the Hamiltonian annihilate the state and therefore it is static. Furthermore $\Gamma$ any superposition of several copies of the same BPS state is static: Two extremal RN black hole neither attract nor repel each other since the gravitational attraction is exactly canceled by the electric repulsion. The same happens for D-branes: Parallel D-branes of the same dimension neither attract nor repel since the forces mediated by the graviton $\Gamma$ the dilaton and the Ramond-Ramond field cancel. It is possible for them to form a marginally bound state.

In type IIA theory we expect for $N$ D0-branes such a bound state to have the mass of $N$ single D0-particles. Using (2.3) $\Gamma$ we expect it to be proportional to

$$
m=\frac{N}{g \sqrt{\alpha^{\prime}}} .
$$

Hull $\Gamma$ Townsend[HT] and Witten[W] realized that this looks like the Kaluza-Klein spectrum of an eleven-dimensional theory that is compactified on a circle of radius

$$
R_{11}=g \sqrt{\alpha^{\prime}}
$$

They conjectured that at finite coupling $\Gamma$ type IIA theory is in fact eleven-dimensional. This additional dimension is not visible in perturbation theory since this is an expansion around $g=0$. The eleven-dimensional mother-theory was called M-TheoryГalso to reflect its "mysterious" character. 
The assumption of M-Theory enables one to explain the origin of several features of string theories. For example the type IIB theory that is related to IIA by T-duality on another circle is known to have a $S L(2, \mathbb{Z})$ S-duality $\Gamma$ that can interchange strong and weak coupling and transforms fundamental and D1-strings into each other. This $S L(2, \mathbb{Z})$ symmetry is very natural from the M-perspective: The two circles on which one compactifies M-Theory to reach type IIB form a torus that has a $S L(2, \mathbb{Z})$ modular group that should leave the IIB physics invariant. In particularГsince (2.4) relates the radius and the coupling and the modular group acts on the radii of the two circles that make up a circle $\Gamma$ we find the modular $S L(2, \mathbb{Z})$ acting on the IIB coupling.

M-Theory should have the same 32 supersymmetries as the type IIA string since compactification on circles preserves supersymmetry. It was known from the work of Cremmer and Julia[CJ] that there is only one supersymmetric interacting low-energy theory in eleven dimensions $\Gamma$ namely supergravity with the bosonic Lagrangian given by

$$
\begin{aligned}
\mathcal{L}=- & \frac{1}{2 \kappa^{2}} \sqrt{-g} \mathcal{R}-\frac{1}{8} \sqrt{-g}\left(F_{M N P Q}\right)^{2} \\
& \quad-\frac{\sqrt{3}}{12^{3} \kappa^{3}} \epsilon^{M_{1} \ldots M_{11}} F_{M_{1} M_{2} M_{3} M_{4}} F_{M_{5} M_{6} M_{7} M_{8}} C_{M_{9} M_{10} M_{11}}
\end{aligned}
$$

(for a discussion $\Gamma$ see chapter 4 ).

This eleven-dimensional supergravity gives rise via dimensional reduction to all the maximally extended supergravities in lower dimensions and for some time many people believed in its rôle in a fundamental theory mainly for aesthetical reasons. This hope was shaken when it became clear that also this maximal supergravity is not renormalizable and subject to quantum corrections. Different from the situation in ten dimensions where string theories were at hand to serve as regulating theories that provide the appropriate high energy degrees of freedom $\Gamma$ this seemed not to apply directly to eleven-dimensional supergravity. But with the advent of M-Theory $\mathrm{Mthis}$ attitude changed and it is generally believed that eleven-dimensional supergravity is the low energy description of M-Theory.

The relation between the different Newton constants in Kaluza-Klein dimensional reduction can be deduced from the fact that the gravitational action is always multiplied by a factor of $\kappa_{D}^{-2}$ and that in the course of the compactification the measure of integration is changed as $d^{D} x \mapsto R d^{(D-1)} x$. Therefore $\Gamma$

$$
\frac{1}{\kappa_{11}^{2}}=\frac{R_{11}}{\kappa_{10}^{2}} .
$$

Combining this with the well known relation of the ten-dimensional Newton constant to the string theory parameters $\kappa_{10}=1 / g \ell_{s}^{4} \Gamma$ enables us to express the eleven-dimensional Planck length $\ell_{p}=\kappa_{11}^{2 / 9}$ in terms of ten-dimensional parameters:

$$
\ell_{P}=g^{\frac{1}{3}} \sqrt{\alpha^{\prime}}
$$


This Ttogether with the relation (2.4) constitutes a useful dictionary to translate M-Theory relations to IIA relations and vice versa. It is most often stated as

$$
\alpha^{\prime}=\frac{\ell_{P}^{3}}{R_{11}} \quad g_{s}^{2}=\frac{R_{11}^{3}}{\ell_{P}^{3}}
$$

Note that in eleven uncompactified dimensions $\Gamma$ there is only one constant $\Gamma$ namely the Planck length Tand there are no coupling constants or dimensionless parameters that would allow for a perturbative expansion. This is reflected by the fact that eleven-dimensional supergravity does not have any scalar fields that could play a rôle like the dilaton in ten dimensions. As bosonic fields there are only the metric $g_{M N}$ and a three-form "graviphoton" potential $C_{M N P}$ with field-strength $F_{M N P Q}$. In modern language: M-Theory has no moduli.

The eleven-dimensional supersymmetry algebra reads

$$
\left\{Q_{\alpha}, Q_{\beta}\right\}=\Gamma_{\alpha \beta}^{\mu} P_{\mu}+\Gamma_{\alpha \beta}^{\mu_{1} \mu_{2}} Z_{\mu_{1} \mu_{2}}^{(2)}+\Gamma_{\alpha \beta}^{\mu_{1} \ldots \mu_{5}} Z_{\mu_{1} \ldots \mu_{5}}^{(5)}
$$

As above $\Gamma$ we encounter central charges $\Gamma$ namely a membrane charge $Z^{(2)}$ and a five-brane charge $Z^{(5)}$. It is therefore expected that besides momentum modes ("gravitational waves") that just carry momentum There are fundamental membranes and five-branes in M-Theory that couple electrically respectively magnetically to the graviphoton field. This expectation is fostered by the existence of corresponding BPS solutions to the supergravity equations of motion (see the review of M-Theory stressing the importance of these solutions[T] $)$.

These ingrediences also allow for an explanation of the objects in IIA string theory: The fundamental string is nothing but the eleven-dimensional membrane that wraps around the compact eleventh dimension. As we already found above $\Gamma$ the momentum modes in the compact dimension are via the Kaluza-Klein mechanism to be identified with the D0-particles. The membrane does not have to wrap the compact direction. It can also occur as a membrane in ten dimensions then being interpreted as the D2-brane. The D4-brane arises from the M-Theoryfive-brane wrapping the compact direction whereas the non-wrapping five-brane is known as the Neveu-Schwarz five-brane in ten dimensions.

The higher-dimensional D-branes couple magnetically to the Ramond-Ramond $k$ form fields. Thus they are dual to the branes that we already found eleven-dimensional explanations for. Indeed $\Gamma$ the D6-brane comes as a Kaluza-Klein magnetic monopole that is a Taub-NUT space in eleven dimensions. The D8-brane is difficult to handle since its fields do not decay with radial distance just like the electric field of a charged plane in four dimensions.

There are also relations of M-Theory to the other string theories the heterotic strings. They are not needed in the following and are omitted therefore in this short introduction. 


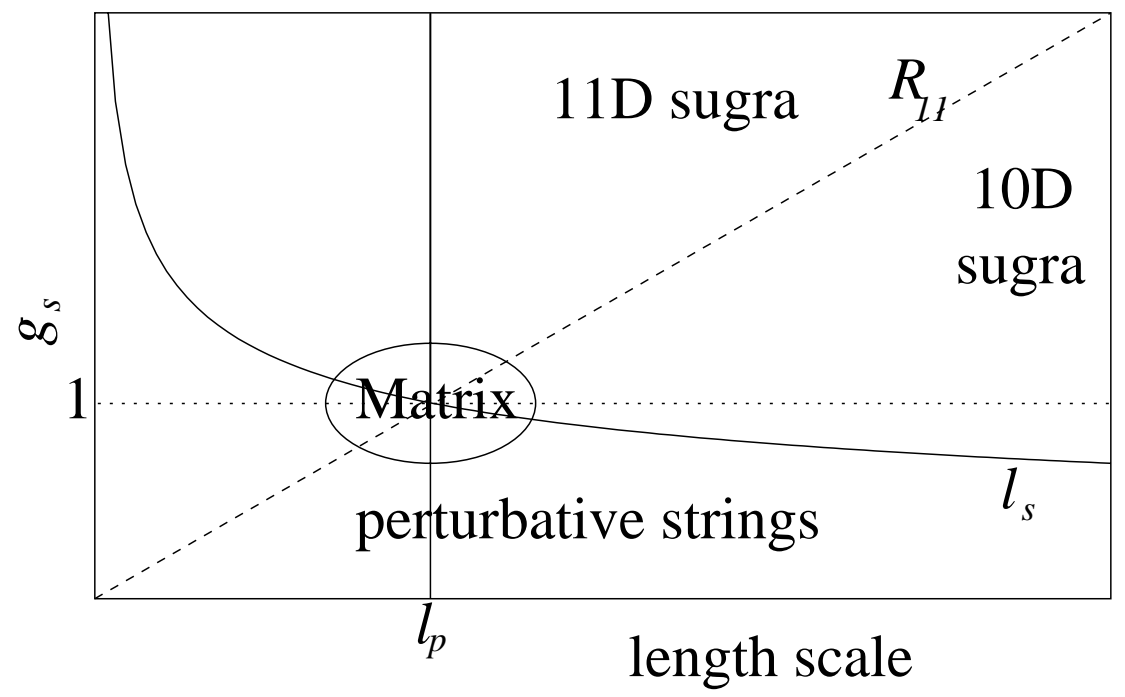

Fig. 2: The M-Theory phase diagram

The situation we find in M-Theory is best displayed in the phase diagram Hermann Verlinde uses to draw.

There are two order parameters: A typical length scale of a physical situation and the string coupling constant. Whenever the coupling is small we expect string perturbation theory to be trustworthy. We have also drawn the different length scales appearing in the dictionary relations (2.5). For length scales larger than the Planck length $\ell_{P} \Gamma$ space-time can be treated classically and the idea of a space-time manifold is valid. Note $\Gamma$ that string theory has a slightly different fundamental length scale $\Gamma$ namely $\ell_{s}$. For processes at larger distances $\Gamma$ we expect classical supergravity to be a good description since higher curvature corrections are suppressed by orders of $\ell_{s}$. Whenever $R_{11}$ is small compared to typical scales of the problem $\Gamma$ the eleventh M-Theory-dimension is not resolved and the world appears to be ten-dimensional. This can be seen to be always the case for perturbative string theory above the Planck scale.

$\mathrm{M}$ (atrix)-Theory appears near the intersection point of all these length scales and it is this thesis' main theme to investigate the overlap with the other regimes and mainly eleven-dimensional supergravity.

We should not end this introduction without pointing the reader to some more literature that can be used as an introduction to the topics covered here. The classic compendium of string theory before the second super-string revolution is the two volume work [GSw]. To us $[\mathbf{L T}]$ has often been very useful. A well written introduction that also covers some of the newer developments and is therefore complementary to the books mentioned so far $\Gamma$ are the two books by Polchinski[P2]. We have already mentioned the review of M-Theory by Townsend that is based on an analysis of the eleven-dimensional superalgebra[T]. There are several reviews of $\mathrm{M}$ (atrix)-Theory. Let us only mention the ones by Bigatti and Susskind[BS] ${ }^{[}$the one by Banks $[\mathbf{B a}]$ and especially the recent one by Taylor with a strong focus on scattering theory[T2]. The lecture notes $[\mathbf{N H}]$ have a strong 
emphasis on the membrane aspect of the M(atrix)-Model. 


\section{The Matrix Model of M-Theory}

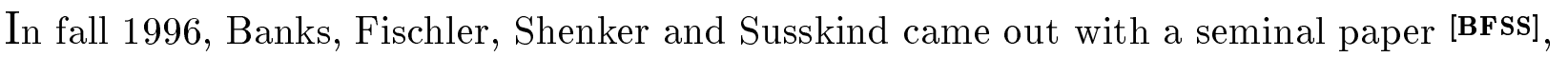
that proposed to give a non-perturbative definition of M-Theory in terms of supersymmetric quantum mechanics of $s u(N)$ matrices. This thesis is concerned with consistency checks of this proposalГespecially by relating it to supergravity which low-energy limit of M-Theory. In this chapter $\Gamma$ we are going to introduce this model and establish some basic notions.

\subsection{The BFSS Conjecture}

$\mathbf{M}$ (atrix)-Theory $\Gamma$ as the authors of [BFSS] called their model is introduced to be the infinitemomentum frame description of M-Theory. The infinite-momentum frame is reached by singling out one "longitudinal" direction $x^{11}$ and then boosting the whole system so strongly that all longitudinal momenta appearing in a given physical situation are much larger than any other energy scale in the system. If we assume the $x^{11}$ direction to be compact with a radius $R_{11}$ the longitudinal momentum will be quantized as

$$
p_{11}=\frac{N}{R_{11}} .
$$

Uncompactified M-Theory is supposed to be recovered by taking $R_{11}$ and $N$ to infinity Kkeeping $p_{11}$ fixed. Since $N$ has to be an integer and is conserved in time $\Gamma$ we can think of it as the number of "partons" contributing to the physical situation at hand. They constitute finitely many particles that can only be exchanged but neither be created nor annihilated. In this framework $\Gamma$ the full machinery of quantum field theory appears to be unnecessary and can be replaced by the much simpler notions of quantum mechanics.

If the boost is actually infinite $\Gamma$ the relativistic dispersion relation

$$
M^{2}=2 p_{+} p_{-}-\vec{p}^{2}
$$

(we use vector notation for space-like directions perpendicular to the longitudinal one) simplifies dramatically after interpreting $p_{+}$as the "light-cone energy"):

$$
E=p_{+}=-\frac{R_{11} M^{2}}{2 N}+\frac{R_{11} \vec{p}^{2}}{2 N}
$$

Up to a constant $\Gamma$ we are left with a non-relativistic dispersion relation where $N \Gamma$ the total number of partons $\Gamma$ plays the rôle of the mass. The theory is not longer Lorentz invariant but we are left with Galilean invariance in the transverse directions. Thus $\Gamma$ we should be able to describe physics in the language of non-relativistic quantum mechanics.

Next $\Gamma$ we can use the dictionary from the previous chapter that relates elevendimensional objects of M-Theory compactified on a small circle to ten-dimensional objects 
of type IIA string theory: We have seen that the Kaluza-Klein modes in the compactified direction carry RR-one-form charge and are therefore D0-particles. We can identify the $x^{11}$-direction from above with the compact direction that relates M-Theory and IIA string theory $\Gamma$ and find that $N$ has to be interpreted as the D0 charge from a ten-dimensional perspective.

It is therefore natural to assume that the partons from above are nothing but the D0-particles when viewed from a ten-dimensional perspective. This is the conjecture put forward in [BFSS]: M-Theory in the infinite momentum frame is given by the dynamics of $N$ D0-particles in the $N \rightarrow \infty$ limit.

As we have explained in the previous chapter $\Gamma$ the low-energy effective dynamics of $N \mathrm{D} p$-branes is given by the supersymmetric $U(N)$ gauge theory of the open strings connecting the $\mathrm{D} p$-branes $\Gamma$ dimensionally reduced to the $(p+1)$-dimensional world volume. In the case of D0-particles $\Gamma$ the world volume is only $(0+1)$-dimensional. Thus $\Gamma$ all fields only depend on time. Alternatively $\Gamma$ we can say that all space derivatives $\partial_{i}$ for $i=1, \ldots, 9$ vanish. In the field-strength (all fields are $u(N)$ matrices)

$$
F_{\mu \nu}=\partial_{\mu} A_{\nu}-\partial_{\nu} A_{\nu}+\left[A_{\mu}, A_{\nu}\right]
$$

the only remaining derivatives are

$$
F_{0 i}=-F_{i 0}=\partial_{t} A_{i}+\left[A_{0}, A_{i}\right]
$$

and in the space-space components Tonly the commutator survives:

$$
F_{i j}=\left[A_{i}, A_{j}\right]
$$

It is common in the M(atrix)-Theory literature to rename the bosonic fields as $X^{i}=A_{i}$ and $A=A_{0}$. In these variables $\Gamma$ the M(atrix)-Theory Lagrangian reads

$$
\begin{aligned}
L & =\operatorname{Tr}\left(-\frac{1}{4} F^{\mu \nu} F_{\mu \nu}+\bar{\psi} D \psi\right) \\
& =\operatorname{Tr}\left(\frac{1}{2} \dot{X}^{i} \dot{X}^{i}+\frac{1}{4}\left[X^{i}, X^{j}\right]\left[X_{i}, X_{j}\right]-i \bar{\psi} \dot{\psi}+\bar{\psi} \gamma^{i}\left[X_{i}, \psi\right]\right)
\end{aligned}
$$

if we denote covariant derivatives by a dot as $\dot{X}^{i}=\partial_{t} X^{i}+\left[A, X^{i}\right]$. Note $\Gamma$ that this really is the Lagrangian and not the Lagrangian density $\Gamma$ as all spatial dependence of the fields is gone and we are left with a quantum mechanical rather than a field theoretic model.

It is possible to choose the Coulomb gauge $A=0$ but only to the price of requiring gauge invariance afterwards by imposing a Gauß law constraint

$$
\left[\dot{X}^{i}, X_{i}\right]-\frac{i}{2}[\bar{\psi}, \psi]=0
$$


In most parts of our discussion $\Gamma$ especially when doing quantum loop computations $\Gamma$ we will therefore not fix this gauge.

The model is invariant under supersymmetries given by two constant spinors $\epsilon$ and $\eta$ as

$$
\begin{aligned}
\delta X^{i} & =-2 \bar{\epsilon} \gamma^{i} \psi \\
\delta \psi & =\left(i \dot{X}_{i} \gamma^{i}+\gamma_{-}+\frac{1}{2}\left[X^{i}, X^{j}\right] \gamma_{i j}\right) \epsilon+\eta \\
\delta A & =-2 \bar{\epsilon} \psi,
\end{aligned}
$$

one acting linearly and one acting nonlinearly. This fits with the expectation from lowenergy M-Theory aka. supergravity which has 32 independent supersymmetries. As one can see $\Gamma$ the supersymmetry variation includes a gauge transformation and therefore the supersymmetry algebra only closes on gauge invariant states.

The sixteen linearly realized supersymmetries can be grouped into eight fermionic creation and eight annihilation operators as described for example in [dWHN]. They span a 256-dimensional fermionic Fock space whose representation content under the transversal $S O(9)$ is

\section{$44 \oplus \mathbf{8 4} \oplus \mathbf{1 2 8}$.}

Those are nothing but the representations of the graviton $g_{M N} \Gamma$ the graviphoton $A_{M N P}$ and the Rarita-Schwinger gravitino $\Psi_{M}$ unter the $S O(9)$ little group that make up the eleven-dimensional supergravity-multiplet.

Thus Tif there is exactly one massless state in the M(atrix)-Model $\Gamma$ the eight fermionic creation operators build the states of eleven-dimensional supergravity on top of it. Therefore $\Gamma$ to possibly recover supergravity from the $\mathrm{M}$ (atrix)-Model it is essential that there is exactly one massless state in the model that describes a marginal bound state of $N$ D0-particles from the stringy perspective. Already in [dWLN] it was shown that the model has a continuous spectrum for all energies $E \geq 0$ एbut there the question of zero energy states could not be resolved.

In [W] $\Gamma$ Witten gave an argument for the existence of exactly this one bound state using string dualities. In [SS] $\Gamma$ Sethi and Stern worked out the Witten index $\operatorname{tr}(-1)^{\# \text { fermions }}$ for the $N=2$ case and found it to be one strengthening the believe in the existence of a unique ground state. Also $[\mathbf{P R}] \Gamma[\mathbf{Y}] \Gamma[\mathbf{S m}] \Gamma[\mathbf{K N S}] \Gamma[\mathbf{F H}] \Gamma[\mathbf{H S}] \Gamma[\mathbf{M N S}]$ and $[\mathbf{K S}]$ were concerned with the difficult problem of identifying the unique ground state.

In all what we have said so farTwe thought of $N$ as being a special kind of regulator. Finite $N$ physics was meant as an approximation and the full eleven-dimensional theory should only be recovered in the limit $N \rightarrow \infty$. Especially $\int d=11$ Lorentz invariance is only expected in this limit as a boost in the $x^{11}$ direction changes $N$. But in spring $1997 \Gamma$ Susskind put forward "Another Conjecture about M(atrix)-Theory" [Su]: He proposed that the finite $N \mathrm{M}$ (atrix)-Model is an exact description of M-Theory compactified on a lightlike circle with $N$ units of momentum in the compact direction. This second conjecture 
was mainly based upon the observation that most consequences of the first conjecture presented above work equally well for finite $N$ and that already the finite $N$ gauge theory has a number of duality symmetries one believes are essential for a model to describe M-Theory.

One should be aware that a compactification on a light-like circle is at least on the verge of being unphysical: It nearly violates causality. Furthermore $\Gamma$ the notion of the radius $R_{11}$ of the light-like circle is not invariant under Lorentz transformations. Therefore $\Gamma$ it should finally drop out of any expressions for observable quantities.

Seiberg[Sei] and Sen[Se] presented a "derivation" of Susskind's finite $N$ conjecture using the relations (2.5) along the following lines: Compactification on a light-like circle means we are going to identify coordinates as

$$
\left(\begin{array}{c}
x^{11} \\
t
\end{array}\right) \sim\left(\begin{array}{c}
x^{11} \\
t
\end{array}\right)+\left(\begin{array}{c}
R_{11} / \sqrt{2} \\
-R_{11} / \sqrt{2}
\end{array}\right) .
$$

In a first step $\Gamma$ we are tilt the circle by a small amount $R_{s}$ to make it space-like:

$$
\left(\begin{array}{c}
x^{11} \\
t
\end{array}\right) \sim\left(\begin{array}{c}
x^{11} \\
t
\end{array}\right)+\left(\begin{array}{c}
\sqrt{R_{11}^{2} / 2+R_{s}^{2}} \\
-R_{11} / \sqrt{2}
\end{array}\right)
$$

In step two Twe boost the system with rapidity

$$
\beta=\frac{R_{11}}{\sqrt{R_{11}^{2}+2 R_{s}^{2}}}
$$

to obtain the "standard" compactification on the space-like circle

$$
\left(\begin{array}{c}
x^{11} \\
t
\end{array}\right) \sim\left(\begin{array}{c}
x^{11} \\
t
\end{array}\right)+\left(\begin{array}{c}
R_{s} \\
0
\end{array}\right)
$$

with small radius $R_{s}$. By definition $\Gamma$ M-Theory compactified on a small circle is type IIA string theory with $p_{-}=N / R_{s}$ D0-particles. During the boost $\Gamma$ the light-cone energy $p_{+}$ was rescaled by a factor $R_{s} / R$. In a third step $\Gamma$ we can undo this rescaling by passing to a "second" $\tilde{M}$-Theory with rescaled Planck length $\tilde{\ell}_{p}$ by demanding that

$$
\frac{R_{11}}{\ell_{p}^{2}} \sim p_{+} \sim \frac{R_{s}}{\tilde{\ell}_{p}^{2}}
$$

is fixed. Now $\Gamma$ we can use the dictionary $(2.5)$ to find the string theory:

$$
\tilde{\alpha}^{\prime}=\tilde{\ell}_{s}^{2}=\frac{\ell_{p} \sqrt{R_{s}}}{R_{11}^{3 / 2}}
$$

which goes to zero as we take $R_{s}$ to zero in the end to undo the tilt. This means that we can ignore higher curvature corrections to the string effective action. The exited string 
states become infinitely massive and decouple. Thus Tthe effective description of the strings stretching between the D0-particles is really given by a gauge theory and not by a BornInfeld theory or any other kind of higher derivative theory. Furthermore $\mathrm{Wwe}$ find

$$
\tilde{g}_{s}=\frac{R_{11}^{3 / 4} R_{s}^{3 / 4}}{\ell_{p}^{3 / 2}}
$$

which also vanishes in the $R_{s} \rightarrow 0$ limit. This means that the closed strings and therefore gravity decouples in this limit. We are left with a pure gauge theory.

Any transverse scales $R_{\perp}$ are not affected by the boost. Thus we should have

$$
\frac{R_{\perp}}{\ell_{p}}=\frac{\tilde{R}_{\perp}}{\tilde{\ell}_{p}}
$$

which implies

$$
\frac{\tilde{R}_{\perp}}{\tilde{\ell}_{s}}=\frac{R_{\perp} R_{11}^{1 / 4} R_{s}^{1 / 4}}{\ell_{p}^{3 / 2}} .
$$

This means that from the stringy perspective $\Gamma$ the transverse distances between the D0particles vanish and in the light-like limit $R_{s} \rightarrow 0$ the D0-particles coincide. Therefore $\Gamma$ we find the gauge group $U(N)$ to be unbroken $\Gamma$ as the distances between the particles are proportional to Higgs expectation values.

This concludes the argument that M-Theory compactified on a light-like circle is given by ten-dimensional supersymmetric $U(N)$ gauge theory dimensionally reduced to the world-line of $N$ coinciding particles.

\subsection{Do-Particles As Particles}

Let us inspect the M(atrix)-Model-Lagrangian (3.1) a bit closer. For a moment the fermionic fields to 0 . We see that it has the well known structure of kinetic energy minus potential energy

$$
L_{\text {boson }}=T-V, \quad T=\frac{1}{2} \operatorname{Tr} \dot{X}^{i} \dot{X}^{i}, \quad V=-\frac{1}{2} \operatorname{Tr}\left[X^{i}, X^{j}\right]^{2}
$$

We would like to guess low energy solutions to the equations of motion. As we have used the natural unit system $c=\hbar=\kappa=1 \Gamma$ low energy corresponds to $E \ll 1$. This implies that especially the potential energies very small and vanishes in the limit $E=0$. This equivalent to

$$
V=0 \Longleftrightarrow \forall i, j:\left[X^{i}, X^{j}\right]=0
$$


and we can — as in quantum mechanics — diagonalize all the matrices $X^{i}$ simultaneously:

$$
X^{i}(t)=\left(\begin{array}{lll}
\lambda_{1}^{i}(t) & & \\
& \ddots & \\
& & \lambda_{N}^{i}(t)
\end{array}\right)
$$

Plugging this ansatz into (3.1) Twe find a free equation of motion for the eigenvalues:

$$
\ddot{\lambda}_{a}^{i}=0 \quad \Rightarrow \quad \lambda_{1}^{i}(t)=b_{a}^{i}+v_{a}^{i} t
$$

with some constants of integration $b_{a}^{i}$ and $v_{a}^{i}$. An important observation of [BFSS] was that one can regroup the eigenvalues into vectors

$$
\vec{\lambda}_{a}=\left(\lambda_{a}^{1}, \ldots, \lambda_{a}^{9}\right)
$$

and interpret them as the coordinates of $N$ particles (namely the D0 branes) at time $t$. At the classical level $\Gamma$ we just have free $\Gamma$ straight line motions with impact parameters $\vec{b}_{a}$ and velocities $\vec{v}_{a}$.

The center of mass motion is encoded in the traces of the matrices:

$$
\lambda_{\mathrm{cm}}^{i}=\frac{1}{N} \operatorname{Tr} X^{i}
$$

If we decompose the gauge group as $U(N)=S U(N) \times U(1)$ in terms of the determinant and decompose the matrices as

$$
X^{i}=\tilde{X}^{i} \oplus \lambda^{i}
$$

we see that $\Gamma$ even without the diagonal ansatz $\Gamma$ the center of mass motion does not contribute to the commutators and therefore decouples. This is as we would have expected it for a system of particles without external fields. From now on trivial center of mass motion and take all matrices to be from $s u(N) \Gamma$ i.e. to be traceless.

If we did not know about the ten- or eleven-dimensional origin of this model we could use this manifold of low energy solutions (coordinatized by the eigenvalues of the matrices) to define the physical space-time of this configuration space. This a posteriori construction of space-time can be viewed from a more general perspective: The idea is to start with some auxiliary space-time of any dimension (here: the real line) and study some field theory on this space. Find the moduli space of vacuum configurations and interpret it as the configuration space of particles (or maybe other objects) moving in the true physical spacetime. If the field theory one started with is originating from a D-brane construction $\Gamma$ the term "D-geometry" has been coined by Douglas for spaces obtained in this way.

Generically 5 the space of classical vacua will be very different from the space of vacua of the full quantum theory that even might be completely out of reach. But for 
supersymmetric models $\Gamma$ the situation is often better and some properties of the classical moduli space will be preserved in the process of quantization.

The off-diagonal matrix entries will be shown in the next chapter to be massive with a mass proportional to the separation of the particles. They should be interpreted as the degrees of freedom of the strings stretching between the different D0-particles. They can be ignored for large separations (as compared to the string scale) as they will be frozen in their ground states. But for situations in which some D0-particles get close to each other the off-diagonal degrees of freedom become light and start interacting.

The diagonal matrices of generic low energy configurations commute as do usual coordinates. But in the close limit situations with off-diagonal matrix entries turned on $\Gamma$ this is no longer the case. This non-commutativity at short distances is believed to be a generic feature of quantum space-times.

\subsection{T-Duality In The M(atrix)-Model}

As we have explained in the introduction $\Gamma \mathrm{T}$-duality is a symmetry of string theories that relates compactifications on large circles to compactifications on large circles. If a spacetime with a D-brane is T-dualized the open string boundary conditions get exchanged and the $\mathrm{D} p$-brane is turned into a $\mathrm{D}(p+1)$ - or a $\mathrm{D}(p-1)$-brane.

This T-duality should also be visible from the M(atrix)-Model-perspective and we will present here the approach by Taylor[T1]. The idea is the compactification of one direction $\Gamma x^{1}$ say $\Gamma$ on a circle of radius $R$ is indistinguishable from an uncompactified situation in which each D0-particle has an infinite series of "mirror particles" at positions that differ by $2 \pi R \mathbb{Z} \vec{e}_{1}$ :

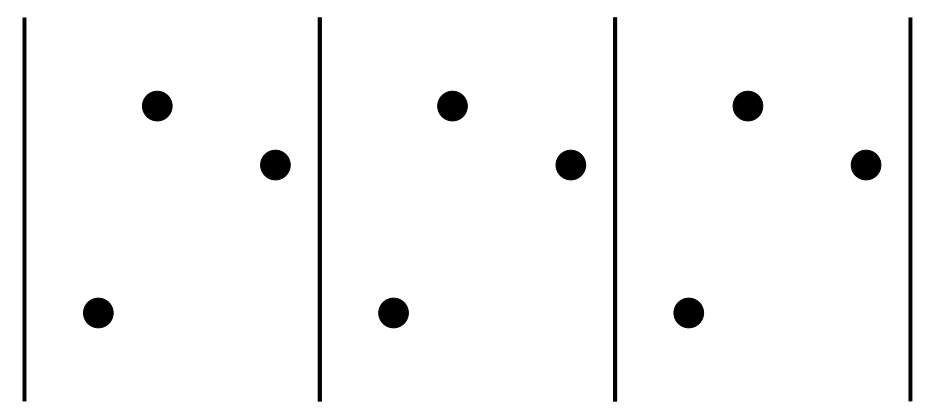

Fig. 3: Mirror particles and fundamental regions

Including all mirror particles we have an infinite number of D0-particles and therefore have to deal with infinite matrices. But those matrices have a very simple structure: If there are $N$ particles in one fundamental region $\Gamma$ we can take them to consist of $N \times N$ blocks. On the block diagonal $\Gamma$ the entries describe interactions within one fundamental 
region $\Gamma$ one block right of the diagonal $\Gamma$ the blocks describe interactions across one boundary of the fundamental region and so on. As the $N$ D0-particles are in the same positions in the non-compact directions $\Gamma$ the matrices for $i>0$ are of the form

$$
X^{i}=\left(\begin{array}{ccccc}
\ddots & \vdots & \vdots & \vdots & \\
\cdots & X^{i}(0) & X^{i}(1) & X^{i}(2) & \cdots \\
\cdots & X^{i}(-1) & X^{i}(0) & X^{i}(1) & \cdots \\
\cdots & X^{i}(-2) & X^{i}(-1) & X^{i}(0) & \cdots \\
& \vdots & \vdots & \vdots & \ddots
\end{array}\right)
$$

with $X^{i}(k) \in u(N)$ and the argument indicating the offset from the block diagonal. The positions for $i=1$ differ by $2 \pi R$ in the compact direction. Therefore $\Gamma$ we find there

$$
X^{1}=\left(\begin{array}{ccccc}
\ddots & \vdots & \vdots & \vdots & \\
\cdots & X^{1}(0) & X^{1}(1) & X^{1}(2) & \cdots \\
\cdots & X^{1}(-1) & X^{1}(0)+2 \pi R & X^{1}(1) & \cdots \\
\cdots & X^{1}(-2) & X^{1}(-1) & X^{1}(0)+4 \pi R & \cdots \\
& \vdots & \vdots & \vdots & \ddots
\end{array}\right)
$$

We can use a more compact notation if we introduce the shift matrix

$$
U=\left(\begin{array}{lllll} 
& 1 & & & \\
& & 1 & & \\
& & & 1 & \\
& & & & 1 \\
1 & & & &
\end{array}\right)
$$

(extended to infinite size) and the clock matrix

$$
T=\left(\begin{array}{ccccc}
\ddots & & & & \\
& 0 & & & \\
& 2 \pi R & & \\
& & 4 \pi R & \ddots
\end{array}\right)
$$

Then $\Gamma$ the infinite matrices can be written as

$$
X^{i}=\sum_{n} X^{i}(n) \otimes U^{n}+\delta^{i 1} \mathbb{1} \otimes T
$$

The effect of multiplying by $U$ is to shift $n \mapsto n+1$. Alternatively $\Gamma$ we can employ an inverse Fourier transform to write

$$
\tilde{X}^{i}(\sigma)=\sum_{n} X^{i}(n) e^{i n \sigma}+\delta^{i 1} \mathbb{1} \otimes T
$$


Then $U$ Tinterpreted as an operator $\Gamma$ can be written as $e^{i \sigma}$ whereas multiplication by $T$ can be represented by $2 \pi i R \frac{\partial}{\partial \sigma}$. We find a new operator

$$
\hat{X}^{i}(\sigma, t)=\tilde{X}^{i}(\sigma)+2 \pi i R \delta^{i 1} \frac{\partial}{\partial \sigma} .
$$

We find again a gauge theory with finite dimensional gauge group $u(N)$ but now in two dimensions $t$ and $\sigma . \hat{X}^{i}$ is the covariant derivative in the new $\sigma$ direction and the remaining $\hat{X}^{i}$ for $i=2, \ldots, 9$ are scalar fields in the adjoint representation of the gauge group.

This is nothing but the $(1+1)$-dimensional gauge theory that is the low energy effective action describing $N$ coincident D1-branes. Therefore $\Gamma$ we have just given a description of T-duality in the M(atrix)-Model language: It is possible to trade an "infinity" in the rank of the matrices for a new spacetime dimension.

\subsection{The M(atrix)-Model and Supermembranes}

The context of M-Theory and the M(atrix)-Theory conjecture were not the first time the $\mathrm{M}$ (atrix)-Model was discussed. It was first introduced in $[\mathbf{C H}] \Gamma[\mathbf{F}]$ and $[\mathbf{B R R}]$ as an example of a quantum mechanical system with $\mathcal{N}=16$ extended supersymmetry and also in the context of supermembranes $[\mathbf{B S T}][\mathbf{H o}][\mathbf{d} \mathbf{W H N}]$. As we have already mentioned in the introduction $\Gamma$ membranes are fundamental degrees of freedom in M-Theory. Here $\Gamma$ we will only sketch why they are described by the same M(atrix)-Model as the membrane interpretation does not play a major rôle in this thesis except for a short remark in chapter 6 . For a much longer review of the connection between supermembranes and M-Theory $\Gamma$ see $[\mathbf{N H}]$.

The Nambu-Goto action of the supermembrane is obtained by measuring the world volume and a Wess-Zumino-Witten term using the supervielbein

$$
E_{i}{ }^{\mu}=\partial_{i} X^{\mu}+\bar{\theta} \Gamma^{\mu} \partial_{i} \theta
$$

Here $\Gamma$ we assumed the supermembrane to be embedded in an eleven-dimensional superspace. The action reads

$$
\mathcal{L}=-\sqrt{-g(X, \theta)}-\epsilon^{i j k}\left(\frac{1}{2} \partial_{i} X^{\mu}\left(\partial_{j} X^{\nu}+\bar{\theta} \Gamma^{\nu} \partial_{j} \theta\right)+\frac{1}{6} \bar{\theta} \Gamma^{\mu} \partial_{i} \theta \bar{\theta} \Gamma^{\nu} \partial_{j} \theta\right) \bar{\theta} \Gamma_{\mu \nu} \partial_{k} \theta
$$

It was discovered in [Ho] that this action becomes polynomial when written in light-cone gauge

$$
\partial_{i} X^{+}=\delta_{i 0}
$$

NamelyГthe Hamiltonian density reads

$$
\mathcal{H}=\frac{\vec{P}^{2}+g}{2 P^{+}}-\epsilon^{r s} \partial_{r} X^{i} \bar{\theta} \Gamma^{-} \Gamma_{i} \partial_{s} \theta
$$


where $r$ and $s$ run over the remaining two world indices and $i$ is a nine-dimensional transversal index. The metric density is given by

$$
g=\operatorname{det}_{r s}\left(\partial_{r} \vec{X} \cdot \partial_{s} \vec{X}\right)=\left(\epsilon^{r s} \partial_{r} X^{i} \partial_{s} X^{j}\right)^{2} .
$$

Another important observation was $\Gamma$ that this $\Gamma$ since it gives "the density of area" is invariant under a residual symmetry of the membrane diffeomorphism invariance. Namely invariant under the action of area preserving diffeomorphisms (APDs). Those are locally generated by vector fields that are curls of functions ("the potentials")

$$
\xi^{r}=\epsilon^{r s} \partial_{s} \xi
$$

The APDs form a Lie-group $\Gamma$ therefore their generators form a Lie-algebra with the bracket being given by the usual commutator of vector fields. This Lie-bracket can be translated into a bracket of functions by identification of the functions with the potential of the generator of an APDГvis.

$$
\{A, B\}(\sigma):=\epsilon^{r s} \partial_{r} A(\sigma) \partial_{s} B(\sigma)
$$

We can use this bracket to rewrite (3.2) as

$$
\mathcal{H}=\frac{1}{2 P^{+}}\left(\vec{P}^{2}+\left\{X^{i}, X^{J}\right\}^{2}\right)-\bar{\theta} \Gamma^{-} \Gamma_{i}\left\{X^{i}, \theta\right\} .
$$

But this is nothing but the one-dimensional super Yang-Mills theory where the gauge group is given by the infinite-dimensional group of APDs. It is convenient to decompose functions of the membrane into a complete orthonormal set of functions as

$$
X^{i}(\sigma)=\sum_{A} X_{A}^{i} Y_{A}(\sigma)
$$

In terms of those $\Gamma$ the structure constants of the Lie algebra can be obtained by

$$
f_{A B}{ }^{C}=\int d^{2} \sigma\left\{Y_{A}, Y_{B}\right\} Y_{C}
$$

It is a deep result that these structure constants for all possible membrane topologies can be obtained as a $N \rightarrow \infty$ limit of $S U(N)$ structure constants in a special basis[BMS]:

$$
\lim _{N \rightarrow \infty} f_{A B}^{C}(S U(N))=f_{A B}^{C}(\mathrm{APD})
$$

We will indicate how this limiting procedure works only for the simplest case of toroidal membranes. In this case $\Gamma$ we can decompose the functions in terms of their Fourier modes as

$$
Y_{\vec{m}}(\vec{\sigma})=\frac{1}{\sqrt{4} \pi} e^{i \vec{m} \cdot \vec{\sigma}}
$$


The APD structure constants follow from

$$
\left\{Y_{\vec{m}}, Y_{\vec{n}}\right\}=-4 \pi^{2}(\vec{m} \times \vec{n}) Y_{\vec{m}+\vec{n}}
$$

On the other hand $\Gamma$ we can use 't Hooft's clock and shift matrices

$$
V=\left(\begin{array}{cccc}
1 & & & \\
& \omega & & \\
& & \ddots & \\
& & & \omega^{N-1}
\end{array}\right), \quad U=\left(\begin{array}{cccc}
0 & 1 & & \\
& \ddots & \ddots & \\
& & \ddots & 1 \\
1 & & & 0
\end{array}\right)
$$

that generate $S U(N)$ if $\omega$ is an $N^{\text {th }}$ root of unity $e^{2 \pi i k / N}$. They commute up to a phase factor

$$
U V=\omega V U
$$

and the commutator of any $S U(N)$ matrices can be written in terms of

$$
\left[U^{m_{1}} V^{m_{2}}, U^{n_{1}} V^{n_{2}}\right]=\left(\omega^{m_{2} n_{1}}-\omega^{m_{1} n_{2}}\right) U^{m_{1}+n_{1}} V^{m_{2}+n_{2}}
$$

If we now take $N$ to infinity keeping $\vec{m}$ and $\vec{n}$ fixed $\Gamma$ this approaches

$$
\lim _{N \rightarrow \infty}\left[U^{m_{1}} V^{m_{2}}, U^{n_{1}} V^{n_{2}}\right]=\frac{2 \pi i k}{N}(\vec{m} \times \vec{n}) U^{m_{1}+n_{1}} V^{m_{2}+n_{2}} .
$$

Obviously this is just (3.3) $\Gamma$ the algebra of APDs on a torus.

This is just the toroidal case of the general fact that the action of supermembranes in eleven dimensions is the limit $N \rightarrow \infty$ of $S U(N)$ super-Yang-Mills quantum mechanics on the real line.

In the early days of supermembranes $\Gamma$ it was thought that membranes in eleven dimensions could be treated just like strings in ten but [dWLN] showed that the spectrum is continuous. Therefore $\Gamma$ there is no clear separation of particle masses in integer multiples of the membrane tension $1 / \ell_{p}$. Nowadays $\Gamma$ this continuous spectrum is viewed as an advantage as the membrane action we presented above is not the action of just one membrane but of several ones $\Gamma$ the membrane theory is automatically a second quantized theory: Since the potential energy is given by the area of the membrane additional infinitesimal tubes do not cost any energy. Thus $\Gamma$ one can use such tubes to connect separate membranes without changing the physical situation but turn several membranes into one that is again described by the above action.

In the M(atrix)-Model-language $\Gamma$ such a configuration can be described by block diagonal matrices. Each block describing one membrane. They interact in terms of the off-block-diagonal entries.

The reverse process can also be imagined: One membrane pinches off and "decays" into two parts that are only joined by an infinitesimal tube. Therefore $\Gamma$ it is not sensible to treat only single membranesTone always has to take into account multi-membrane states. 


\section{The M(atrix)-Model at One-Loop Order}

In this chapter we will present the basic setting of M(atrix)-Model scattering calculations. We will study in some detail the calculation that first hinted towards the possibility of studying processes in gravity from a gauge theory perspective. Then we proceed to calculate polarization dependant effects in order to demonstrate that we are really testing supergravity and not just some field theory in eleven dimensions. In the course of this study $\Gamma$ we will present different methods to obtain these results $\Gamma$ each suited best for its specific purpose. Especially 5 supergravity calculations can be quite lengthy if approached too naïvely but we will present more elegant ways to extract results in special cases.

\subsection{The Born-Oppenheimer Effective Potential}

In this section $\Gamma$ we are going to study leading order quantum corrections to the classical solutions of the M(atrix)-Model eequations of motion. In the simple free particles solution we have presented in the last chapter Tall off-diagonal matrix degrees of freedom were turned off and did not contribute. In a quantum theory $\Gamma$ the Heisenberg uncertainty principle does not allow such a turning off of degrees of freedom. We have to expect quantum fluctuations. Those fluctuations $\Gamma$ on the other hand $\Gamma$ will influence the dynamics of the diagonal matrix elements. This influence can be summarized by an effective potential on those "classical" degrees of freedom. After that we will compare the force described by this effective potential to the gravitational force between gravitons in eleven-dimensional supergravity. We will study in detail the leading order polarization independent potential and the static spin-spin interaction. The agreement we will find fits well into the connection between the M(atrix)-Model and supergravity via the conjectured M-Theory.

This approximation procedure should be compared to the Born-Oppenheimer approximation used in calculations of molecular physics: There Tone first fixes the positions of the nuclei and employs a quantum mechanical calculation to find the energy spectra of the electrons in the electric field of the nuclei. Then Tone interprets the ground state energy of the electrons $\Gamma$ viewed as a function of the positions of the nuclei $\Gamma a s$ an effective potential for the nuclei and solves for their classical trajectories in that potential. In both scenarios $\Gamma$ there is a separation into slow degrees of freedom that can be treated classically and fast $\Gamma$ fluctuating degrees of freedom that can be integrated out and via their interaction with the slow degrees of freedom leave an effective potential as their sole effect.

To get an idea of what we are going to expect $\Gamma$ let us perturb a diagonal matrix by some small off-diagonal quantity $\mu$ at position $(a, b)$ :

$$
X^{i}=\left(\begin{array}{ccccc}
\lambda_{1}^{i} & & & & \\
& \cdot & & \mu & \\
& & \cdot & & \\
& \bar{\mu} & & \cdot & \\
& & & \lambda_{N}^{i}
\end{array}\right)
$$


Plugging this ansatz into the bosonic part of the M(atrix)-Model-Lagrangian we find the terms containing $\mu$ to be

$$
L_{\mu}=\frac{1}{2} \dot{\bar{\mu}} \dot{\mu}-\frac{1}{2}\left\|\vec{\lambda}_{b}-\vec{\lambda}_{a}\right\|^{2} \bar{\mu} \mu
$$

But this is nothing but a harmonic oscillator with frequency $\omega=\left\|\vec{\lambda}_{b}-\vec{\lambda}_{a}\right\|$. We can assume $\omega$ to be constant for the fast oscillations of $\mu$ that are on the timescale of $1 / \omega$ which is very short for macroscopic separations $\left\|\vec{\lambda}_{b}-\vec{\lambda}_{a}\right\|$ of the particles described by the diagonal matrix elements. The quantization of this harmonic oscillator is basic textbook knowledge and we find the energy spectrum to be evenly spaced with level-spacing $\omega$. For macroscopic separations $\Gamma$ the oscillator will be frozen into its ground state of energy $\omega / 2$. Thus we are led to an effective potential for the diagonal matrix elements of the form

$$
V_{\mathrm{eff}}\left(\vec{\lambda}_{a}\right)=\frac{1}{2}\left\|\vec{\lambda}_{b}-\vec{\lambda}_{a}\right\|
$$

This potential fits well into the interpretation of the particles being D0-branes with strings stretching between them: The string between particle $a$ and $b$ has a length $\omega$ and therefore $\Gamma$ according to the stringy version of Hook's law We are led to identify the off-diagonal degree of freedom $\mu$ at position $(a, b)$ with the string degrees of freedom stretching between D0-branes $a$ and $b$.

On the other hand $\Gamma$ this potential seems to be disastrous for the asymptotic D0particle interpretation (remember that from the M-perspective the D0-branes are gravitons with momentum in the compact $\mathrm{M}$ direction): The potential increases for large separations and is therefore confining. There are no scattering states anymore $\Gamma$ all states are bound! But here $\mathrm{Tas}$ so oftenГsupersymmetry comes for a rescue: There are not only fluctuations on the bosonic off-diagonal fields $\Gamma$ also the fermionic matrices fluctuate and their effect is to turn the harmonic oscillator into a supersymmetric harmonic oscillator

$$
L=\frac{1}{2} \dot{\mu}^{2}-\frac{1}{2}\left\|\vec{\lambda}_{b}-\vec{\lambda}_{a}\right\|^{2} \mu^{2}+\frac{i}{2}(\bar{\theta} \dot{\theta}-\dot{\bar{\theta}} \theta)-2\left\|\vec{\lambda}_{b}-\vec{\lambda}_{a}\right\| \bar{\theta} \theta
$$

This supersymmetric extension is known to have the same energy level-spacing $\Gamma$ but as the fermions contribute with opposite sign in the full supersymmetric model $\Gamma$ the vacuum fluctuations do not contribute to a static potential that would correspond to a force between the D0-particles rendering the classical state unstable. This is in agreement with the BPS no-static-force property that we expect from the stringy picture of the situation.

To compute the full $\Gamma$ velocity dependent effective action to leading order quantitatively we will have to perform a more careful calculation. This is what we will do next. We will give a detailed presentation also to introduce some tools that we will need later on namely the background field method and the heat-kernel. 


\subsection{The Leading Polarization Independent Effective Potential}

The calculation we are going to describe here was first done by Douglas KKabat $\Gamma$ Pouliot and Shenker[DKPS] in the boundary state formalism of string theory give a description that is more intrinsic to the $\mathrm{M}$ (atrix)-Model and follows the lines of [BB].

The determination of quantum fluctuations around a classical solution is most easily done in the background field formalism. There one splits the dynamical fields into some classical part and the fluctuating quantum part as $\Phi=f+\phi$ and then expands the action in the quantum field:

$$
\mathcal{S}[\Phi]=\mathcal{S}[f]+\frac{\delta \mathcal{S}}{\delta \Phi(x)}[f] \phi(x)+\frac{1}{2} \phi(y) \frac{\delta^{2} \mathcal{S}}{\Phi(y) \delta \Phi(x)}[f] \phi(x)+O\left(\phi^{3}\right)
$$

The first term does not depend on $\phi$ and therefore only contributes a constant to the action that can be discarded further on. The second term vanishes if $f$ obeys the classical equations of motion $\delta \mathcal{S} / \delta \Phi=0$. The third term is the background dependent kinetic term $\phi \mathcal{O} \phi$ for the quantum field that determines the propagator $\frac{1}{\mathcal{O}}$. For a one-loop calculation $\Gamma$ this is all one needs $\mathrm{Tas}$ in a loop of the quantum field all vertices have exactly two quantum legs while all the other legs are external; vertices with more quantum legs would occur only at intersections of loops in higher loop diagrams. The external legs are represented by the background.

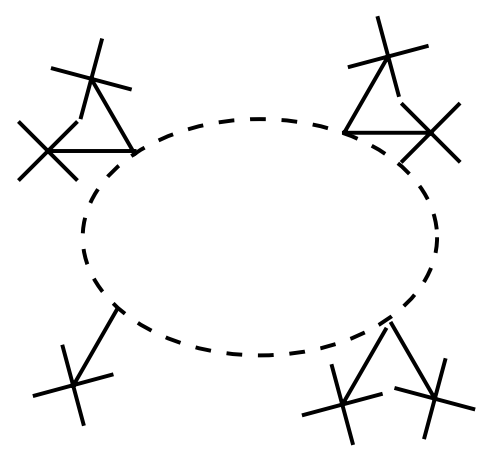

Fig. 4: One loop in the background field formalism

The one-loop effective action is given by

$$
\Gamma^{(1)}=\frac{1}{2} \operatorname{Tr} \log \mathcal{O}
$$

and can be conveniently calculated using an auxiliary function (it can be shown that this is really a function and not a distribution) called the heat-kernel $h(x, y, \sigma)$ [Sch]. It depends on two space-time points and an additional positive variable and is defined by the partial differential equation

$$
\mathcal{O}_{x} h=-\frac{\partial}{\partial \sigma} h
$$


together with the boundary condition $\lim _{\sigma \rightarrow 0} h(x, y, \sigma)=\delta(x, y)$. Note that symbolically we can write the heat-kernel as

$$
h(x, y, \sigma)=e^{-\sigma \mathcal{O}} \delta(x, y) .
$$

First $\Gamma$ we express the propagator in terms of $h$ :

$$
G(x, y)=\frac{1}{\mathcal{O}}=\int_{0}^{\infty} d \sigma h(x, y, \sigma)
$$

Indeed $\Gamma$ acting with $\mathcal{O}$ on $G(x, y)$ and using the defining PDE $\Gamma$ we obtain an integral over a total derivative with only the boundary at $\sigma=0$ contributing the desired delta function.

Next $\Gamma$ we ask how the effective action changes under variations of the operator $\mathcal{O}$. For example we could vary a parameter of the background:

$$
\begin{aligned}
\delta \Gamma^{(1)} & =\frac{1}{2} \delta \operatorname{Tr} \log \mathcal{O} \\
& =\frac{1}{2} \operatorname{Tr}\left(\frac{1}{\mathcal{O}} \delta \mathcal{O}\right) \\
& =\frac{1}{2} \operatorname{Tr} \int_{0}^{\infty} d \sigma \delta \mathcal{O} e^{-\sigma \mathcal{O}} \delta(x, y) \\
& =-\frac{1}{2} \operatorname{Tr} \int_{0}^{\infty} \frac{d \sigma}{\sigma} \delta h(x, y, \sigma)
\end{aligned}
$$

After integrating over the variation $\Gamma$ we end up with an expression for the effective action in terms of the heat-kernel:

$$
\Gamma^{(1)}=-\frac{1}{2} \operatorname{Tr} \int_{0}^{\infty} \frac{d \sigma}{\sigma} h(x, y, \sigma)
$$

Here $\Gamma$ the trace is understood as a functional trace not only over possible color indices (in general $\Gamma$ the heat-kernel carries two indices that run over all contributing fields; we will suppress these indices here) but also includes setting $y$ to $x$ and integrating over space-time.

In most applications of the heat-kernel $\Gamma$ one has to use approximations of $h$ as a power series in $\sigma$ Tbut in the case at hand this will not be necessary as for the operator $\mathcal{O}$ that appears in the M(atrix)-Model $\Gamma$ the heat-kernel is known exactly.

The planar one-loop gauge theory vacuum bubble in 't Hooft ribbon notation has two boundaries so it represents two particle scattering as we will see later on. Let us therefore anticipate that the one-loop calculation can be done with $N=2$ without loss of generality.

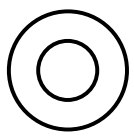

Fig. 5: One loop ribbon diagram 
Following [BB] we perform the background quantum splitting for the M(atrix)-Model and use the free particle solution to the equations of motion as the background field. The background is

$$
B^{i}=\frac{i}{2}\left(\begin{array}{cc}
r^{i} & 0 \\
0 & -r^{i}
\end{array}\right)
$$

with $\vec{r}=\vec{b}+\vec{v} t$. By a shift of $t$ we can assume the impact parameter $\vec{b}$ to be orthogonal to the velocity $\vec{v}$. The quantum fields are denoted by $\vec{Y}$ and can be decomposed with respect to a Pauli matrix basis of $s u(2)$ :

$$
\vec{Y}=\vec{Y}_{a} \sigma_{a}
$$

Plugging this into the bosonic Lagrangian $\Gamma$ we find for the quantum fields

$$
\begin{aligned}
L_{Y}=\frac{1}{2} & Y_{1}^{i}\left(\partial_{t}^{2}-r^{2}\right) Y_{1}^{i}+\frac{1}{2} Y_{2}^{i}\left(\partial_{t}^{2}-r^{2}\right) Y_{2}^{i}+\frac{1}{2} Y_{3}^{i} \partial_{t}^{2} Y_{3}^{i} \\
& -\epsilon^{a 3 d} \epsilon^{c b d} B^{i} Y_{a}^{j} Y_{b}^{i} Y_{c}^{j}-\frac{1}{4} \epsilon^{a b e} \epsilon^{c d e} Y_{a}^{i} Y_{b}^{j} Y_{c}^{i} Y_{d}^{j}
\end{aligned}
$$

In the previous chapter $\Gamma$ we have gauged away the only remaining gauge field $A=A_{0}$ that renders the time derivative covariant. This gauge is accompanied by the Gauss law as a constraint. Therefore it is not very convenient. The background field method offers a possibility to fix a gauge for the quantum fields while maintaining manifest background gauge invariance. This is done by imposing the condition that the divergence of the gauge field with respect to the background vanishes:

$$
\bar{D}^{\mu} A_{\mu}=\partial^{\mu} A_{\mu}+\left[B^{\mu}, A_{\mu}\right]=0
$$

In our case this just amounts to adding a term $\left(\partial_{t} A+\left[Y^{i}, B^{i}\right]\right)^{2}$ to the Lagrangian. Furthermore $\Gamma$ we have to use covariant time derivatives $\partial_{t}+[B, \cdot]$ for all the fields. We find the terms in the bosonic Lagrangian containing the gauge field to be

$$
\begin{gathered}
L_{A}=\frac{1}{2} A_{1}\left(\partial_{t}^{2}-r^{2}\right) A_{1}+\frac{1}{2} A_{2}\left(\partial_{t}^{2}-r^{2}\right) A_{2}+\frac{1}{2} A_{3} \partial_{t}^{2} A_{3}+2 \epsilon^{a b 3} v^{i} A_{a} Y_{b}^{i} \\
-\epsilon^{a 3 d} \epsilon^{b c d} B^{i} A_{a} A_{b} Y_{c}^{i}-\frac{1}{2} \epsilon^{a b e} \epsilon^{c d e} A_{a} Y_{b}^{i} A_{c} Y_{d}^{i}+\epsilon^{a b c} \partial_{t} Y_{a}^{i} A_{b} A_{c}^{i} .
\end{gathered}
$$

The last term in the first line is bilinear in the gauge field and the component of $\vec{Y}$ that is parallel to $\vec{v}$. These fields therefore mix and we have to diagonalize the corresponding mass matrix

$$
-\left(Y_{\|}, A\right)\left(\begin{array}{cc}
r^{2} & -2 v \\
-2 v & r^{2}
\end{array}\right)\left(\begin{array}{c}
Y_{\|} \\
A
\end{array}\right) .
$$

The eigenvalues are easily found to be $r^{2} \pm 2 v$. Thus we find the bosonic mass spectrum to consist of ten massless bosons $\left(A_{3}\right.$ and the nine components of $\left.\vec{Y}_{3}\right) \Gamma$ sixteen bosons of mass $m^{2}=r^{2}$ (the components of $\vec{Y}_{1 / 2}$ that are perpendicular to $\vec{v}$ ) and two plus two bosons of mass $m^{2}=r^{2} \pm 2 v$ (the appropriate linear combinations of $A_{1 / 2}$ and $Y_{\| 1 / 2}$ ). 
By varying the gauge fixing term with respect to a gauge transformation $\Gamma$ we find the Lagrangian for the Fadeev-Popov ghosts:

$$
\begin{gathered}
L_{C}=\bar{C}_{1}\left(-\partial_{t}^{2}+r^{2}\right) C_{1}+\bar{C}_{2}\left(-\partial_{t}+r^{2}\right) C_{2}+\bar{C}_{3} \partial_{t}^{2} C_{3} \\
+\epsilon^{a b c} \partial_{t} \bar{C}_{a} C_{b} A_{c}-\epsilon^{a 3 d} \epsilon^{c b d} B^{i} \bar{C}_{a} C_{b} Y_{c}^{i}
\end{gathered}
$$

The ghost fields are complex; in terms of real fields we have again four fields with $m^{2}=r^{2}$ and two massless ghost fields.

Writing $\psi_{ \pm}=\left(\psi_{1} \pm i \psi_{2}\right) / \sqrt{2} \Gamma$ the component Lagrangian for the fermions reads

$$
\begin{aligned}
L_{\psi}=\psi_{+} & \left(\partial_{t}-\beta\right) \psi_{-}+\frac{1}{2} \psi_{3} \partial_{t} \psi_{3} \\
& +\frac{1}{\sqrt{2}} \psi_{+}\left(Y_{1}-i Y_{2}\right) \psi_{3}+\frac{1}{\sqrt{2}} \psi_{3}\left(Y_{1}+i Y_{2}\right) \psi_{-} \\
& -i \frac{1}{\sqrt{2}}\left(A_{1}-i A_{2}\right) \psi_{+} i \psi_{3}+\frac{1}{\sqrt{2}}\left(A_{1}+i A_{2}\right) \psi_{3} \psi_{-} \\
& -\psi_{+} Y_{3} \psi_{-}+i A_{3} \psi_{+} \psi_{-} .
\end{aligned}
$$

In order to find the effective action for the fermions $\Gamma$ we employ the "doubling trick" that amounts to noting that for an operator $D$ (in our case $D=\partial_{t}-\beta$ ) we have

$$
\log D D^{\dagger}=\log D+\log D^{\dagger}=2 \log D
$$

as long as there are no subtleties due to fermionic zero modes in chiral models. For our calculation $\Gamma$ this yields

$$
p p^{\dagger}=\left(\partial_{t}-\beta\right)\left(-\partial_{t}-\beta\right)=-\partial_{t}^{2}-\left[\partial_{t}, \beta\right]+\beta \beta=-\partial_{t}^{2}-\psi+r^{2}
$$

Again $\Gamma$ we could diagonalize the mass matrix but we can also note that for each spatial $\gamma$-matrix $\Gamma$ half of the eigenvalues have to be 1 and the other ones have to be -1 since $\gamma^{i}$ is traceless and squares to the unit matrix. So half of the sixteen components of both $\psi_{ \pm}$ have $m^{2}=r^{2}-v$ and the other ones have $m^{2}=r^{2}+v$. The sixteen components of $\psi_{3}$ are massless.

Collecting the masses of all fields and taking care of the anti-commutativity of fermions and ghosts with a negative sign and a factor of $\frac{1}{2}$ for the fermions to compensate for the doubling trick $\Gamma$ we find the following table:

\begin{tabular}{|c|c|c|}
\hline \# real components & $m^{2}$ & weight factor \\
\hline 16 & $r^{2}$ & 1 \\
2 & $r^{2}-2 v$ & 1 \\
2 & $r^{2}+2 v$ & 1 \\
10 & 0 & 1 \\
4 & $r^{2}$ & -1 \\
2 & 0 & -1 \\
16 & $r^{2}-v$ & $-1 / 2$ \\
16 & $r^{2}+v$ & $-1 / 2$ \\
16 & 0 & $-1 / 2$ \\
\hline
\end{tabular}


In total $\Gamma$ the various massless fields cancel each other in the effective action $\Gamma$ which is what one should expect in a supersymmetric theory. All the remaining fields are massive. Thus we do not expect any infrared problems in the calculation. As "space-time" is only onedimensional $\Gamma$ there are no ultraviolet problems $\Gamma$ either $\Gamma$ and the whole calculation of the one-loop effective action is finite. One might wonder how $\Gamma$ in this setting $\Gamma$ field theoretic divergencies could occur at all $\Gamma$ since the system is quantum mechanical and has only a finite number of degrees of freedom. But this reasoning is not correct as by calculating scattering amplitudes we are asking a field theoretical question and thus we would have to face divergences in a generic system.

All the background dependent kinetic terms we have found contain derivative Conly terms constant with or quadratic in time. Therefore it sufficient to know that the heat-kernel of a field of mass $v t \Gamma$ say $\Gamma$

$$
e^{-\sigma\left(-\partial_{t}^{2}+v^{2} t^{2}\right)}
$$

is given by the propagator of a harmonic oscillator

$$
e^{-i \tau\left(P^{2}+v^{2} Q^{2}\right)}
$$

if we identify $\sigma$ with $i \tau \Gamma \partial_{t}$ with $i P$ and $t$ with $Q$ (note that the canonical commutation relations are fulfilled after this identification!). The exact propagator of the harmonic oscillator is well known (see for example [Fey]) and we find the heat-kernel for a field of mass $v t$ to be

$$
h\left(t, t^{\prime}, \sigma\right)=\sqrt{\frac{v}{2 \pi \sinh (2 \sigma v)}} \exp \left(-v t_{-}^{2} \operatorname{coth}(\sigma v)-v t_{+}^{2} \tanh (\sigma v)\right)
$$

where we introduced $t_{ \pm}=\left(t \pm t^{\prime}\right) / 2$. If there is a constant term of the form $b^{2}$ in the expression for $m^{2} \Gamma$ it contributes a factor of

$$
\exp \left(-\sigma b^{2}\right)
$$

to the heat-kernel since it commutes with $\partial_{t}$ and therefore no Campbell-Baker-Hausdorfflike formulas have to be applied. Putting everything together and taking the trace over all the fields we find

$$
\begin{aligned}
\Gamma^{(1)} & =-\frac{1}{2} \int_{-\infty}^{\infty} d t \int_{0}^{\infty} \frac{d \sigma}{\sigma} e^{-\sigma b^{2}}(12+4 \cosh (\sigma 2 v)-16 \cosh (\sigma v)) h(t, t, \sigma) \\
& =-\frac{1}{2} \int_{-\infty}^{\infty} d t \int_{0}^{\infty} d \sigma \sqrt{\frac{1}{\pi \sigma}} \sigma^{3} e^{-\sigma r^{2}} v^{4}+O\left(v^{6}\right) \\
& =\int_{-\infty}^{\infty} d t\left(-\frac{15}{16} \frac{v^{4}}{r^{7}}\right)+O\left(v^{6}\right) .
\end{aligned}
$$

Thus we have found an effective potential proportional to the fourth power of the relative velocity. NaïvelyГon dimensional grounds Tone might have expected also terms proportional 
to $r$ or to $v^{2} / r^{3}$ (odd powers of $v$ have to be absent since they change sign under time reversal). Those terms of lower order in the velocity are absent due to the high amount of supersymmetry. The first one is just the static force we found above in the purely bosonic reasoning while the second is typically present in situations that preserve only one quarter of supersymmetry from the ten-dimensional perspective $\Gamma$ like a D4-D0 bound stateГ and not half of the supersymmetry as in the purely D0 scenario we consider here[DKPS].

\subsection{Newton's Law and Coulomb's Law in the Light-Cone Frame}

If the strongest $\mathrm{M}$ (atrix)-Theory conjecture is true and the finite $N \mathrm{M}$ (atrix)-Model is a description of M-Theory the effective action we have worked out above should correspond to some potential in eleven-dimensional supergravity which by definition is the low-energy limit of M-Theory. Instead of performing a full supergravity calculation at this point (that we would have to redo in a later chapter where we will study three-particle scattering) $\Gamma$ we will consider a related scenario in simple electrodynamics that is much simpler to analyze but bears the same features as the gravitational calculation.

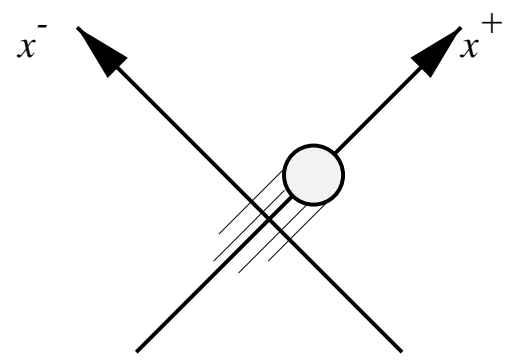

Fig. 6: A particle moving in light-cone coordinates

Let us consider a massless charged particle that travels at the speed of light in $x^{1}$ direction in $d$-dimensional space-time $\Gamma$ i.e. its world-line coincides with the $x^{+}=\left(x^{0}+x^{1}\right) / 2$ direction. It produces a current

$$
\mathbf{j} \propto j \delta\left(x^{-}\right) \delta\left(x_{\perp}\right) \mathbf{e}_{-}
$$

where we have denoted the light-cone coordinates by $x^{ \pm}$and the $d-2$ transversal coordinates collectively by $x_{\perp}$. Here $\mathbf{e}_{-}$is the one-form dual to $\frac{\partial}{\partial x^{+}}$. To find the electro-magnetic field of the particle we solve Maxwell's equation

$$
j^{\mu}=\square A^{\mu}=\left(-2 \partial_{-} \partial_{+}+\Delta_{\perp}\right) A^{\mu} .
$$

The first term vanishes since the situation is invariant under translations in the $x^{+}$direction. We find the only non-vanishing component of the potential to be

$$
A_{-}(x) \propto \delta\left(x_{-}\right) \frac{1}{\left\|x_{\perp}\right\|^{d-4}}
$$


where $\left\|x_{\perp}\right\|^{0}$ is understood as $\log \left\|x_{\perp}\right\|$. We find that all components of the field strength except for $F_{i-}$ and $F_{-i}$ vanish.

Next we shoot a second test particle through this electro-magnetic field to find the force that is acting on it. We assume the test particle to be massless $\Gamma$ too $\Gamma$ thus it also travels at the speed of light. We denote the components of its velocity in the transversal directions by $v_{\perp}$ and find its $d$-dimensional velocity to be

$$
\mathbf{u}=\left(1, \sqrt{1-v_{\perp}^{2}}, v_{\perp}\right)
$$

in $\left(x^{0}, x^{1}, \ldots, x^{d-1}\right)$ coordinates. The acceleration the test particle feels due to the electromagnetic field is given by

$$
\frac{d u^{i}}{d \tau}=u_{\mu} F^{\mu i}=u_{+} F^{+i}
$$

But

$$
u_{+} F^{+i}=u^{-} F_{-i} \propto \frac{1-\sqrt{1-v_{\perp}^{2}}}{2} \frac{\delta\left(x^{-}\right) x^{i}}{\left\|x_{\perp}\right\|^{d-6}} \propto \frac{v_{\perp}^{2}}{4} \delta\left(x^{-}\right) \partial^{i} \frac{1}{\left\|x_{\perp}\right\|^{d-4}}+O\left(v_{\perp}^{6}\right) .
$$

Thus $\Gamma$ to the leading order $\Gamma$ we have found a potential

$$
V \propto \delta\left(x^{-}\right) \frac{v_{\perp}^{2}}{\left\|x_{\perp}\right\|^{d-4}}
$$

that is proportional to the square of the transversal velocity one might have expected from applying Coulomb's law naïvely. Especially of the two particles is parallel there is no force at all and the configuration is stable! One could proceed adding charged particles flying in that direction and still have a solution to the equations of motion. Thus we have found a superimposable solution to the $\Gamma$ in general $\Gamma$ nonlinear system of coupled equations of motion and field equations. The physical reason for this linearization is that $\Gamma$ in the case of particles traveling at the speed of light $\Gamma$ the static Coulomb force and the velocity dependent Lorentz force cancel.

In fact one can express this cancellation in more elaborate terms: In a supersymmetrized version of electro-dynamics $\Gamma$ this field configuration $\Gamma$ with all additional fermion fields consistently set to zero is a BPS configuration that preserves half of the supersymmetry. The linearization is just the familiar superposition of BPS configurations that is also well known from extremal Reissner-Nordström black holes. Another property of the potential is the fact that the distance dependence is by two powers weaker than the $1 / x^{d-2}$ dependence of the usual Coulomb law.

Because of the special form of the field-strength $F_{i-} \Gamma$ there is no way to contract more than two field strength tensors with at most two free indices even with the help of an $\epsilon$ tensor since there is no tensor with two upper $(-)$-indices. Therefore $\Gamma a n y$ corrections to 
this solution in higher derivative-versions of electro-dynamics vanishes and we can conclude that we have found an exact solution even for higher derivative-corrected theories $\Gamma$ that for example might arise as effective theories from quantization of this theory.

For gravity 5 there is a solution to the Einstein equations that corresponds to (4.3). It describes a "massless black hole" that is boosted to the speed of light and is known as pp-wave or as Aichelburg-Sexl metric [AS] $\Gamma$

$$
d s^{2}=d s_{\text {flat }}^{2}+h_{--} d x^{-} d x^{-}=\eta_{\mu \nu} d x^{\mu} d x^{\nu}+\alpha \frac{\delta\left(x^{-}\right)}{\left\|x_{\perp}\right\|^{d-4}} d x^{-} d x^{-},
$$

with arbitrary $\alpha$. This solution $\Gamma$ dimensionally reduced from eleven to ten dimensions on a light-like circle $\Gamma y i e l d s$ the solution of ten-dimensional supergravity that is believed to be the macroscopic description of a D0-particle.

A massless test particle with the same $d$-velocity $\mathbf{u}$ as above feels a gravitational force that can be read off from the geodesic equation

$$
\frac{d u^{i}}{d \tau}=\Gamma_{\mu \nu}^{i} u^{\mu} u^{\nu}
$$

The Christophel symbols for the Aichelburg-Sexl are easily calculated

$$
\Gamma_{\mu \nu}^{i}=\frac{1}{2} g^{i \lambda}\left(g_{\mu \lambda, \nu}+g_{\nu \lambda, \mu}-g_{\mu \nu, \lambda}=-\frac{1}{2} \partial^{i} g_{\mu \nu}\right)
$$

Again $\Gamma$ there is only one non-zero combination of indices $\Gamma$ namely $\Gamma_{--}^{i}$ and the acceleration becomes

$$
\frac{d u^{i}}{d \tau} \propto u^{-} u^{-} \partial^{i} \frac{\delta\left(x^{-}\right)}{\left\|x_{\perp}\right\|^{d-4}} \propto v^{4} \partial^{i} \frac{\delta\left(x^{-}\right)}{\left\|x_{\perp}\right\|^{d-4}}+O\left(v_{\perp}^{6}\right)
$$

To leading order $\Gamma$ we again find a velocity dependent potential

$$
V \propto \frac{v_{\perp}^{4}}{\left\|x_{\perp}\right\|^{7}}
$$

if we evaluate the expression for eleven-dimensional supergravity. In contrast to the potential in the Maxwell theory scaling with $v^{2}$ Tin gravity like $\left(v^{2}\right)^{2}$. This is a manifestation of a phenomenon that is well known in string theory: Scattering amplitudes (we have calculated an effective potential but this is closely related to a scattering amplitude by the LSZ-formula) in gravity are often expressible as squares of gauge theory scattering amplitudes due to the fact that the former are described by closed strings with two independent sets of oscillator modes whereas the latter ones are obtained from open strings with just one independent set of modes[GSW].

If we are interested in higher orders in $v_{\perp}$ Twe should be careful to note that in the Aichelburg-Sexl background (4.4) is no longer light-like but we have to take into account 
corrections. Therefore $\Gamma$ in that case $\Gamma$ it is more convenient to follow the approach of [BBPT]. Here we normalize $\mathbf{u}$ by setting $u^{+}=1$. This corresponds to parameterizing the world-line by $\tau=x^{+}$as it is usual in light-cone frame calculations. Then $\Gamma$ the condition for $\mathbf{u}$ being null is

$$
2 u^{-}+v_{\perp}^{2}+h_{--} u^{-} u^{-}=0 .
$$

This can be solved for

$$
u^{-}=\frac{\sqrt{1-h_{--v_{\perp}^{2}}}-1}{h_{--}}
$$

Now $\Gamma$ we should note that the M(atrix)-Model assumes constant $p_{-}$Tnot $u^{-}$. Therefore we should consider the Legendre transform of $L$ with respect to $u^{-}$. As the Lagrangian of a massless particle is zero we find (we set $p_{-}=1$ as we consider the field of one D0-particle)

$$
\begin{aligned}
L^{\prime} & =L-p_{-} u^{-} \\
& =\frac{v^{2}}{2}+\frac{1}{8} \frac{\alpha v^{4}}{r^{d-4}}+\frac{1}{16} \frac{\alpha^{2} v^{6}}{r^{2(d-4)}}+O\left(v^{8}\right) .
\end{aligned}
$$

It was one of the original supporting arguments for the $\mathrm{M}$ (atrix)-Theory conjecture in [BFSS] that not only the form of the potentials (4.2) and (4.6) matches but also their numerical coefficient $-15 / 16$ (it turns out $\alpha=\frac{15}{2}$ is really the correct normalization for the Aichelburg-Sexl metric in order for the solution $\Gamma$ when reduced to ten dimensions $\Gamma$ carries one unit of D0 charge). This was taken as strong evidence for the conjecture that eleven-dimensional M-Theory is well described by the quantum mechanics of the supersymmetric matrix model. It is the main purpose of this thesis to investigate whether this correspondence persists in further Tmore detailed comparisons of scattering processes.

\subsection{Spin-Spin Interaction in M(atrix)-Theory}

As it should have become clear in the preceeding section $\Gamma$ the calculation of the leading order force on the test-particle in the Aichelburg-Sexl metric did not involve any properties of the test particle. The force we found is universal and independent of the kind of particle and of possible orientations and polarizations. To support the assumption that the particles described by the diagonal degrees of freedom of the matrices should be identified with the particles of the graviton multiplet of eleven-dimensional supergravity $\Gamma$ we need to test properties specific to gravitons like the spin being $s=2$ and resulting polarization states. Therefore in this section Tour aim is to calculate the part of the potential that is maximally dependent on the polarization of the scattering particles. In analogy to atomic physics $\Gamma$ we interpret it as the spin-spin interaction. The presentation here will closely follow the one given in $[\mathbf{B H P}]$.

As it is known since the early days of the supermembrane[dWHN] $\Gamma$ the $128+128$ dimensional graviton multiplet of maximal supergravity arises in the M(atrix)-Model by acting on some ground state wave function with the eight fermionic creation operators 
built out of the fermionic matrices. Thus $\Gamma$ the different states of graviton polarization are accessible in terms of expectations involving the fermionic fields.

We are going to look for these spin dependent interactions in the terms in the effective potential that are related by a supersymmetry transformation to the $v^{4} / r^{7}$ term we calculated before. But instead of applying the possibly one-loop modified supersymmetry transformations on this term $\Gamma$ we are going to calculate the effective action directly $\Gamma$ again with the help of the background field formalism.

Power-counting tells us that we can expect to find each factor of $v$ possibly being replaced by $\psi^{2} / r$. The maximally spin dependant term will therefore be of order $\psi^{8} / r^{11}$. Because of the supersymmetry cancellations we encountered for the purely bosonic term $\Gamma$ there should not be any static terms with less than eight fermions. This will indeed be the result we will derive in this section.

Before we start with the actual calculation let us state some $\gamma$-matrix identities. We use a representation of the $S O(9)$ Clifford algebra by real $\Gamma$ symmetric matrices[GSw]. From this it follows that products of two or three $\gamma$-matrices are anti-symmetric in the spinor indices whereas products of zeroГone $\Gamma$ four $\Gamma$ and five are symmetric. Thus $\Gamma$ we have for an anti-commuting spinor $\theta$

$$
\begin{array}{rlrl}
\theta \theta & =0 & \theta \gamma^{i} \theta=0 \\
\theta \gamma^{i} \gamma^{j} \theta & =\theta \gamma^{i j} \theta & \theta \gamma^{i} \gamma^{j} \gamma^{k} \theta=\theta \gamma^{i j k} \theta \\
\theta \gamma^{i} \gamma^{j} \gamma^{k} \gamma^{l} \theta & =\delta^{i j} \theta \gamma^{k l}-\delta^{i k} \theta \gamma^{j l}+\delta^{i l} \theta \gamma^{j k}+\delta^{j k} \theta \gamma^{i l}-\delta^{j l} \theta \gamma^{i k}+\delta^{k l} \theta \gamma^{i j} .
\end{array}
$$

In $S O(9)$ there is one Fierz identity that is a remnant of $S O(8)$ triality: Any anti-symmetric matrix in spinor indices can be decomposed as

$$
\theta_{\alpha} \theta_{\beta}=\frac{1}{32} \theta \gamma^{i j} \theta \gamma_{\alpha \beta}^{i j}+\frac{1}{96} \theta \gamma^{i j k} \theta \gamma_{\alpha \beta}^{i j k}
$$

This can be used to derive a couple of identities that we will need later on. We list them here without much further ado[BHP]:

$$
\begin{aligned}
& \theta \gamma^{i j} \theta \theta \gamma^{i j} \theta=0
\end{aligned}
$$

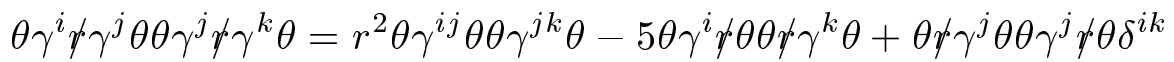

$$
\begin{aligned}
& -r^{i} \theta r \gamma^{j} \theta \theta \gamma^{j k} \theta-r^{k} \theta \gamma^{i j} \theta \theta \gamma^{j} r \theta
\end{aligned}
$$

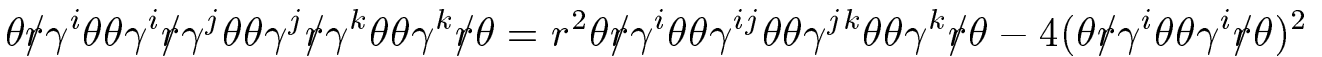

$$
\begin{aligned}
& \theta \gamma^{i j} \theta \theta \gamma^{j} \cdot \gamma^{k} \theta \theta \gamma^{k} \not \gamma^{l} \theta \theta \gamma^{l i} \theta=r^{2} \theta \gamma^{i j} \theta \theta \gamma^{j k} \theta \theta \gamma^{k l} \theta \theta \gamma^{k i} \theta \\
& -7 \theta \gamma \gamma^{i} \theta \theta \gamma^{i j} \theta \theta \gamma^{j k} \theta \theta \gamma^{k} \gamma \theta \\
& \theta \gamma^{i} \gamma^{j} \theta \theta \gamma^{j} \gamma^{k} \theta \theta \gamma^{k} \not \gamma^{l} \theta \theta \gamma^{l} \not \gamma^{i} \theta=r^{4} \theta \gamma^{i j} \theta \theta \gamma^{j k} \theta \theta \gamma^{k l} \theta \theta \gamma^{k i} \theta \\
& -12 r^{2} \theta \gamma \gamma^{i} \theta \theta \gamma^{i j} \theta \theta \gamma^{j k} \theta \theta \gamma^{k} r \theta \\
& +22\left(\theta r \gamma^{i} \theta \theta \gamma^{i} r \theta\right)^{2} \\
& \theta \gamma^{i j} \theta \theta \gamma^{j} \not \gamma^{k} \theta \theta \gamma^{k l} \theta \theta \gamma^{l} \not \gamma^{i} \theta=r^{2} \theta \gamma^{i j} \theta \theta \gamma^{j k} \theta \theta \gamma^{k l} \theta \theta \gamma^{k i} \theta \\
& -8 \theta \gamma \gamma^{i} \theta \theta \gamma^{i j} \theta \theta \gamma^{j k} \theta \theta \gamma^{k} \gamma \theta
\end{aligned}
$$


If we adhered strictly to the background field formalism we should consider fermionic background fields being turned on in order to find fermionic terms in the effective action. But those would result in boson-fermion mixing terms arising from the Yukawa interaction. One would have to diagonalize a mass matrix with fermionic entries. In order to circumvent such difficulties एwe decide to treat only the bosonic part of the background with the help of the background field formalism and to calculate diagrams with external fermion lines.

Because we are only interested in the static term $\Gamma$ we can set $v=0$. This removes all explicit time dependence of the background Lagrangian and we can therefore easily calculate diagrams in momentum representation rather than in position space representation to where one has to resort so as to treat non-constant backgrounds.

As we will see there will only be off-diagonal fields running in the loop because the background fields are all diagonal and because there can be only one diagonal field at the cubic vertices. Therefore $\mathrm{Tit}$ is convenient to parameterize the matrices as follows:

$$
\begin{gathered}
\mathbf{X}^{i}=\frac{i}{2}\left(\begin{array}{cc}
X^{i} & \sqrt{2} \bar{Y}^{i} \\
\sqrt{2} Y^{i} & -X^{i}
\end{array}\right) \quad \mathbf{A}=\frac{i}{2}\left(\begin{array}{cc}
A & \sqrt{2} \bar{B} \\
\sqrt{2} B & -A
\end{array}\right) \\
\mathbf{\Psi}=\frac{i}{2}\left(\begin{array}{cc}
\theta & \sqrt{2} \bar{\psi} \\
\sqrt{2} \psi & -\theta
\end{array}\right)
\end{gathered}
$$

In terms of these components $\Gamma$ the relevant parts of the Lagrangian read:

$$
\begin{aligned}
& L_{Y}=-\bar{Y}^{i}\left(\partial_{t}^{2}+r^{2}\right) Y^{i}+(\text { interactions }) \\
& L_{B}=\bar{B}\left(\partial_{t}^{2}+r^{2}\right) B+(\text { interactions }) \\
& L_{\psi}=\frac{1}{2} \bar{\psi}\left(i \partial_{t}-\not\right) \psi+\bar{\psi} Y \theta+\theta \bar{Y} \psi-B \bar{\psi} \theta-\bar{B} \theta \psi+\text { (interactions) }
\end{aligned}
$$

The ghosts can be ignored as they do not couple to the fermions directly. We can directly read off the Feynman rules. We denote the gauge field by a dashed line $\Gamma$ the scalars by a wavy line and the fermions by a solid line. The propagators are

$$
\begin{aligned}
& \left\langle\bar{Y}^{i} Y^{j}\right\rangle=j \leadsto i=\frac{i \delta^{i j}}{\omega^{2}-r^{2}+i \epsilon} \\
& \langle\bar{B} B\rangle=-\cdots---=\frac{-i}{\omega^{2}-r^{2}+i \epsilon} \\
& \left\langle\bar{\psi}_{\alpha} \psi_{\beta}\right\rangle=\beta \longrightarrow \alpha=\frac{i(\omega+\not)_{\alpha \beta}}{\omega^{2}-r^{2}+i \epsilon}
\end{aligned}
$$

The vertices for the Yukawa interactions are
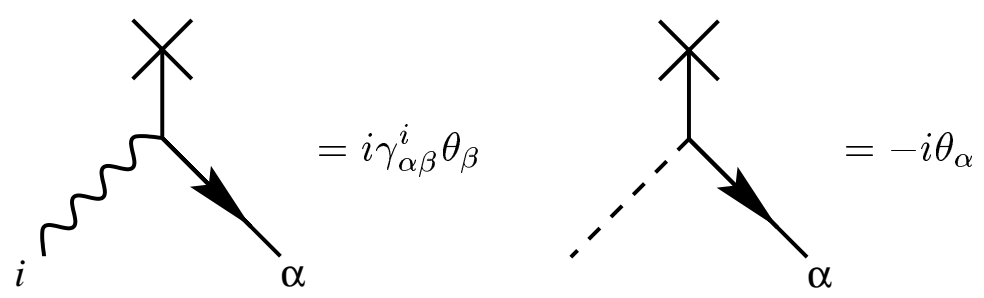
We first consider diagrams with two external fermions. There can either be a gauge field or a scalar running in the loop. The diagram with the gauge field is easily found to vanish because of the anti-symmetry of the $\gamma$-matrices:

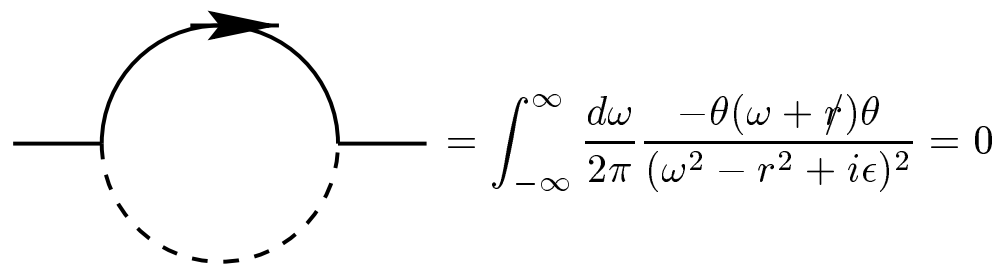

The diagram with a scalar particle is zero

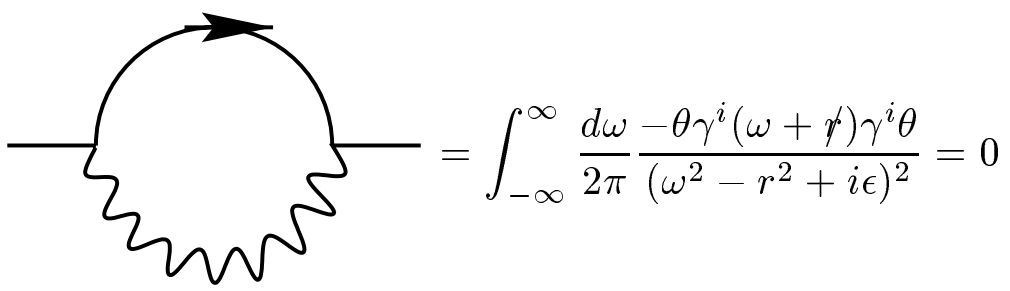

Both of the above diagrams are traversed by only one fermion. We will call any series of fermion traversals connected by $Y$ fields a chain. A chain is ended by the gauge field or it connects back to itself (in case the loop does not contain any gauge fields). As an example $\Gamma$ there are four $\theta^{6}$ diagrams shown in Fig. 7. The first contains three chains with only a single fermion traversion each (one link chains). The last two contain three link chains.
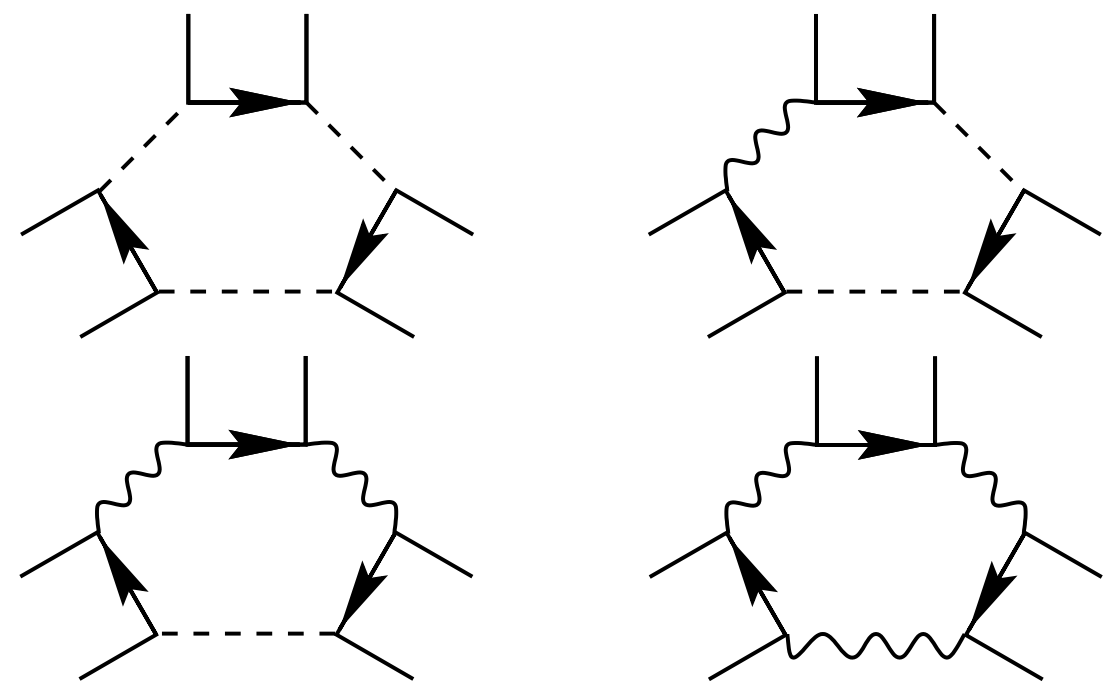

Fig. 7: The $\theta^{6}$ diagrams

Here $\Gamma$ we prove that any diagram containing a chain with an odd number of links is zero. First $\Gamma$ consider a closed chain with $n$ links. Before doing the $\omega$ integral $\Gamma$ it will 
contain a factor

$$
\theta \gamma^{i_{1}}(\omega+\not r) \gamma^{i_{2}} \theta \theta \gamma^{i_{2}}(\omega+\not r) \gamma^{i_{3}} \theta \cdots \theta \gamma^{i_{n}}(\omega+\not r) \gamma^{i_{1}} \theta
$$

Each fermion bilinear is anti-symmetric in its $S O(9)$ indices. Since there is an odd number of factors $\Gamma$ swapping the indices on all of them produces an overall minus sign.

$$
\left.\left.-\theta \gamma^{i_{2}}(\omega+\not)\right) \gamma^{i_{1}} \theta \theta \gamma^{i_{3}}(\omega+\not)\right) \gamma^{i_{2}} \theta \cdots \theta \gamma^{i_{1}}(\omega+\not \gamma) \gamma^{i_{n}} \theta
$$

The factors can be reordered $\Gamma$

$$
-\theta \gamma^{i_{1}}(\omega+\not \gamma) \gamma^{i_{n}} \theta \theta \gamma^{i_{n}}(\omega+\not \gamma) \gamma^{i_{n-1}} \theta \cdots \theta \gamma^{i_{2}}(\omega+\not \gamma) \gamma^{i_{1}} \theta
$$

to reproduce after a relabeling of the contracted indices the negative of the original expression. This implies that this term is zero. Chains ended by gauge fields contribute a factor

$$
\theta \not \gamma^{i_{1}} \theta \theta \gamma^{i_{1}}(\omega+\not \gamma) \gamma^{i_{2}} \theta \cdots \theta \gamma^{i_{n-2}}(\omega+\not \gamma) \gamma^{i_{n-1}} \theta \theta \gamma^{n-1} \not \gamma
$$

which vanish for a similar reason. Therefore any diagram containing a chain with an odd number of links is zero. All diagrams that have an odd number of pairs of external fermion lines must contain a chain with an odd number of links. For a diagram to give a non-vanishing contribution $\Gamma$ the number of external $\theta$ lines must be a multiple of four. Via supersymmetry this corresponds to our earlier finding that bosonic diagrams with an odd number of factors of $v$ have to vanish by time-reversal symmetry.

After what we have said so far $\Gamma$ the $\theta^{4}$ term could be non-vanishing. But it also can seen to be zero as follows: First Tone diagram is zero because it contains odd chains:

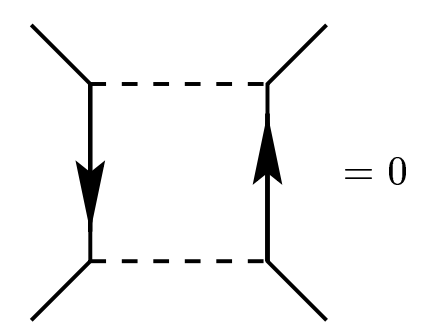

The cancellation of the remaining two diagrams needs two of the identities we listed above $\Gamma$ namely (4.7) and (4.8):

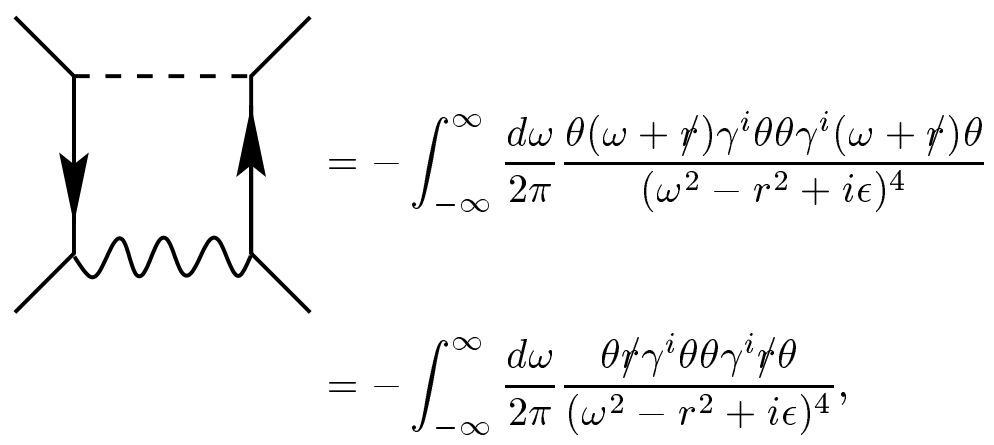


and including a factor of $\frac{1}{2}$ for the twofold symmetry of the diagram:

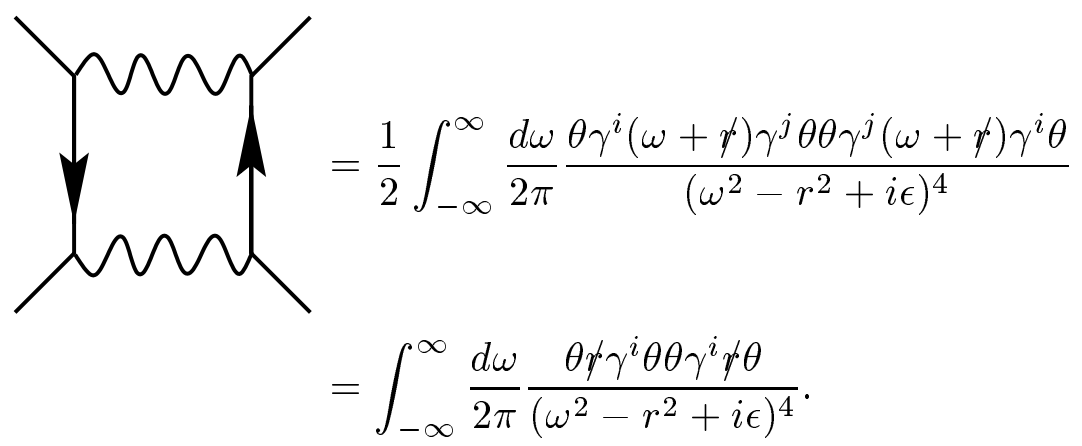

The vanishing of this term is again related $\Gamma$ via supersymmetry 5 to the vanishing of a purely bosonic term Tnamely $v^{2} / r^{5}$.

The $\theta^{8}$ term should give the first non-zero contribution since it corresponds to the $v^{4} / r^{7}$ term we calculated above. Again $\Gamma$ there are three diagrams that vanish due to odd chains:
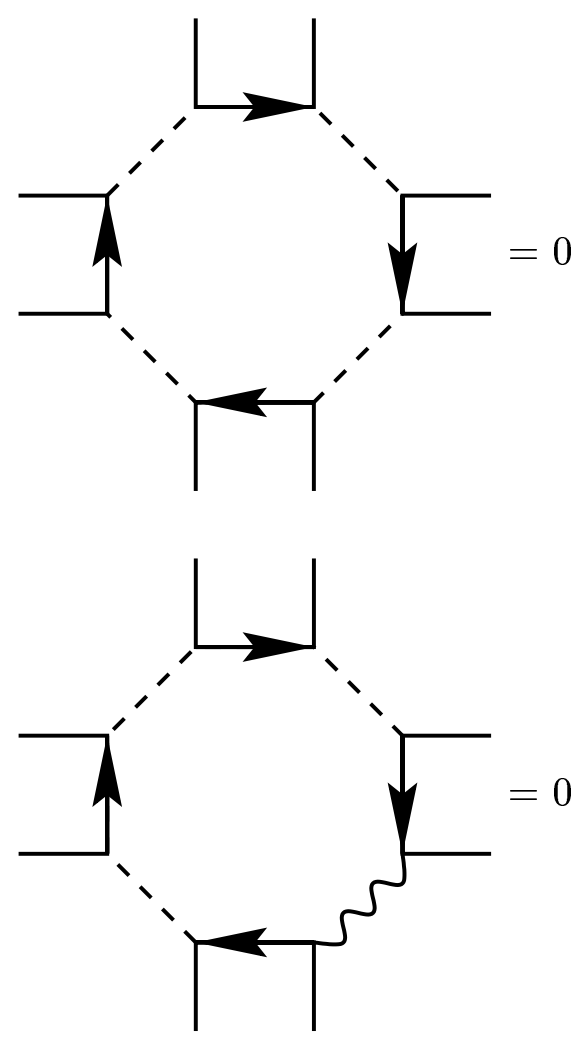


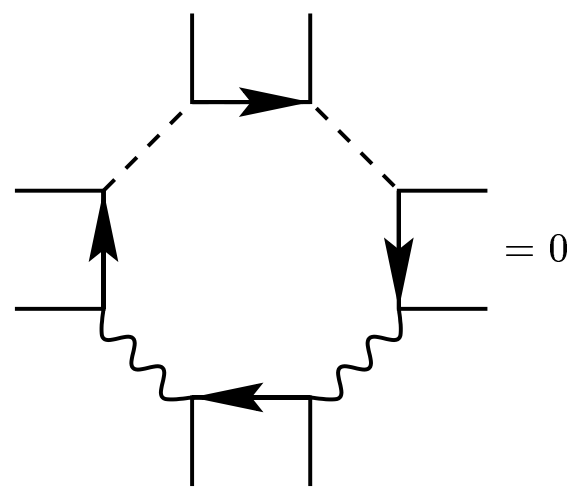

To calculate the remaining diagrams $\Gamma$ we will need the following numerical integrals:

$$
\begin{aligned}
& \int_{-\infty}^{\infty} \frac{d \omega}{2 \pi} \frac{1}{\left(\omega^{2}-r^{2}+i \epsilon\right)^{8}} \stackrel{\epsilon \rightarrow 0}{=} \frac{429 i}{4096 r^{15}} \\
& \int_{-\infty}^{\infty} \frac{d \omega}{2 \pi} \frac{\omega^{2}}{\left(\omega^{2}-r^{2}+i \epsilon\right)^{8}} \stackrel{\epsilon \rightarrow 0}{=} \frac{33 i}{4096 r^{13}} \\
& \int_{-\infty}^{\infty} \frac{d \omega}{2 \pi} \frac{\omega^{4}}{\left(\omega^{2}-r^{2}+i \epsilon\right)^{8}} \stackrel{\epsilon \rightarrow 0}{=} \frac{9 i}{4096 r^{11}}
\end{aligned}
$$

The first diagram contains two chains of length two. Again of 2 for over-counting the rotational symmetry of the diagram:

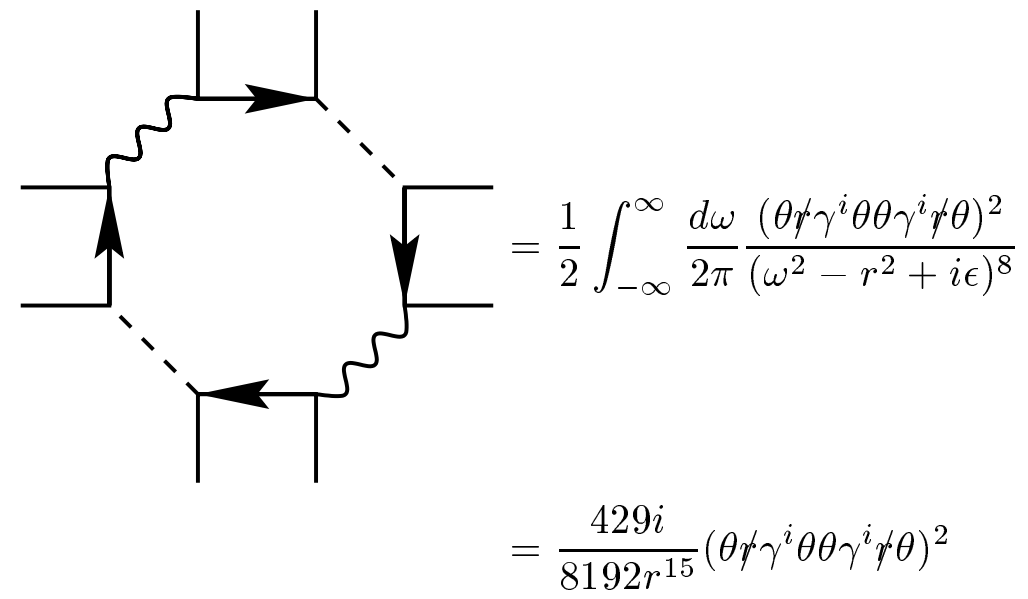

To simplify the two final diagrams $\Gamma$ we employ identities $(4.9) \Gamma(4.10) \Gamma(4.11) \Gamma$ and $(4.12)$. 
With these we obtain

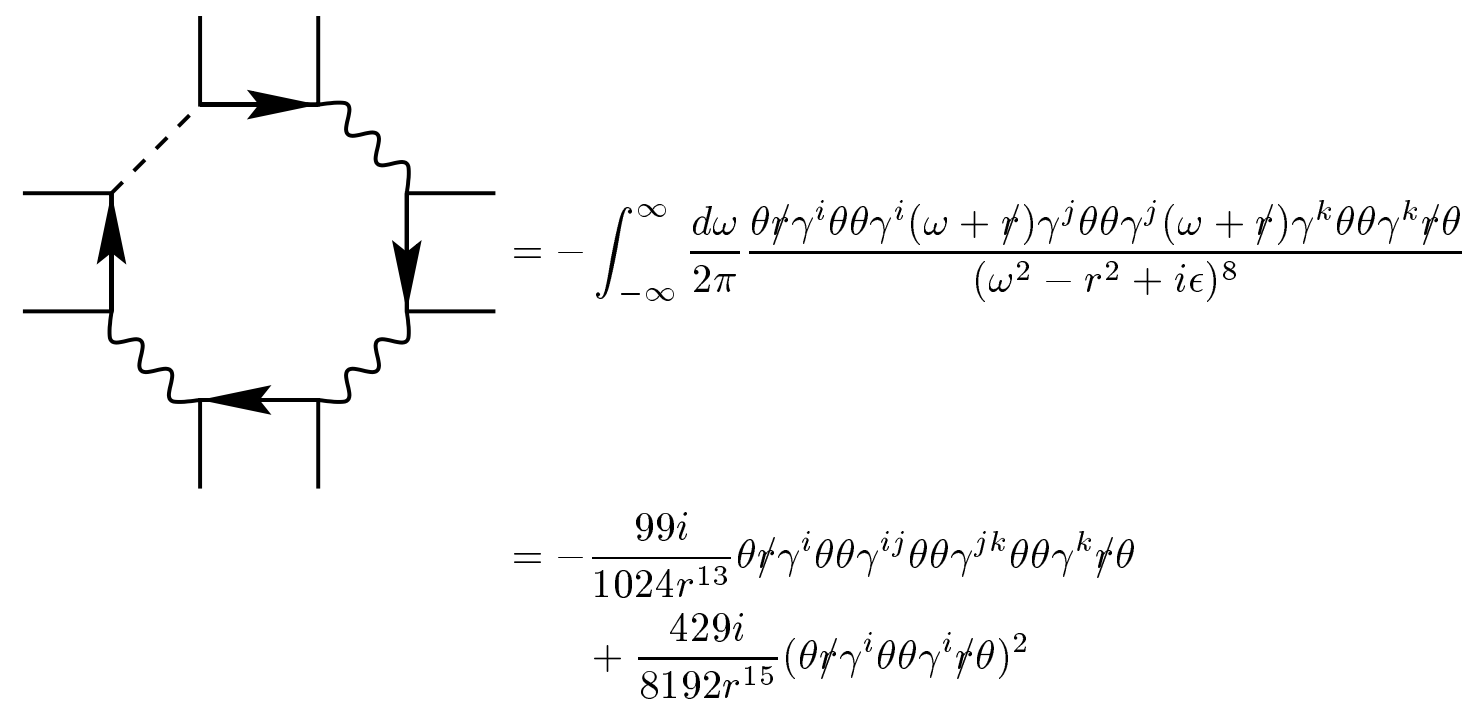

and finally including a compensation for the fourfold symmetry

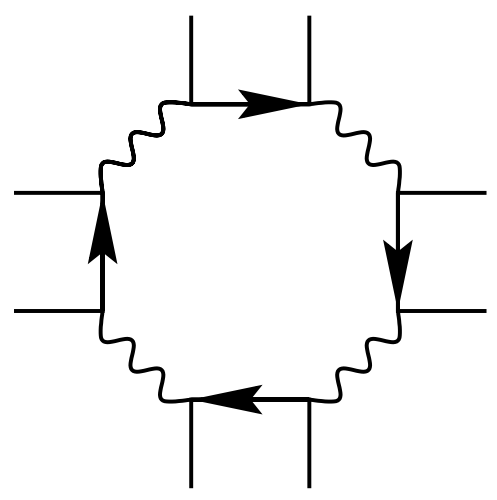

$$
\begin{aligned}
& =\frac{1}{4} \int_{-\infty}^{\infty} \frac{d \omega}{2 \pi} \frac{\theta \gamma^{i}(\omega+\not \gamma) \gamma^{j} \theta \theta \gamma^{j}\left(\omega+\not \gamma^{k}\right) \gamma^{k} \theta \theta \gamma^{k}(\omega+\not \gamma) \gamma^{l} \theta \theta \gamma^{l}(\omega+\not \gamma) \gamma^{i} \theta}{\left(\omega^{2}-r^{2}+i \epsilon\right)^{8}} \\
& =\frac{15 i}{1024 r^{11}} \theta \gamma^{i j} \theta \theta \gamma^{j k} \theta \theta \gamma^{k l} \theta \theta \gamma^{l i} \theta \\
& -\frac{231 i}{1024 r^{13}} \theta r^{i} \theta \theta \gamma^{i j} \theta \theta \gamma^{j k} \theta \theta \gamma^{k} r \theta \\
& +\frac{4719 i}{8192 r^{15}}\left(\theta r \gamma^{i} \theta \theta \gamma^{i} \gamma^{2} \theta\right)^{2}
\end{aligned}
$$

Summing up all the above diagrams $\Gamma$ we find the effective potential at order $\theta^{8} / r^{11}$ :

$$
\begin{aligned}
V(r, \theta)= & -\frac{15}{(2 r)^{11}}\left(2 \theta \gamma^{i j} \theta \theta \gamma^{j k} \theta \theta \gamma^{k l} \theta \theta \gamma^{l i} \theta-\frac{44}{r^{2}} \theta r \gamma^{i} \theta \theta \gamma^{i j} \theta \theta \gamma^{j k} \theta \theta \gamma^{k} \gamma \theta\right. \\
& \left.+\frac{143}{r^{4}}\left(\theta \gamma \gamma^{i} \theta \theta \gamma^{i} \gamma^{2} \theta\right)^{2}\right) \\
= & -\frac{5}{43008}\left(\theta \not \partial \gamma^{i} \theta \theta \gamma^{i} \not \partial \theta\right)^{2} \frac{1}{r^{7}}
\end{aligned}
$$


There may be terms of higher order in $\theta$. Since $\theta$ only has sixteen components $\Gamma$ the only two terms that could remain would be proportional to $\theta^{12} / r^{17}$ or $\theta^{16} / r^{23}$. These are related to the higher order terms $\frac{v^{6}}{r^{11}}$ and $\frac{v^{8}}{r^{15}}$ which we also suppressed above. It is remarkable that the contributions from the diagrams conspire to give exactly the coefficient that one gets from acting on $1 / r^{7}$ with four gradients.

In order to make contact with supergravity $\Gamma$ we take the Fourier transform in the nine transversal directions:

$$
V(q, \theta)=\frac{\pi^{4}}{252} \frac{\left(q^{i} J^{i j} J^{j k} q^{k}\right)^{2}}{q^{2}}
$$

where we have inserted in the relative angular momentum $J^{i j}=\frac{i}{2} \theta \gamma^{i j} \theta$ of the D0particles $[\mathbf{K}]$.

\subsection{Spin-Spin Interaction in Gravity and String Theory}

To make contact with the gravity interpretation of the process $\Gamma$ we follow $[\mathbf{H}]$. There $\Gamma$ it is found that the calculation of the polarization dependent terms of the effective action in gravity is most easily performed using string theory to keep track of the various kinematic indices. We will use coordinates that that are explicitly covariant under the $S O(9)$ of the transversal coordinates.

The process we consider is the scattering of two Ramond-Ramond particles in ninedimensional type II theory. The calculation will be performed in the Green-Schwarz formalism. Amplitudes of gravity arise from scattering closed strings. Since we try to match four factors of $J$ describing graviton polarizations we are interested in a closed string fourpoint function. As we already mentioned above $\Gamma$ those can be constructed by multiplying open string amplitudes for the left and right moving modes $\Gamma$ concretely

$$
A_{4}^{c l}=\kappa^{2} \zeta_{1}^{A A^{\prime}} \zeta_{2}^{B B^{\prime}} \zeta_{3}^{C C^{\prime}} \zeta_{4}^{D D^{\prime}} K_{A B C D}^{L}(k / 2) K_{A^{\prime} B^{\prime} C^{\prime} D^{\prime}}^{R}(k / 2) C(s, t, u),
$$

where the $\zeta_{a}$ are polarization tensors of the external particles $\Gamma C(s, t, u)$ is a function of the Mandelstam variables that $\Gamma$ in our case of small relative velocities $v$ and vanishing momentum transfer $q$ Treduces to $\pi / 8 q^{2}$ and the $K$ 's are kinematical factors of open string four point functions.

As we are going to scatter Ramond-Ramond fields that are made up of tensor products of left- and right-moving spinors $\Gamma$ the relevant open string process is the scattering

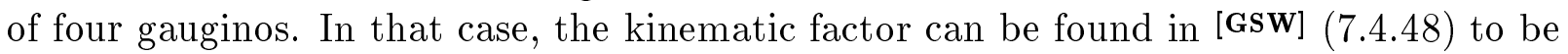

$$
K\left(u_{1}, u_{2}, u_{3}, u_{4}\right)=-\frac{1}{2} s \bar{u}_{2} \Gamma^{\mu} u_{3} \bar{u}_{1} \Gamma_{\mu} u_{4}+\frac{1}{2} t \bar{u}_{1} \Gamma^{\mu} u_{2} \bar{u}_{4} \Gamma_{\mu} u_{3} .
$$


Here $\Gamma$ the $u_{a}$ are the wave functions of the external fermions. In our case of eikonal scattering we can ignore the term proportional to $t=q^{2}$. For the $S O(9,1) \Gamma$-matrices $\Gamma$ following $[\mathbf{H}] \Gamma$ we use

$$
\Gamma^{0}=i\left(\begin{array}{cc}
0 & 1 \\
-1 & 0
\end{array}\right) \quad \Gamma^{m}=\left(\begin{array}{cc}
0 & \bar{\gamma}^{m} \\
\bar{\gamma}^{m} & 0
\end{array}\right)
$$

with $S O(9)$ matrices

$$
\bar{\gamma}^{i}=i\left(\begin{array}{cc}
0 & \gamma^{i} \\
\gamma^{i} & 0
\end{array}\right) \quad \bar{\gamma}^{9}=i\left(\begin{array}{cc}
1 & 0 \\
0 & -1
\end{array}\right)
$$

where $S O(8)$ matrices from [GSW] were used. The external wave functions have to be on-shell which is equivalent to saying they have to obey the Dirac equation

$$
\Gamma^{\mu} p_{\mu} u(p)=0 \text {. }
$$

This can be achieved by writing the sixteen component spinor $u^{L / R}$ as the transpose of two eight component spinors $\left(\lambda^{L / R}, 0\right)$ and in the rest frame $\Gamma$ where $p$ has only components in the 0 and 9 direction $\Gamma$ by further requiring

$$
-i \bar{\gamma}^{9} \lambda^{L / R}=\mp \lambda^{R / L} \text {. }
$$

We can conclude that in the rest frame

$$
\lambda^{L}=\sqrt{M / 2}\left(\begin{array}{c}
\xi_{a}^{L} \\
0
\end{array}\right) \quad \lambda^{R}=\sqrt{M / 2}\left(\begin{array}{c}
0 \\
\xi_{\dot{a}}^{R}
\end{array}\right) .
$$

Now we boost the incoming spinors to velocity $v$ and the outgoing spinors to $w$. For incoming particle $I$ we find

$$
\lambda_{I}^{L}=\sqrt{M / 2}\left(\begin{array}{c}
\left(1+v^{2} / 4\right) \xi_{I}^{L} \\
-(\vec{V} \cdot \vec{\gamma} / 2) \xi_{I}^{L}
\end{array}\right),
$$

with similar expressions for outgoing particles and right moving spinors. Defining the center of mass frame momentum $\vec{k}=M(\vec{v}+\vec{w})$ and momentum transfer $\vec{q}=M(\vec{v}-\vec{w}) \Gamma$ one finally arrives at

$$
K^{L}\left(u_{1}^{L}, u_{2}^{L}, u_{3}^{L}, u_{4}^{L}\right)=\frac{s-4 M^{2}}{8}\left(\frac{\vec{k}^{2}}{8}+\frac{5}{16} k_{i} q_{j}\left(J_{1 L}^{i j}+J_{2 L}^{i j}\right)-\frac{1}{16} q_{j} q_{k} J_{1 L}^{i j} J_{2 L}^{i k}\right) .
$$

Thus Tone finds the closed string amplitude to be

$$
A_{4}=-\frac{\pi \kappa^{2}}{8 \vec{q}^{2}}\left(\vec{k}^{2}+\frac{5}{2} k_{i} q_{j}\left(J_{1}^{i j}+J_{2}^{i j}\right)-\frac{1}{2} q_{j} q_{k} J_{1}^{i j} J_{2}^{i k}\right)^{2}
$$

where we have introduced $J_{I}^{i j}=\xi_{I}^{\dagger} \gamma^{i j} \xi_{I} / 4$. Obviously $\Gamma$ the first term squared is just the bosonic $v^{4} / r^{7}$ potential while the last term squared matches the spin-spin interaction we have calculated above by means of the M(atrix)-Model. The other terms are various spinorbit interactions most of which have also been calculated from the matrix perspective using different methods. The leading spin-orbit term was first found in $[\mathbf{K}]$ by calculating a loop with two external fermions using the background propagator (4.1) $\Gamma$ whereas [McA] calculates the $v^{2} \psi^{4} / r^{9}$ term using perturbative heat-kernel methods. Up to date $\mathrm{there}$ is no direct perturbative calculation of the six fermion term in the M(atrix)-ModelГalthough its form and coefficient have been found using supersymmetry. 


\subsection{Understanding the Correspondence}

In the previous section $\Gamma$ we found a strong connection between the M(atrix)-Model and eleven-dimensional supergravity. More specifically we found we could calculate the leading order tree-level (corresponding to classical supergravity) scattering amplitudes also using the M(atrix)-Model at one loop-order.

If we look at this process from a stringy point of view $\Gamma$ the process is described by the following diagram:

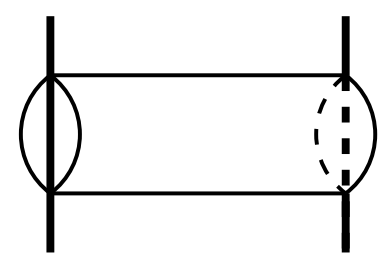

As a tree level gravity amplitude $\mathrm{Cit}$ can be viewed as the exchange diagram of a closed string. As a gauge theory diagram $\Gamma i t$ can be viewed as a one loop diagram of open strings. From the latter point of view $\Gamma$ the diagram is more conveniently drawn as the annulus diagram

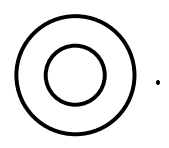

In the gauge theory language $\Gamma$ this can be viewed as a Feynman diagram using 't Hooft's ribbon notation where gauge fields are represented by ribbons with the closed lines indicating the contraction of matrix indices.

This world-sheet duality already makes the correspondence less mysterious. As it was already noted in [DKPS] in this specific process $\Gamma$ world-sheet duality is even stronger then in the generic case $\Gamma$ since in the explicit calculation of the diagram one finds that only the world-sheet zero modes representing the massless fields of supergravity and gauge theory contribute to the amplitudes; all the higher excitations decouple. In [MSS] it was argued that this renders the amplitude scale independent. In fact $\Gamma$ just looking at the topology of the string diagram is too naive because one should expect the open string description to be appropriate for D0-particles that are very close such that the stretching open strings are shorter than the string length

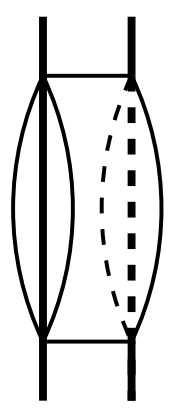

Fig. 8: The open string regime 
On the other hand $\Gamma$ the closed string description is more appropriate when the D0particles are separated by distances larger than the string length.

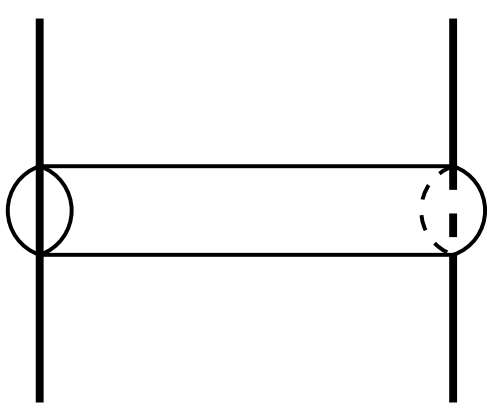

Fig. 9: The closed string regime

Even if world-sheet duality is not a proof of equality of open and closed string amplitudes in the low energy effective limit that is considered here (gauge theory and gravity respectively) where one only considers the zero modes $\Gamma$ it can be a very useful tool to determine which amplitudes have a chance to be related to each other. For example the diagram above we see that two Tthe number of $\mathrm{D} 0$-particles and therefore the number of boundaries in the closed string picture is the number of index loops in the open string description. In particularГwe immediately recognize that one-loop diagrams in $\mathrm{M}$ (atrix)Theory are relevant for two particle scattering. Indeed $\Gamma$ one finds that if one calculates one-loop diagrams for $\mathrm{M}$ (atrix)-Theory with $N>2$ one finds again the same terms we calculated above describing the mutual interactions of all pairs of particles whereas genuine three particle interactions are visible only at the two loop level. The three particle interactions will be the topic of the next chapter.

But before we move on to the investigation of higher order interactions the most convincing reason for the agreement we found in this chapter: supersymmetry. After the at first surprising connection between the matrix model and supergravity was found it was stressed that both theories have an extremely large amount of supersymmetry that severely constrains possible interactions. This was pointed out especially after Becker and Becker found in $[\mathbf{B B}]$ that the two particle interaction is not modified at the two loop level although power-counting allows $v^{4}$ terms that are sub-leading in $1 / r$.

IndeedГPabanГSethiTand Stern showed in [PSS1] that the complete fermion structure at the one-loop level can be derived from the $v^{4} / r^{7}$ term using supersymmetry alone. Later $\Gamma$ in $[\mathbf{H K S}]$ all terms in the effective action together with possible one-loop corrections to the supercharges that are known only on-shell were derived. Nicolai and Plefka showed recently[NP1] that the one-loop effective action of the M(atrix)-Model can be written in the form

$$
\begin{aligned}
L_{\mathrm{eff}} & =\exp \left(\frac{i}{2 v^{2}} \theta \psi \not \partial \theta\right) g(r, v) \\
& =g\left(r^{i}-\frac{i}{2 v^{2}} \theta \psi \not \partial \theta, v\right)
\end{aligned}
$$


up to total derivatives and an arbitrary function $g$ Tif one takes $v$ and $\theta$ to be constant in time. Any Lagrangian of this form is invariant under the original supersymmetry variations. If one further requires the action to be regular at $v=0 \Gamma$ the constant term in a Taylor expansion of $g$ around $v^{2}=0$ is absent $\Gamma$ whereas the linear term is the action of a super-particle and the quadratic term is the one-loop effective action of which we calculated the bosonic and maximal spin dependent term above. This should be viewed as a non-renormalization theorem for the two particle interaction that is crucial for the connection with supergravity not to be destroyed by higher loop corrections that modify the $1 / r^{7}$ dependence of the $v^{4}$ term. Anticipating a result form the next section $\Gamma$ we note already at this place that in [PSS2] a further non-renormalization theorem for two particle interactions at the order $v^{6}$ in velocity $\Gamma$ relevant at the two loop order $\Gamma$ was proven.

However it is important to note that these non-renormalization theorems are valid completely within the M(atrix)-Model and do not use directly the supersymmetry of the eleven-dimensional supergravity. Moreover $\Gamma$ the direct connection between the supersymmetries is not known and also as we saw Tthe loop expansion in both theories are different: Above we could compare a gauge theory loop diagram to a tree level diagram in gravity. 
$54 \ldots \ldots \ldots \ldots \ldots \ldots \ldots \ldots \ldots \ldots \ldots \ldots \ldots \ldots \ldots \ldots \ldots+$ The M(atrix)-Model at One-Loop Order 


\section{Two Loops and Three Particles}

In this chapter $\Gamma$ we go one order further. This will enable us to study processes with three particle interactions. First $\Gamma$ we will again compare M(atrix)-Model amplitudes to classical supergravity. But we will argue that at the two loop levelГquantum corrections to supergravity should be visible. We will investigate whether these corrections are reproduced by the M(atrix)-Model and find a negative answer!

\subsection{Power-Counting — The Rules of the Game}

Before we go into the details of higher loop matrix calculations $\Gamma$ we will have a closer look at the power-counting in this model in order to derive some simple rules that allow us to anticipate what kind of terms can be expected from detailed M(atrix)-Model calculations.

To start with let us once more look at the $v^{4} / r^{7}$ term to introduce the kinds of tricks we are going to play here. Let us pretend we calculate the diagram without the help of the background field formalism but with external legs and in momentum representation. We expect one-loop diagrams with four external $v$ lines to contribute. As there are no background fields $\Gamma$ the only bosonic vertices are four-point interactions coming from the $\left[X^{i}, X^{j}\right]^{2}$ term in the Lagrangian $\Gamma$ the natural diagram to consider is the one of "fish" type.

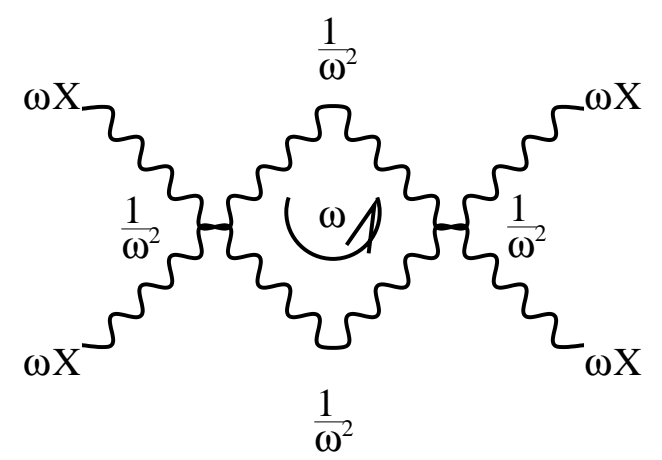

Fig. 10: The fish diagram contributing to $v^{4} / r^{7}$

There is one momentum $\omega$ running in the loop. The two bosonic propagators in the loop each contribute a factor of $\frac{1}{\omega^{2}}$. Ordinary external bosonic lines would contribute factors of $X$ rather than $v$. As we are interested in terms in the effective action involving $v$ 's Tone has to get a time derivative acting on the external line. This can be obtained in the integral expression for the diagram by partial integration. The effect is that the vertex has to be integrated in $t$ which is expressed as a multiplication by $\frac{1}{\omega}$ in the momentum representation. Therefore $\Gamma$ there will be four extra factors of $\frac{1}{\omega}$ for the four external lines. In conclusion $\Gamma$ the diagram will be proportional to

$$
v^{4} \int d \omega \frac{1}{\omega^{4}} \frac{1}{\omega^{4}} .
$$


This integral is infrared divergent. But we have not taken into account that there is a natural scale in the problem $\Gamma$ namely the impact parameter $b$ that can serve as a cut-off. It is therefore natural to evaluate the integral as

$$
v^{4} \int_{b}^{\infty} d \omega \frac{1}{\omega^{8}}=\frac{v^{4}}{b^{7}}=\frac{v^{4}}{r^{7}}
$$

where we substituted $r$ for $b$ by assuming the Lagrangian to be local in time. This interpretation of $b$ or $r$ as an IR cut-off can be justified from our more carful treatment in the previous chapter. There $\Gamma$ the particles running in the loop had masses at the order of $r$. This example shows that with this crude reasoning we can predict possible terms in the effective action. We will proceed by transforming this diagram by inserting further vertices and propagators to yield any possible M(atrix)-Theory diagram and read off the scaling with $r$ and $v$ as above. First $\Gamma$ we can insert further vertices with two external $v$ lines:

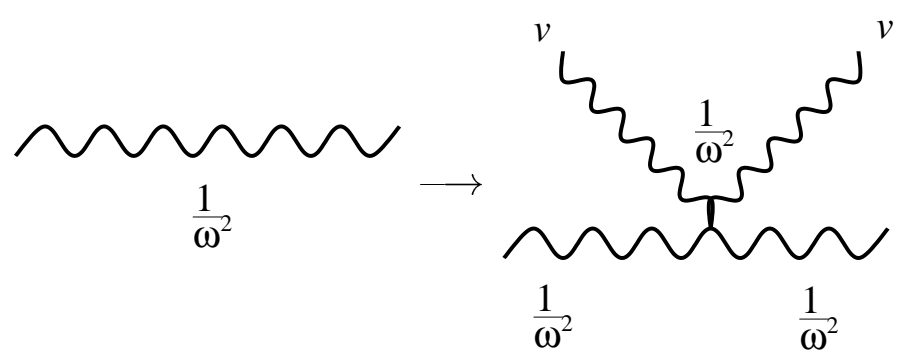

We have split one propagator into two thereby getting another factor of $\frac{1}{\omega^{2}}$ and added two new $v$ lines each of which yield another factor of $\frac{1}{\omega^{2}}$ because of the partial integration at the vertex. In total we have picked up a factor of

$$
\frac{v^{2}}{r^{4}}
$$

leaving the number of loops untouched. Note in passing $\Gamma$ that there can never be an odd number of $v$ 's in purely bosonic terms as there are only four-point vertices (with an even number of "ports") and internal lines connect two ports. Therefore $\Gamma$ there also have to be an even number of external lines connecting to the remaining ports. This agrees with the expectation of time reversal symmetry of the model.

The second kind of surgery we can do to a diagram is to insert a loop in a propagator:

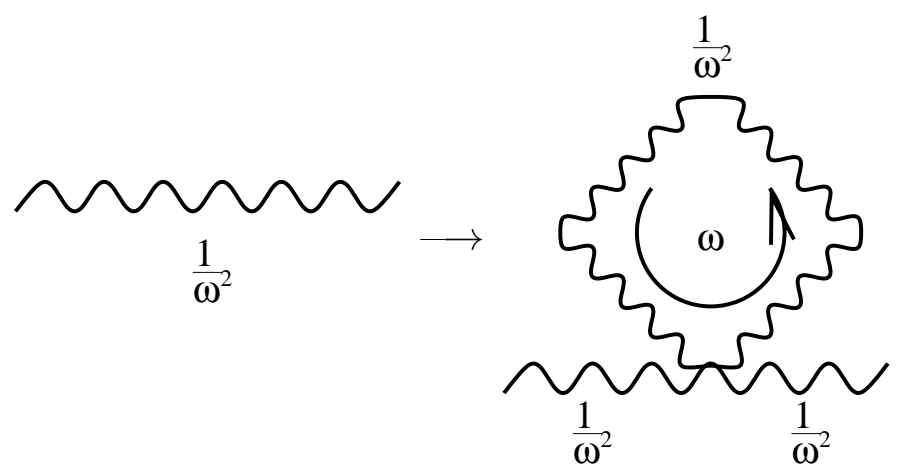


This also splits the original line into two another propagator supplying $\frac{1}{\omega^{2}}$ and also a new momentum integration $\int d \omega$ which can be counted as multiplying by $\omega$. Altogether $\Gamma$ the additional loop contributes

$$
\frac{1}{r^{3}}
$$

This reasoning is too crude to differentiate between the different loop momenta. For amplitudes with more than two particles involved $\Gamma$ there are more than just one distance scale $b$. One should expect a sum of different terms but the power-counting rules we derive here are not capable of resolving those.

The next modification of a diagram we are going to look at is the replacement of a bosonic loop by a fermionic loop. As the Yukawa interactions are only three-point vertices the bosonic four-point vertices have to be "resolved":

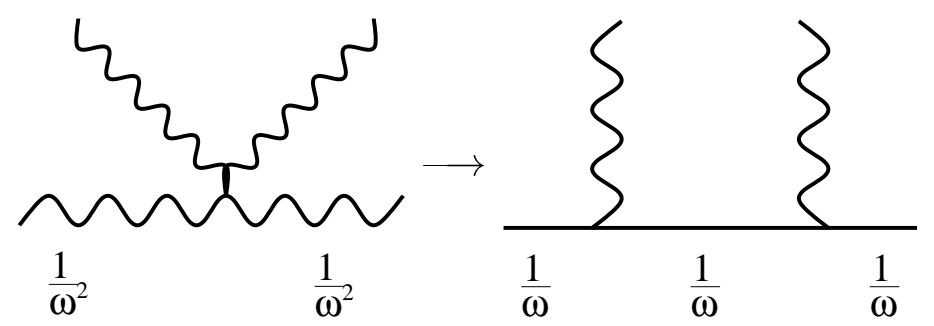

Thereby the number of propagators that form the loop doubles. But as a fermionic propagator contributes only $\frac{1}{\omega}$ the overall scaling of the diagram is not affected.

Finally we want to insert external fermions. Just like factors of $v$ in purely bosonic diagrams $\Gamma$ they always come in pairs. Thus we look at the insertion of a pair of fermions into a bosonic propagator as there are no vertices with more than two fermion lines:

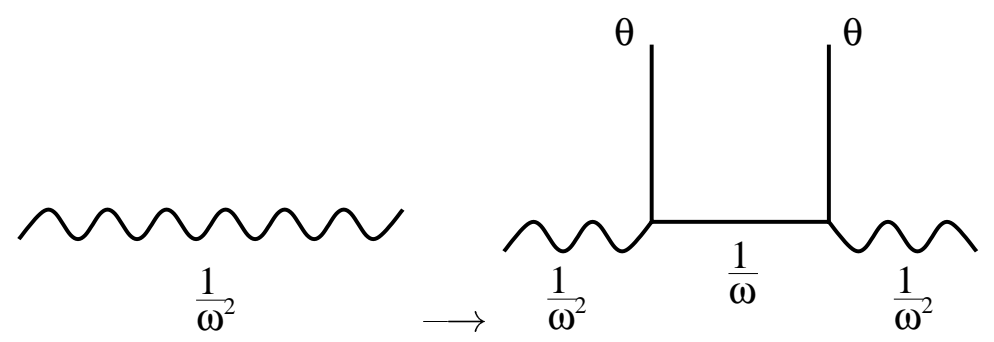

We have one new bosonic and one new fermionic propagator. In total $\Gamma$ we get a factor

$$
\frac{\theta^{2}}{r^{3}} \text {. }
$$

These operations allow us to obtain every diagram that is consistent with the Feynman rules of the M(atrix)-Model up to "topological" transformations that do not change the scaling. We can conclude that the effective action has to be of the form

$$
\Gamma=\sum_{L=\# \text { loops }} \sum_{I=\# v^{2} \text { insertions }} \sum_{F=\# \theta^{2} \text { insertions }} \frac{v^{2 I} \theta^{2 F}}{r^{4 I+3 L+3 F-4}}
$$


Consequently $\Gamma$ if we ignore fermionic external lines for a moment $\Gamma$ the effective action organizes itself as a table:

$$
\begin{aligned}
\Gamma= & v^{2} \\
& +c_{11} \frac{v^{4}}{r^{7}}+c_{12} \frac{v^{6}}{r^{11}}+c_{13} \frac{v^{8}}{r^{15}}+\cdots \\
& +c_{21} \frac{v^{4}}{r^{10}}+c_{22} \frac{v^{6}}{r^{14}}+c_{23} \frac{v^{8}}{r^{18}}+\cdots \\
& +c_{31} \frac{v^{4}}{r^{13}}+c_{32} \frac{v^{6}}{r^{17}}+c_{33} \frac{v^{8}}{r^{21}}+\cdots
\end{aligned} \mid \# \text { loops }
$$

Comparing with the expansion of classical supergravity (4.6) in terms of $v^{2} / r^{7} \Gamma$ we see that those terms match the terms on the diagonal. Terms to left of the diagonal have to vanish. Otherwise $\Gamma$ for large separations - the regime of gravity - those would dominate the behavior of gravity at a given order of $v$. Indeed $\Gamma$ the result $c_{21}=0$ of [BB] was celebrated as strong support for the M(atrix)-Model conjecture. In [PSS1] it was shown that in fact all coefficients $c_{n 1}$ for $n>1$ have to vanish by supersymmetry. A bit later $[$ [BBPT] found

$$
c_{22}=\frac{225}{64}
$$

which agrees with the expectation (4.6) from supergravity. Again $\Gamma$ in [PSS2] $Г$ Paban $Г$ Sethi and Stern were able to deduce from supersymmetry that $c_{n 2}$ vanishes for $n>2$. Up to today there are no results for terms below the diagonal $c_{n m}$ for $m>3$ corresponding to more than six powers of relative velocity. Further non-renormalization theorems are not expected $\Gamma$ there are even indications of infrared problems[DEG] at higher loop orders. Therefore it would be very interesting to see whether M(atrix)-Theory agrees with the gravity prediction of

$$
c_{33}=\frac{16875}{1024} \text {. }
$$

Unfortunately 5 to derive this number from M(atrix)-Theory one would have to calculate about one hundred diagrams even in the background field formalism. The calculation of these three-loop amplitudes involves complicated Schwinger parameter integrals that probably cannot be calculated analytically but only numerically. This makes it plausible that the task of determining $c_{33}$ is only possible with massive computer use.

\subsection{Three-Graviton Scattering at Leading Order}

The investigation of scattering processes of more than two particles allows for much more sophisticated tests of the M(atrix)-Theory conjecture than the two-particle case because the kinematics is much richer: There are not only two parameters $\Gamma$ the impact parameter $b$ and the relative velocity $v$ Гbut there are already nine of them for three-particle processes: From two vectors of relative velocities and two vector impact parameters one can form ten invariants under the $S O(9)$ rotational group $\Gamma$ namely there are the four magnitudes plus 
six relative scalar products. One of them can be removed with the help of time translation invariance or the energy conservation constraint (this is the generalization of the fact that we could assume $\vec{v}$ and $\vec{b}$ to be orthogonal in the two-particle case).

This allows for more terms with different tensor structures in the effective action at a given order of perturbation theory; each of them would have to come out with the correct coefficients.

As the string theory tree level diagram for three D0-particles can be transformed into a two loop gauge theory ribbon diagram $\Gamma$ we expect the $\mathrm{M}$ (atrix)-Model to contain the three particle interactions at its two-loop level.

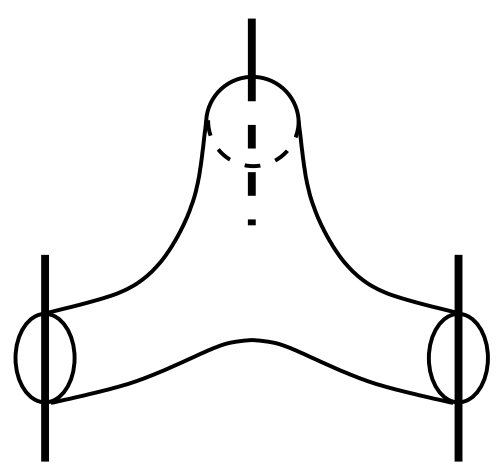

Fig. 11: Three-particle scattering as a string diagram

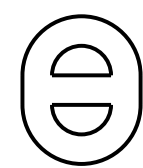

Fig. 12: The M(atrix)-Model three-particle diagram

When $\Gamma$ in fall 1998ГDine and Rajaraman[DR] looked into this problem $\Gamma$ the disagreement they found came as a shock to the community. Their argument roughly went along the following lines: They studied a three-graviton interaction in a limit where the separation $r$ between two particles (say particle one and two) is much smaller than the distance $R$ to the third. They argued that in supergravity of one graviton between particle one and two and one between two and three.

From applying Newton's force law in light cone-coordinates twice Tone expects this process to be proportional to

$$
\frac{\left(v_{1}-v_{2}\right)^{2}\left(v_{2}-v_{3}\right)^{2}\left(v_{3}-v_{1}\right)^{2}}{R^{7} r^{7}} .
$$

The M(atrix)-Theory side of the argument can be understood with the powercounting rules we derived above: As there are two distance scales there will be two loops 


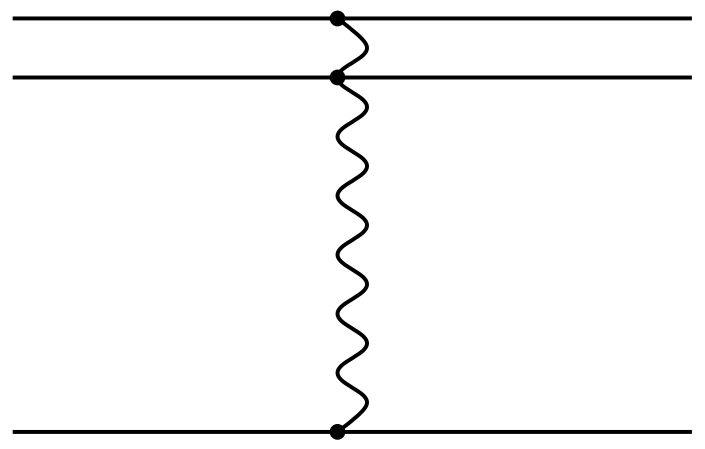

Fig. 13: The supergravity process studied in [DR]

each being regulated by cutting off momenta like $\int_{r}^{\infty} d \omega$ and $\int_{R}^{\infty} d \Omega$. Four external lines (two incoming and two outgoing) should be attached to the large $R$ loop. They have to produce relative velocities between largely separated particles. Thus $\Gamma$ the generic diagram looks like

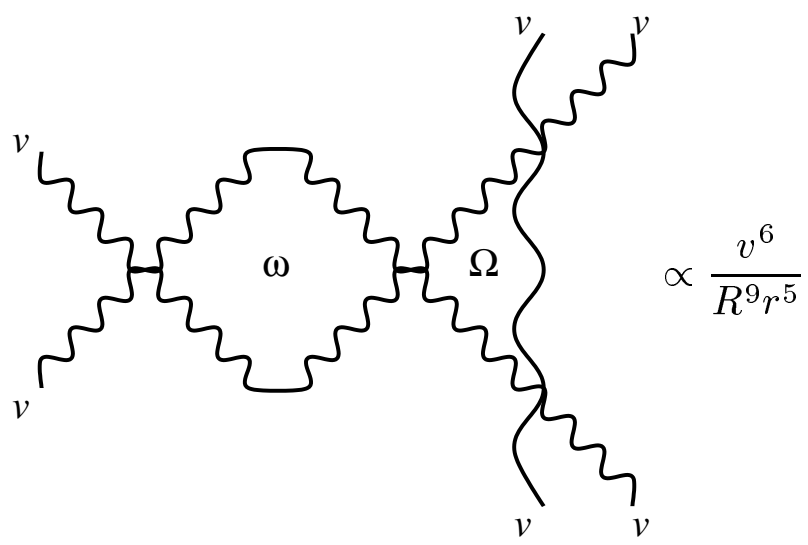

Obviously $\Gamma$ this is the wrong dependence on the two distance scales. The rest of the paper went a long way to carefully calculate the gravity amplitude both from supergravity Feynman rules and from string theory to make sure the coefficient does not vanish.

As it became clear a few months later $\Gamma$ the argument given above contains a flaw. The error is that beyond the one-loop level the background does not only modify the propagators but also the vertices as some of the fields at a vertex might be external. Especially Lusing the background field formalism Tthe M(atrix)-Model also contains effective bosonic three point vertices that allow for diagrams of the "setting sun" type:

This diagram is symmetric with respect to $\omega$ and $\Omega$ and it is therefore not obvious that it cannot contain terms of the needed form

$$
\frac{v^{6}}{r^{7} R^{7}}
$$

In an impressive calculationГOkawa and Yoneya showed that $\Gamma$ to leading order $\Gamma$ the $\mathrm{M}(\mathrm{a}-$ trix)-Model and supergravity indeed give exactly the same amplitude for three graviton processes at leading order. 


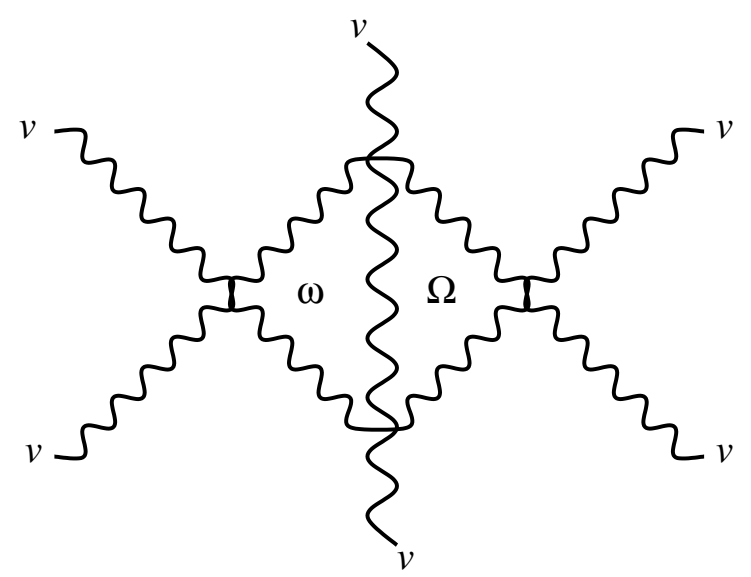

Fig. 14: The setting sun diagram

In their two papers [OY1][OY2] $\Gamma$ the scattering amplitude on the supergravity side was derived much in the same source-probe approach that we used in the previous chapter where to work out the correction to the action of a particle in some ambient space-time that is generated by the source particle. This approach becomes more and more complicated for higher order calculations of more than two particle processes since there is no exact solution for more than one source particle. One has to resort to a perturbative solution in terms of Newton's constant $\kappa$. The book-keeping of the orders of $\kappa$ that are required at the different stages of the calculation and the possibility of recoil effects makes such an approach not straightforward and difficult to extend to even more complicated situations like the ones we are going to consider later in this chapter.

Instead $\Gamma$ as in [HPSW] $\Gamma$ we treat perturbative supergravity as a field theory and apply the formalism of Feynman diagram perturbation theory. This approach has the advantage to be straightforward $\Gamma$ very clear-cut $\Gamma$ and easily extendable $\Gamma$ in particular $\Gamma$ it is well suited to be put on a computer for an computer algebra system like FORM to simplify.

Our normalization of the bosonic part of the Cremmer-Julia-supergravity is

$$
\begin{aligned}
\mathcal{L}=- & \frac{1}{2 \kappa^{2}} \sqrt{-g} \mathcal{R}-\frac{1}{8} \sqrt{-g}\left(F_{M N P Q}\right)^{2} \\
& \quad-\frac{\sqrt{3}}{12^{3} \kappa^{3}} \epsilon^{M_{1} \ldots M_{11}} F_{M_{1} M_{2} M_{3} M_{4}} F_{M_{5} M_{6} M_{7} M_{8}} C_{M_{9} M_{10} M_{11}}
\end{aligned}
$$

where $F_{M N P Q}=4 \partial_{[M} C_{N P Q]} \Gamma g=\operatorname{det} g_{M N} \Gamma$ and capital latin indices from the middle of the alphabet run from 0 to $10 . \kappa$ is Newton's constant in eleven dimensions. As usual in perturbative (quantum) gravity $\Gamma$ we consider small fluctuations $h_{M N}$ around the flat background metric $\eta_{M N}$ Ti.e. $g_{M N}=\eta_{M N}+\kappa h_{M N}$. The harmonic gauge $\partial_{N} h^{N}{ }_{M}-$ $\frac{1}{2} \partial_{M} h^{N}{ }_{N}=0$ (indices are raised and lowered with the flat metric) being covariant is convenient for our purposes. The propagator of the metric fluctuations turns out to be

$$
\left\langle h_{M N}(k) h_{P Q}(-k)\right\rangle=-\frac{i / 2}{k^{2}+i \epsilon}\left(\eta_{M P} \eta_{N Q}+\eta_{M Q} \eta_{N P}-\frac{2}{9} \eta_{M N} \eta_{P Q}\right) .
$$


We want to study three-graviton scattering at tree level. As there are no terms in the supergravity action that are quadratic in the gravitons and linear in one of the other fields (graviphoton or gravitiniГcf. Fig. 13) $\Gamma$ the only contribution comes from the pure gravity sector $\Gamma$ i.e. Tthe Einstein-Hilbert term.

In particular $\Gamma$ we shall need the two and three graviton vertices arising from its expansion. These are quite lengthy expressions that have been calculated in [Sa].

We consider now the elastic scattering process $1+2+3 \rightarrow 1^{\prime}+2^{\prime}+3^{\prime}$ of three gravitons into three gravitons and concentrate only on helicity preserving terms $\Gamma$ i.e. terms in the amplitude proportional to

$$
\left(h_{1} \cdot h_{1}^{\prime}\right)\left(h_{2} \cdot h_{2}^{\prime}\right)\left(h_{3} \cdot h_{3}^{\prime}\right),
$$

$h_{i}$ being the external transversal graviton polarization tensors and $\left(h_{i} \cdot h_{i}^{\prime}\right)=h_{i}^{m n} h_{i}^{\prime m n}$. The eleven-dimensional momenta are conveniently parameterized in a light-cone frame $M=(+,-, m)$ as

$$
p_{i}=\left(-\frac{1}{2}\left(\mathbf{v}_{i}-\frac{\mathbf{q}_{i}}{2}\right)^{2}, 1, \mathbf{v}_{i}-\frac{\mathbf{q}_{i}}{2}\right), \quad p_{i}^{\prime}=\left(-\frac{1}{2}\left(\mathbf{v}_{i}+\frac{\mathbf{q}_{i}}{2}\right)^{2}, 1, \mathbf{v}_{i}+\frac{\mathbf{q}_{i}}{2}\right)
$$

where $p_{i}^{2}=p_{i}^{\prime 2}=0$ and $i=1,2,3$ Cusing a boldface vector notation for the transversal $S O(9)$ indices $m$. Note $\Gamma$ that we are considering only processes with zero compactified $q_{-}$momentum transfer between incoming particles $i$ and outgoing ones $i^{\prime}$ as compactified momentum transfer is a non-perturbative process in our M(atrix)-Theory background. This will be discussed in more detail in the next chapter where we will investigate momentum transferring solutions. For the time being Clet us restrict our attention only to those kinematical setups that do not change the $p_{-}$components of the individual particles.

Conservation of transversal momentum and energy implies

$$
\mathbf{q}_{1}+\mathbf{q}_{2}+\mathbf{q}_{3}=0 \quad \mathbf{v}_{1} \cdot \mathbf{q}_{1}+\mathbf{v}_{2} \cdot \mathbf{q}_{2}+\mathbf{v}_{3} \cdot \mathbf{q}_{3}=0
$$

Moreover $\Gamma$ we will study the amplitude in an eikonal limit. To be precise this means we keep only terms with at least a double $t$-pole $\left(1 /\left(q_{1}^{2} q_{2}^{2}\right)\right.$ and permutations). Terms in which this minimal pole structure is canceled represent contact interactions and cannot reliably computed in the eikonal M(atrix)-Theory framework we present here. Up to permutations $\Gamma$ there are three types of diagrams: The V-type diagram with a four-point vertex as in Fig. 13Гthe Y-type or cartwheel diagram

and the rescattering diagram:

The tedious but straightforward evaluation of these graphs was performed with the help of the computer algebra system FORM[V]. There are three diagrams of V-type yielding

$$
A_{V}=2 \frac{q_{1}^{2}+q_{2}^{2}+q_{3}^{2}}{q_{1}^{2} q_{2}^{2} q_{3}^{2}} v_{12}^{2} v_{23}^{2} v_{31}^{2}+O\left(v^{5} q^{-3}\right),
$$




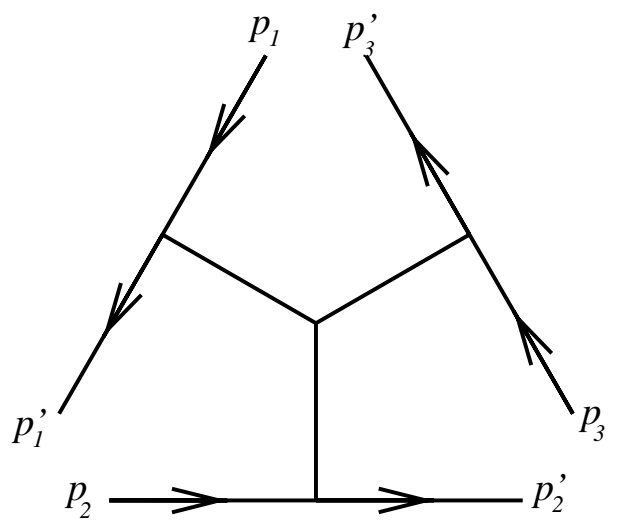

Fig. 15: Y-type diagram

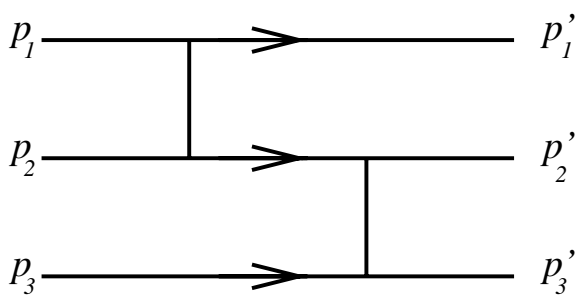

Fig. 16: Rescattering diagram

where we suppress terms higher order in $\mathbf{v}_{i}$ and lower order in $\mathbf{q}_{i}$. Similarly $\Gamma$ there is only one Y-type graph that can be written as follows:

$$
A_{Y}=-\frac{1}{q_{1}^{2} q_{2}^{2} q_{3}^{2}}\left(\left(q_{1}^{2}+q_{2}^{2}+q_{3}^{2}\right) v_{12}^{2} v_{23}^{2} v_{31}^{2}-\Upsilon^{2}\right)+O\left(v^{5} q^{-3}\right)
$$

where we defined

$$
\Upsilon=v_{23}^{2} \mathbf{q}_{2} \cdot \mathbf{v}_{12}+v_{31}^{2} \mathbf{q}_{3} \cdot \mathbf{v}_{23}+v_{12}^{2} \mathbf{q}_{1} \cdot \mathbf{v}_{31} .
$$

Note that $\Gamma$ using $(5.4) \Gamma \Upsilon$ is odd under permutations of the particle labels $12 \Gamma$ and 3 . In particular it is invariant under cyclic permutations. Finally $\mathrm{Twe}$ have contributions of the six re-scattering graphs:

$$
\begin{aligned}
A_{r}=-\frac{1}{q_{1}^{2} q_{2}^{2} q_{3}^{2}}[ & \left(q_{1}^{2}+q_{2}^{2}+q_{3}^{2}\right) v_{12}^{2} v_{23}^{2} v_{31}^{2} \\
& \left.-\left(\left(\frac{q_{1}^{2} v_{12}^{2} v_{31}^{2}}{\mathbf{q}_{2} \cdot \mathbf{v}_{12}}\right) \Upsilon+\frac{1}{8}\left(\frac{q_{1}^{2} v_{12}^{2} v_{31}^{2}}{\mathbf{q}_{2} \cdot \mathbf{v}_{12}}\right)^{2}+\text { cyclic }\right)\right]+O\left(v^{5} q^{-3}\right),
\end{aligned}
$$

where "cyclic" indicates the two cyclic permutations over the labels $1 \Gamma 2 \Gamma$ and 3 . Summing up these three diagrams one obtains the final result for the eikonal three-graviton scattering amplitude (throughout this chapter $\Gamma$ we discard overall coefficients of complete amplitudes):

$$
A_{E H}=\frac{1}{q_{1}^{2} q_{2}^{2} q_{3}^{2}}\left[\Upsilon^{2}+\left(\left(\frac{q_{1}^{2} v_{12}^{2} v_{31}^{2}}{\mathbf{q} 2 \cdot \mathbf{v}_{12}}\right) \Upsilon+\frac{1}{8}\left(\frac{q_{1}^{2} v_{12}^{2} v_{31}^{2}}{\mathbf{q}_{2} \cdot \mathbf{v}_{12}}\right)^{2}+\text { cyclic }\right)\right]+O\left(v^{5} q^{-3}\right)
$$


In the above discussion $\Gamma$ we have deliberately omitted the $N$-dependence because we have complete control on the M(atrix)-Theory side for $N_{i}=1$ only. Anyway they can easily be reintroduced with the net result that (5.6) takes an overall factor $N_{1} N_{2} N_{3} \Gamma$ where $N_{i}$ is the $p_{-}$momentum of the graviton $i$ and where we normalize each external leg with a factor of $1 / \sqrt{N_{i}}$.

It should be noted $\mathrm{ffor}$ example by inspection of the supergravity Feynman diagrams that we have really tested non-linear properties of supergravity and not only the linearized free theory of the spin 2 particle. It is well known that that General Relativity is the minimal Lorentz invariant interacting theory with at most two derivatives in the action that contains an interacting spin two particle $\Gamma$ i.e. reduces to linearized gravity upon linearization. Furthermore $\Gamma$ the supergravity of $[\mathbf{C J}]$ is the only possible supersymmetric extension of General Relativity in eleven dimensions.

Thus Tif one were able to prove quantum Lorentz invariance of the M(atrix)-Model in the large $N$ limit the verification of the non-linear structures that are probed by the three particle processes we consider in this chapter would determine that the M(atrix)-Model really yields supergravity and not a different though similar theory in the low energy limit.

\subsection{Three Gravitons in M(atrix)-Theory}

In this section $\Gamma$ we will present the $\mathrm{M}$ (atrix)-Theory side of the calculation above. Our presentation extends the one in [OY1]. To simplify calculations $\Gamma$ it was assumed there that all relative impact parameters $\mathbf{b}_{i j}=\mathbf{b}_{i}-\mathbf{b}_{j}$ are perpendicular to the relative velocities $\mathbf{v}_{i j}$. As we explained above $\Gamma$ this is imposes no restriction in the case of two particles but substantially restricts the number of $S O(9)$ tensor invariants for the three particle case. To have access to many more independent terms and therefore perform a much more fine grained analysis $\Gamma$ we will here close this gap and treat the most general three particle kinematics.

For this slightly more general setup a mild generalization for the heat-kernel

$$
\left(-\partial_{t}^{2}+r_{i j}(t)^{2}\right) \Delta\left(t, t^{\prime}, \sigma\right)=-\partial_{\sigma} \Delta\left(t, t^{\prime}, \sigma\right)
$$

is needed. Here we wrote $\mathbf{r}_{i j}(t)=\mathbf{b}_{i j}+t \mathbf{v}_{i j}$. The appropriate heat-kernel is

$$
\begin{aligned}
\Delta=\sqrt{\frac{v_{i j}}{2 \pi \sinh \left(2 \sigma v_{i j}\right)}} \exp [ & -v_{i j}^{2} t_{-}^{2} \operatorname{coth}\left(\sigma v_{i j}\right)-\sigma r_{i j}\left(t_{+}\right)^{2} \\
& \left.-\left(\frac{\mathbf{v}_{i j} \cdot \mathbf{r}_{i j}\left(t_{+}\right)}{v_{i j}}\right)^{2} \frac{1}{v_{i j}}\left(\tanh \left(\sigma v_{i j}\right)-\sigma v_{i j}\right)\right]
\end{aligned}
$$

where we have again used $t_{ \pm}=\left(t \pm t^{\prime}\right) / 2$. Note that $t_{+}$appears only thru $\mathbf{r}_{i j}\left(t_{+}\right)$as we would have expected for a local effective Lagrangian. 


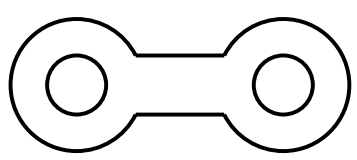

Fig. 17: Dumbbell ribbon diagram

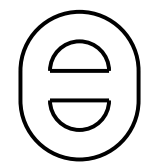

Fig. 18: Setting sun ribbon diagram

There are three different two loop topologies: The dumbbell

the setting sun

and the figure eight.

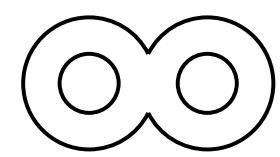

Fig. 19: Figure eight ribbon diagram

The resulting effective actions are denoted by $\Gamma_{o \curvearrowleft} \Gamma_{Y}$ and $\Gamma_{V}$ respectively (actually $\Gamma$ any terms from the setting sun diagram that can be written as a total $d / d \sigma_{i}$ derivative of a polynomial times three propagators are included in $\Gamma_{V}$ rather than $\Gamma_{Y} \Gamma_{\text {see }}$ [OY1] for details). Because we do not perform a Legendre transformation $\Gamma$ we have to include all possible diagrams. As disconnected diagrams contribute only to disconnected diagrams on the supergravity side $\Gamma$ they can be left out of this analysis. But connected one-particle reducible graphs have to be included and correspond to supergravity rescattering processes that were described in [OY2].

The Okawa-Yoneya result may be stated (somewhat implicitly) as the effective action

$$
\Gamma_{2 \text { loop }}=\Gamma_{o \multimap}+\Gamma_{V}+\Gamma_{Y}
$$

The contribution of the dumbbell graphs is

$$
\Gamma_{o \multimap o}=-\frac{1}{2} \sum_{i} \int d t_{1} d t_{2}\left\langle\partial_{t_{1}}^{2} Y_{i i}^{m}\left(t_{1}\right)\right\rangle \Delta\left(t_{1}-t_{2}\right)\left\langle\partial_{t_{2}}^{2} Y_{i i}^{m}\left(t_{2}\right)\right\rangle
$$

with the one loop expectation value

$$
\begin{aligned}
\left\langle\partial_{t}^{2} Y_{i i}^{m}(t)\right\rangle=-32 \sum_{j} \int_{0}^{\infty} d \sigma & r_{i j}^{m}(t) \sinh ^{4}\left(\frac{\sigma v_{i j}}{2}\right) \\
& \left.+\frac{v_{i j}^{m}}{v_{i j}} \cosh \left(\frac{\sigma v_{i j}}{2}\right) \sinh ^{3}\left(\frac{\sigma v_{i j}}{2}\right) \partial_{t}\right] \Delta\left(\sigma, 0, \mathbf{r}_{i j}(t)\right)
\end{aligned}
$$


and $\Delta\left(t_{1}-t_{2}\right)=\int_{0}^{\infty} d \sigma \Delta(\sigma, t, 0)$ is the propagator for a free massless scalar field in one dimension. Furthermore

$$
\begin{aligned}
& \Gamma_{V}=- 128 \sum_{i j k} \int_{-\infty}^{\infty} d t \int_{0}^{\infty} d \sigma_{1} d \sigma_{2} \sinh ^{3}\left(\frac{\sigma_{1} v_{i j}}{2}\right) \sinh ^{3}\left(\frac{\sigma_{2} v_{j k}}{2}\right) \\
& \times {\left[\frac{2 \mathbf{v}_{i j} \cdot \mathbf{v}_{j k}}{v_{i j} v_{j k}} \cosh \left(\frac{\sigma_{1} v_{i j}}{2}\right) \cosh \left(\frac{\sigma_{2} v_{j k}}{2}\right)-\sinh \left(\frac{\sigma_{1} v_{i j}}{2}\right) \sinh \left(\frac{\sigma_{2} v_{j k}}{2}\right)\right] } \\
& \times \Delta\left(\sigma_{1}, 0, \mathbf{r}_{i j}(t)\right) \Delta\left(\sigma_{2}, 0, \mathbf{r}_{j k}(t)\right)
\end{aligned}
$$

along with

$$
\begin{aligned}
\Gamma_{Y}=- & \sum_{i j k} \int d t_{+} d t_{-} \int_{0}^{\infty} d \sigma_{1} d \sigma_{2} d \sigma_{3} \\
& \times P_{Y}\left(\sigma_{1}, \sigma_{2}, \sigma_{3}, \mathbf{r}_{i j}(t), \mathbf{r}_{j k}(t), \mathbf{r}_{k i}(t), \mathbf{v}_{i j}, \mathbf{v}_{j k}, \mathbf{v}_{k i}, t_{-}\right) \\
& \times \Delta\left(\sigma_{1}, t_{-}, \mathbf{r}_{i j}\left(t_{+}\right)\right) \Delta\left(\sigma_{2}, t_{-}, \mathbf{r}_{j k}\left(t_{+}\right)\right) \Delta\left(\sigma_{3}, t_{-}, \mathbf{r}_{k i}\left(t_{+}\right)\right) .
\end{aligned}
$$

The Okawa-Yoneya computation of the function $P_{Y}$ is a very impressive technical achievement and the result is a quadratic polynomial in the variables $\mathbf{r}_{i j}\left(t_{+}\right)$and $t_{-}$(the result itself is given by equation (3.47) of [OY1] along with three pages of the appendices of that work). Its correctness (at least to leading order in $\mathbf{v}_{i j}$ ) is well tested by comparison with supergravity.

A remark on the $N$ dependence of the two-loop effective action $\Gamma_{2-\text { loop }}$ is in order. The planar two-loop graphs trace over three independent $U(N)$ indices $i, j, k$ as they all have three boundary components in the 't Hooft's ribbon notation we have been using above. Thus they give rise to three body interactions as we had expected from the world sheet duality argument given above. For backgrounds consisting of three blocks proportional to unit matrices of size $N_{i}\left(i=1,2,3\right.$ with $\left.\sum_{i} N_{i}=N\right)$ the sums $\sum_{i j k}$ reduce to $N_{1} N_{2} N_{3} \sum_{i j k=1}^{3}$ and $\Gamma_{2-\text { loop }}$ scales homogeneously like $N^{3}$ to all orders in $v_{i j} \Gamma$ precisely like the corresponding supergravity term (5.6).

But as we have seen in the first section of the last chapter Thaving blocks of multiples of unit $N_{i} \times N_{i}$ matrices can be interpreted as $N_{i}$ coinciding particles with possible massless strings stretching in between. The restriction to diagonal matrices therefore not only excludes degrees of freedom with large masses but also massless ones. We would be throwing away light degrees of freedom by hand $\Gamma$ and proceeding like this is not physical. Rather Tone should insert the ground state wave function for the $U\left(N_{1}\right) \mathrm{M}($ atrix $)$-Model $\Gamma$ which is unfortunately not available. Therefore $\Gamma$ to get around these difficulties we will take our result seriously only for $N_{i}=1$.

Up to now $\Gamma$ we have simply restated the results of [OY1]. In what follows $\Gamma$ we will compare these results with the tree level supergravity $S$-matrix and in doing so show how to relax the restriction $\{\mathbf{b} \cdot \mathbf{v}\}=0$. Thereafter $\Gamma$ the same techniques will be employed to 
compare the M(atrix)-Theory prediction for the next to leading order in $\mathbf{v}_{i j}$ with one-loop supergravity.

Let us begin with the most difficult contribution $\Gamma_{Y}$ as in (5.11). One might suspect $\Gamma$ that since the integrand depends on three proper time parameters $\sigma_{1} \Gamma \sigma_{2} \Gamma$ and $\sigma_{3} \Gamma$ the result ought to correspond to the triple pole structure of the Y-type diagrams in supergravity $\Gamma$ and indeed this naïve suspicion will be borne out in the following. As we have done the supergravity calculation in momentum space $\Gamma$ we will perform a Fourier transform to the M(atrix)-Model effective action. The LSZ-formula tells us that the leading-order spinindependent term in the three-particle $S$-matrix is given by

$$
S^{3 \rightarrow 3}=\int d^{9} b_{1} d^{9} b_{2} d^{9} b_{3} e^{i\left(\mathbf{q}_{1} \cdot \mathbf{b}_{1}+\mathbf{q}_{2} \cdot \mathbf{b}_{2}+\mathbf{q}_{3} \cdot \mathbf{b}_{3}\right)} \Gamma_{2-\text { loop }}
$$

Note that we have dropped contributions corresponding to disconnected processes $\Gamma$ so $\Gamma_{2-\text { loop }}$ no longer appears in the exponent. The transverse kinematics described by (5.12) involve initial and final momenta

$$
\begin{aligned}
\mathbf{p}_{i} & =\mathbf{v}_{i}-\frac{\mathbf{q}_{i}}{2} \\
\mathbf{p}_{i}^{\prime} & =\mathbf{v}_{i}+\frac{\mathbf{q}_{i}}{2}
\end{aligned}
$$

in accord with our parameterization (5.3) of the kinematics in the supergravity process. Note that at this stage $\mathbf{v}_{i}$ is not a velocity anymore but rather the average momentum of the $i$ th particle $\Gamma \mathbf{v}_{i}=\left(\mathbf{p}_{i}+\mathbf{p}_{i}^{\prime}\right) / 2$. Since $\Gamma_{2-\text { loop }}$ depends on relative quantities only $\Gamma$ the integral over the average impact parameter $\left(\mathbf{b}_{1}+\mathbf{b}_{2}+\mathbf{b}_{3}\right) / 3$ yields the usual momentum conserving $\delta^{(9)}\left(\mathbf{q}_{1}+\mathbf{q}_{2}+\mathbf{q}_{3}\right)$ which we drop from now on. Concentrating on the $\Gamma_{Y}$ contribution we then have

$$
\begin{aligned}
S_{Y}^{3 \rightarrow 3}=- & \int d^{9} \mathbf{b}_{13} d^{9} \mathbf{b}_{23} \exp \left(i \mathbf{q}_{1} \cdot \mathbf{b}_{13}+i \mathbf{q}_{2} \cdot \mathbf{b}_{23}\right) \int d t_{+} d t_{-} \int_{0}^{\infty} d^{3} \sigma \\
& \times P_{Y}\left(\sigma_{i}, \mathbf{r}_{i j}\left(t_{+}\right), \mathbf{v}_{i j}, t_{-}\right) \\
& \times \Delta\left(\sigma_{1}, t_{-}, \mathbf{r}_{12}\left(t_{+}\right)\right) \Delta\left(\sigma_{2}, t_{-}, \mathbf{r}_{23}\left(t_{+}\right)\right) \Delta\left(\sigma_{3}, t_{-}, \mathbf{r}_{31}\left(t_{+}\right)\right)
\end{aligned}
$$

The leading contribution to three-body scattering should depend on the sixth power of the velocities $\mathbf{v}_{12} \Gamma \mathbf{v}_{23}$ Tand $\mathbf{v}_{31}$ as we expect agreement with the supergravity amplitude. However $\Gamma$ if one examines the polynomial $P_{Y}$ Tits leading behavior is quadratic in velocities and the heat-kernels $\Delta$ are velocity independent to leading order. In order to see explicitly how the cancellations of terms quadratic and quartic in velocities occur $\Gamma$ two observations

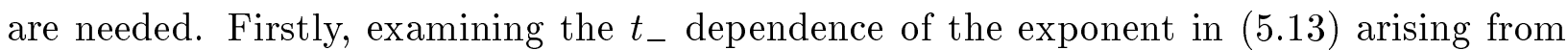
the three heat-kernels

$$
-t_{-}^{2}\left(v_{12}^{2} \operatorname{coth}\left(\sigma_{1} \mathbf{v}_{12}\right)+v_{23}^{2} \operatorname{coth}\left(\sigma_{2} \mathbf{v}_{2} 3\right)+v_{31}^{2} \operatorname{coth}\left(\sigma_{3} \mathbf{v}_{3}\right)\right)=:-t_{-}^{2} P,
$$

one sees that under the Gaussian $t_{-}$integral $\Gamma$ all terms odd in $t_{-}$can be discarded by symmetric integration. As

$$
\frac{\partial}{\partial t_{-}} e^{-P t_{-}^{2}}=-2 P t_{-} e^{-P t_{-}^{2}}
$$


we find after a partial integration

$$
\int_{-\infty}^{\infty} d t_{-} t_{-}^{2 n} e^{-P t_{-}^{2}}=-\int_{-\infty}^{\infty} d t_{-} \frac{1}{2 P} t_{-}^{2 n-1} \frac{\partial}{\partial t_{-}} e^{-P t_{-}^{2}}=\int_{-\infty}^{\infty} d t_{-} \frac{2 n-1}{2 P} t_{-}^{2 n-2} e^{-P t_{-}^{2}}
$$

and by induction

$$
\int_{-\infty}^{\infty} d t_{-} t_{-}^{2 n} e^{-P t_{-}^{2}}=\int_{-\infty}^{\infty} d t_{-} \frac{(2 n-1) ! !}{(2 P)^{n}} e^{-P t_{-}^{2}}
$$

NextTobserve that acting on the three heat-kernels with $d / d t_{+}$brings down a factor

$$
\begin{aligned}
Q:=-2( & \frac{\mathbf{v}_{12} \cdot \mathbf{r}_{12}\left(t_{+}\right)}{v_{12}} \tanh \left(\sigma_{1} v_{12}\right) \\
& \left.\quad+\frac{\mathbf{v}_{23} \cdot \mathbf{r}_{23}\left(t_{+}\right)}{v_{23}} \tanh \left(\sigma_{2} v_{23}\right)+\frac{\mathbf{v}_{31} \cdot \mathbf{r}_{31}\left(t_{+}\right)}{v_{31}} \tanh \left(\sigma_{3} v_{31}\right)\right) .
\end{aligned}
$$

The second observation is that every occurrence of the factor $\left(\sigma_{1} \mathbf{r}_{12} \cdot \mathbf{v}_{12}\right)$ in $P_{Y}$ is actually part of a factor $Q$ and can be completed as such. Therefore $\Gamma$ writing $Q$ as $d / d t_{+}$acting on the $\Delta$ 's and subsequently integrating by parts removes all dependence on $\mathbf{r}_{12} \Gamma \mathbf{r}_{23}$ and $\mathbf{r}_{31}$. Coupled with the first observation $\Gamma$ one in fact finds that miraculously all terms proportional to squares and the fourth order of velocity cancel. We stress that no restriction involving inner products of velocities and impact parameters needs to be imposed for this cancellation to take place.

It is now advantageous to interchange the $d t_{+}$and $d^{9} \mathbf{b}$ integrals and thereafter shift the integration variable $\mathbf{b}_{13} \mapsto \mathbf{r}_{13}\left(t_{+}\right)$along with $\mathbf{b}_{23} \mapsto \mathbf{r}_{23}\left(t_{+}\right)$so that the $t_{+}$integral may be performed thus yielding an energy conserving delta function

$$
\begin{aligned}
S_{Y}^{3 \rightarrow 3}=- & (2 \pi) \delta\left(\mathbf{q}_{1} \cdot \mathbf{v}_{13}+\mathbf{q}_{2} \cdot \mathbf{v}_{23}\right) \\
& \times \int d^{9} \mathbf{r}_{13} d^{9} \mathbf{r}_{23} \exp \left(i \mathbf{q}_{1} \cdot \mathbf{r}_{13}+i \mathbf{q}_{2} \cdot \mathbf{r}_{23}\right) \int_{-\infty}^{\infty} d t_{-} \int_{0}^{\infty} d^{3} \sigma \\
& \times \tilde{P}_{Y}\left(\sigma_{i}, \mathbf{r}_{i j}, \mathbf{v}_{i j}\right) \Delta\left(\sigma_{1}, t_{-}, \mathbf{r}_{12}\right) \Delta\left(\sigma_{2}, t_{-}, \mathbf{r}_{23}\right) \Delta\left(\sigma_{3}, t_{-}, \mathbf{r}_{31}\right)
\end{aligned}
$$

where the tilde over $P_{Y}$ indicates that we have performed the manipulations indicated in the two observations above.

So far $\Gamma$ we have managed to rewrite the $\Gamma_{Y}$ contribution to the M(atrix)-Theory $S$ matrix as (suppressing from now on the energy preserving delta function $(2 \pi) \delta\left(\mathbf{q}_{1} \cdot \mathbf{v}_{13}+\right.$ $\left.\left.\mathbf{q}_{2} \cdot \mathbf{v}_{23}\right)\right)$

$$
\begin{aligned}
S_{Y}^{3 \rightarrow 3}=- & \int d^{9} \mathbf{r}_{13} d^{9} \mathbf{r}_{23} \exp \left(i \mathbf{q}_{1} \cdot \mathbf{r}_{13}+i \mathbf{q}_{2} \cdot \mathbf{r}_{23}\right) \int_{0}^{\infty} d^{3} \sigma \frac{1}{\sqrt{4 \pi P}} \\
& \times\left(\tilde{P}_{Y}+\tilde{P}_{Y}^{m} r^{m}+r^{m} \tilde{P}_{Y}^{m n} r^{n}\right) \Delta\left(\sigma_{1}, 0, \mathbf{r}_{12}\right) \Delta\left(\sigma_{2}, 0, \mathbf{r}_{23}\right) \Delta\left(\sigma_{3}, 0, \mathbf{r}_{31}\right) .
\end{aligned}
$$


Note that we have performed the integral over $t_{-}$as explained above. Furthermore $\Gamma \tilde{P}_{Y} \Gamma \tilde{P}_{Y}^{m}$ and $\tilde{P}_{Y}^{m n}$ are functions of the $\sigma_{i}$ and $\mathbf{v}_{i j}$ only $(i j=(12,23,31))$ and their leading behavior goes with the sixth power of velocity. Also $\Gamma$ their coupling to $\mathbf{r}_{i j}$ has been schematized.

We proceed by interchanging the Fourier integrals over $\mathbf{r}_{13}$ and $\mathbf{r}_{23}$ with those over proper time parameters $\sigma_{i}$. If $\Gamma$ for the moment $\Gamma$ we content ourselves with the leading order in velocities $\Gamma$ the $\mathbf{r}$ dependence of the exponent of (5.14) reads

$$
\exp \left(i \mathbf{q}_{1} \cdot \mathbf{r}_{13}+i \mathbf{q}_{2} \cdot \mathbf{r}_{23}-r_{A}^{m} \mathcal{O}_{A B} r_{B}^{m}\right), \quad \mathcal{O}=\left(\begin{array}{cc}
\sigma_{1}+\sigma_{3} & -\sigma_{1} \\
-\sigma_{1} & \sigma_{1}+\sigma_{2}
\end{array}\right)
$$

where the index $A=(13,23)$. The matrix $\mathcal{O}$ has determinant $p=\sigma_{1} \sigma_{2}+\sigma_{2} \sigma_{3}+\sigma_{3} \sigma_{1} \Gamma$ and the Gaussian integral may be performed after we have rewritten the exponent in diagonal form $\Gamma$

$$
\begin{aligned}
\exp \left[-\left(\sigma_{1}+\sigma_{3}\right)\right. & \left(\mathbf{r}_{13}-\frac{i \mathbf{q}_{1}+2 \sigma_{1} \mathbf{r}_{23}}{2\left(\sigma_{1}+\sigma_{3}\right)}\right)^{2} \\
& \left.\quad-\frac{p}{\sigma_{1}+\sigma_{3}}\left(\mathbf{r}_{23}-\frac{i\left(\left(\sigma_{1}+\sigma_{3}\right) \mathbf{q}_{2}+\sigma_{1} \mathbf{q}_{1}\right)}{2 p}\right)^{2}-\frac{\mathbf{q}_{A} \mathcal{O}_{A B}^{-1} \mathbf{q}_{B}}{4}\right]
\end{aligned}
$$

yielding a factor $p^{9}$ after the appropriate shifts in the integration variables. Remarkably $\Gamma$ we find that all terms not proportional to inner products of momentum transfers $\mathbf{q}_{i}$ and velocities $\mathbf{v}_{i j}$ cancel among themselves $\Gamma$ and to leading order in velocities we are left with

$$
S_{Y}^{3 \rightarrow 3}=\frac{\pi^{8}}{4} \int_{0}^{\infty} d^{3} \sigma \frac{1}{p^{3}} \exp \left(-\frac{1}{4 p}\left(q_{1}^{2} \sigma_{2}+q_{2}^{2} \sigma_{3}+q_{3}^{2} \sigma^{1}\right)\right) \Upsilon^{2}
$$

where $\Upsilon$ coincides with the quantity defined in (5.5). FinallyГdoing the $d^{3} \sigma$ integral yields our result

$$
S_{Y}^{3 \rightarrow 3}=32 \pi^{9} \frac{\Upsilon^{2}}{q_{1}^{2} q_{2}^{2} q_{3}^{2}} .
$$

Although we postpone the orchestration of the two-loop leading-velocity M(atrix)-Theory result to the end of this section $\Gamma$ we remark already that (5.15) has precisely the correct form to match with tree-level supergravity graphs of the Y-type in the triple pole sector.

Compared to the $\Gamma_{Y}$ contribution $\Gamma$ the computation of the $S$-matrix elements arising from the $\Gamma_{V}$ terms are very straight forward. By inspection $\Gamma$ the leading contribution from $\Gamma_{V}$ as given in (5.10) is seen to be sixth order in velocity. Hence interchanging $d t_{+}$and $d^{9} \mathbf{b}$ integrals as above and performing the Fourier transforms and proper time $\sigma_{i}$ integrations afterwards $\Gamma$ we find

$$
S_{V}^{3 \rightarrow 3}=-64 \pi^{9} \frac{v_{12}^{2} v_{31}^{2} \mathbf{v}_{12} \cdot \mathbf{v}_{31}}{q_{2}^{2} q_{3}^{2}}+\text { cyclic },
$$

where delta functions over energy and momentum have been suppressed. We emphasize that this result mixes with terms arising from dumbbell graphs $\Gamma_{o \multimap}$ which we will consider 
next. Thus a comparison to supergravity is not possible until we consider the sum of all $\mathrm{M}$ (atrix)-Theory Feynman diagrams which has been the source of some confusion in the literature [DR][DEG].

The final M(atrix)-Theory contribution to the leading order $3 \rightarrow 3 S$-matrix is given by the dumbbell diagrams. In [OY2] $\Gamma$ it has been shown that these graphs can be given the interpretation of recoil corrections to a source probe approximation as we have presented it in section 3.3. In Feynman diagram language there is $\Gamma$ of course $\Gamma$ no artificial distinction between recoil and non-recoil terms (physically since one finds that $\Gamma_{V}$ and $\Gamma_{o \multimap}$ contributions mix $\Gamma$ this is certainly the case).

In order to extract the $S$-matrix contribution from $\Gamma_{o \multimap o}$ as given in (5.8) and (5.9) we begin by rewriting the massless propagator for a scalar field in one dimension as

$$
\Delta\left(t_{1}-t_{2}\right)=\int \frac{d \omega}{2 \pi} \frac{e^{-i \omega\left(t_{1}-t_{2}\right)}}{\omega^{2}+i \epsilon} .
$$

The explicit time derivatives appearing in the truncated tadpoles in (5.9) may be converted into $\omega$ 's upon integration by parts. Then $\Gamma$ in the same fashion as explained above $\Gamma$ interchanging $d^{9} \mathbf{b}$ and time integrals and shifting $\mathbf{b} \mapsto \mathbf{r}(t) \Gamma$ then performing the resulting Fourier transforms and proper time integrals $\Gamma$ we find

$$
\begin{aligned}
S_{o \multimap}^{3 \rightarrow 3}=\pi^{7} & \sum_{i \neq j \neq k} \int d t_{1} d t_{2} \int d \omega \exp \left(-i \mathbf{q}_{j} \cdot \mathbf{v}_{i j} t_{1}-i \omega\left(t_{1}-t_{2}\right)-i \mathbf{q}_{k} \cdot \mathbf{v}_{k i} t_{2}\right) \\
& \times \frac{1}{\omega^{2}+i \epsilon} \frac{v_{i j}^{2} v_{k i}^{2}}{q_{j}^{2} q_{k}^{2}}\left(\mathbf{q}_{j} v_{i j}^{2}-4 \omega \mathbf{v}_{i j}\right) \cdot\left(\mathbf{q}_{k} v_{k i}^{2}-4 \omega \mathbf{v}_{k i}\right) .
\end{aligned}
$$

Note that we have kept only the leading order velocity dependence and discarded those terms in the sum over $U(N)$ indices $i \Gamma j \Gamma$ and $k$ for which the inner matrix index loop running around each end of the dumbbell takes the same value since one may convince oneself that these terms can only lead to disconnected processes. Now $\Gamma$ the integral over $t_{-}=\left(t_{2}-t_{1}\right) / 2$ yields $\delta\left(2 \omega+\mathbf{q}_{j} \cdot \mathbf{v}_{j k}-\mathbf{q}_{k} \cdot \mathbf{v}_{k i}\right)$ and the $t_{+}=\left(t_{1}+t_{2}\right) / 2$ integral yields the usual energy conserving delta function which we suppress as before. The integral over $\omega$ is then trivial and gives the final result

$$
S_{\circ \multimap}^{3 \rightarrow 3}=4 \pi^{9}\left(16 \frac{v_{12}^{2} v_{31}^{2} \mathbf{v}_{12} \cdot \mathbf{v}_{31}}{q_{2}^{2} q_{3}^{2}}+8 \Upsilon \frac{v_{12}^{2} v_{31}^{2}}{q_{2}^{2} q_{3}^{2} \mathbf{q}_{2} \cdot \mathbf{v}_{12}}+\frac{v_{12}^{4} v_{31}^{4} q_{1}^{2}}{q_{2}^{2} q_{3}^{2}\left(\mathbf{q}_{2} \cdot \mathbf{v}_{12}\right)^{2}}+\text { cyclic }\right) .
$$

Observe in particular that here the first term and its permutations exactly cancel the contribution from $S_{V}^{3 \rightarrow 3}$ in (5.16). Clearly then $\Gamma$ one sees that from a physical point of view the split into recoil and non-recoil terms is an artefact of one's approximation scheme. In a Feynman graph approach $\Gamma$ where one simply computes all terms contributing to a given order in velocity all Feynman diagrams on the M(atrix)-Model side. 
In summary the sum $S_{Y}^{3 \rightarrow 3}+S_{V}^{3 \rightarrow 3}+S_{o \multimap}^{3 \rightarrow 3}$ of all terms from equations $(5.15) \Gamma$ (5.16) Tand (5.17) reproduces the tree level supergravity result (5.6). No restriction upon impact parameters or velocities has been imposed in this comparison $\Gamma$ and the present result represents the completion of the leading order spin-independent three-graviton scattering problem whose tortuous history may be followed in the sequence of articles $[\mathbf{D R}] \Gamma[\mathbf{F F}] \Gamma[\mathbf{T} \mathbf{v R}] \Gamma$ and [OY1].

\subsection{Quantum Corrections to Gravity}

In all calculations we have described so far $\Gamma$ we have compared quantum loop calculations on the M(atrix)-Model or gauge theory side with tree level calculations on the supergravity side. As $\hbar$ is the loop counting parameter $\Gamma$ tree level amplitudes come with $\hbar^{0}$ and are therefore classical. This means that $\mathrm{up}$ to now $\Gamma$ we have dealt with classical supergravity. But the ultimate goal is the quantization of gravity and therefore it is a pressing need to obtain results beyond tree level. It is well known that eleven-dimensional gravity is non-renormalizable and $\Gamma$ even for supergravity $\Gamma$ divergencies leading to new terms in the Lagrangian are expected already at one loop level (this is different from supergravity in four dimensions where the first possible counter-term is a three loop term[GGRS]).

Since supergravity is supposed to arise as an effective theory only in the low energy limit the non-renormalizability is not a true problem as it is expected that in the end M-Theory will take care of the high energy degrees of freedom that seem to cause the divergencies. This is the same situation as in ten dimensions where string theory can be viewed as a regulator for supergravity. For the time being $\Gamma$ it is most convenient to think of the divergencies as being regulated by a cut-off $\Lambda_{d}$ in $d$ dimensions. It is natural to take

$$
\Lambda_{11} \propto \ell_{p}^{(11)} \propto \sqrt{\alpha^{\prime}} g_{s}^{\frac{1}{3}}
$$

where have used the M-Theory-string theory dictionary (2.5) of chapter 2. Following [RT] we can assume a $L$-loop term in the gravity effective action to be of the form

$$
\mathcal{S}^{(L)}=\kappa_{11}^{2(L-1)} \sum \Lambda_{11}^{n}\left(\log \Lambda_{11}\right)^{l} \int d^{11} x \sqrt{-g} \mathcal{R}^{m}
$$

where we have used $\mathcal{R}^{m}$ to indicate all scalars built out of the curvature and its covariant derivatives of mass dimension $2 m$. On dimensional grounds $\Gamma$ one finds

$$
n+2 m=9(L-1)+11 .
$$

It has been shown in [FT] that $m>3$ at the one loop order. This can also be seen as follows: M-Theory is to be the strong coupling version of type IIA string theory. Therefore we can expect the terms to scale like $g_{s}^{p}$ for large coupling. If we express $\Lambda_{11}$ in string theory units as above $\Gamma$ we see that $p=n / 3$. In order for $p$ to be an integer $\Gamma n$ has to be a multiple of three. Thus we find

$$
m \equiv 1 \quad(\bmod 3)
$$


i.e. we have terms $\mathcal{R} \Gamma \mathcal{R}^{4} \Gamma \mathcal{R}^{7} \Gamma \ldots$ The $\mathcal{R}^{4}$ term comes with $n=3$ and thus can obviously arise only at one-loop level in string theory. If it came from higher genus diagrams $\Gamma$ the higher power of $g_{s}$ would suppress it at large $g_{s}$ which corresponds to uncompactified MTheory. Our first approach to this term will therefore be a one-loop calculation in IIA string theory[GSW].

This calculation is most easily performed in the Green-Schwarz light-cone formalism where the superstring action is given by

$$
\mathcal{S}_{\text {l.c. }}=-\frac{1}{2} \int d^{2} \sigma\left(T \partial_{\alpha} X^{i} \partial^{\alpha} X^{i}-\frac{i}{\pi} \bar{S}^{a} \rho^{\alpha} \partial_{\alpha} S^{a}\right)
$$

where $S^{a}$ is a two component world sheet Majorana spinor and $\rho^{\alpha}$ are two-dimensional Dirac matrices. The modes of the fermions obey

$$
\left\{S_{m}^{a}, S_{n}^{b}\right\}=\delta^{a b} \delta_{m+n}
$$

which implies that the zero modes form a Clifford algebra and can be represented (using triality) by

$$
S_{0}^{a} \propto\left(\begin{array}{cc}
0 & \gamma_{i \dot{a}}^{a} \\
\gamma_{\dot{a} i}^{a} & 0
\end{array}\right)
$$

with the $S O(8)$ Dirac matrices we have used before $\Gamma$ now acting as $\gamma^{a}: \mathbf{8}_{\mathbf{c}} \rightarrow \mathbf{8}_{\mathbf{v}}$. We represent elements from $\mathbf{8}_{\mathbf{c}}$ by $|\dot{a}\rangle$ and elements of $\mathbf{8}_{\mathbf{v}}$ by $|i\rangle$.

From the $S^{a}$ we can form

$$
R_{0}^{i j}=\frac{1}{4} \gamma_{a b}^{i j} S_{0}^{a} S_{0}^{b}
$$

that act as angular momentum generators as

$$
R_{0}^{i j}|k\rangle=\delta^{j k}|j\rangle-\delta^{i k}|j\rangle
$$

and

$$
R_{0}^{i j}|\dot{a}\rangle=-\frac{1}{2} \gamma_{\dot{a} \dot{b}}^{i j}|\dot{b}\rangle
$$

We will choose our coordinate system in such a way that there is no momentum in the light-cone direction for all four particles. The graviton vertex operator is then given by a product of left- and right-moving bosonic vertex operators which are known from type I string theory:

$$
V_{B}(\zeta, k)=\zeta^{i}\left(\dot{X}^{i}-\frac{1}{4} \bar{S} \gamma^{i j} S k^{j}\right) e^{i k \cdot X} .
$$

The integral that we have to perform to in order to calculate the one-loop amplitude involves a super trace over the fermionic zero mode space $\mathbf{8}_{\mathbf{v}} \oplus \mathbf{8}_{\mathbf{c}}$ :

$$
\operatorname{str}(A)=\langle i|A| i\rangle-\langle\dot{a}|A| \dot{a}\rangle
$$


After some algebra Tone finds that traces of fewer than four powers of $R_{0}$ vanish and that the first non-vanishing term is

$$
t^{i j k l m n p q}=\operatorname{str}\left(R_{0}^{i j} R_{0}^{k l} R_{0}^{m n} R_{0}^{p q}\right) .
$$

After some more algebra This is found to be given by the following lengthy expression:

$$
\begin{aligned}
t^{i j k l m n p q}=- & \frac{1}{2} \epsilon^{i j k l m n p q} \\
-\frac{1}{2} & \left(\left(\delta^{i k} \delta^{j l}-\delta^{i l} \delta^{j k}\right)\left(\delta^{m p} \delta^{n q}-\delta^{m q} \delta^{n p}\right)\right. \\
& +\left(\delta^{k m} \delta^{l n}-\delta^{k n} \delta^{l m}\right)\left(\delta^{p i} \delta^{q j}-\delta^{q j} \delta^{q i}\right) \\
& \left.+\left(\delta^{i m} \delta^{j n}-\delta^{i n} \delta^{j m}\right)\left(\delta^{k p} \delta^{l q}-\delta^{k q} \delta^{l p}\right)\right) \\
+\frac{1}{2}( & \delta^{i k} \delta^{l m} \delta^{n p} \delta^{q i}+\delta^{j m} \delta^{n k} \delta^{l p} \delta^{q i}+\delta^{j m} \delta^{n p} \delta^{q k} \delta^{l i} \\
& +45 \text { terms obtained by antisymmetrizing on each pair of indices }) .
\end{aligned}
$$

These four powers of $R_{0}$ have to be supplied by vertex operators. Since a bosonic vertex operator supplies at most one $R_{0}$ we need at least four external states. As this argument applies in both the left and the right moving sector we will have at least four external gravitons (a graviton is built from a left moving boson times a right moving boson). There are no one-loop contributions with less than four gravitons. This is consistent with our argument above that we do not expect any $\mathcal{R}^{2}$ or $\mathcal{R}^{3}$ terms.

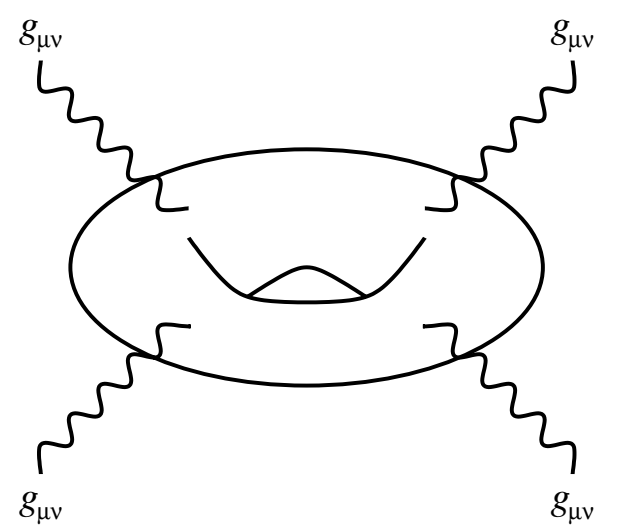

Fig. 20: The loop diagram that yields $\mathcal{R}^{4}$

The zero mode part of the $S$ fields in (5.18) in the factor in front of the exponential is needed to give a non-vanishing contribution in the supertrace as we just explained. Therefore $\Gamma$ for four gravitons $\Gamma$ the prefactors are used up completely $\Gamma$ and up to kinetic factor

$$
K=t^{i_{1} \cdots i_{8}} t^{j_{1} \cdots j_{8}} \zeta_{i_{1} j_{1}}^{(1)} k_{i_{2}}^{(1)} k_{j_{2}}^{(1)} \cdots \zeta_{i_{7} j_{7}}^{(4)} k_{i_{8}}^{(4)} k_{j_{8}}^{(4)}
$$


(we have indicated the polarization of particle $a$ by $\zeta^{(a)}$ and its momentum by $k^{(a)}$ ) we are left with a simple

$$
\left\langle\frac{1}{\Delta}: e^{-i k^{(1)} \cdot X}: \frac{1}{\Delta}: e^{-i k^{(2)} \cdot X}: \frac{1}{\Delta}: e^{-i k^{(3)} \cdot X}: \frac{1}{\Delta}: e^{-i k^{(4)} \cdot X}:\right\rangle
$$

The contribution to the propagators from the non-zero modes of the $S$ 's is

$$
\operatorname{Tr}\left(\prod_{a=1}^{8} \prod_{n=1}^{\infty} w^{n S_{-n}^{a} S_{n}^{a}}\right)=\prod_{n=1}^{\infty}\left(1-w^{n}\right)^{8}=f(w)^{8}
$$

because each fermion level can either be empty or occupied. This exactly cancels the bosonic contribution

$$
\operatorname{Tr}\left(\prod_{i=1}^{8} \prod_{n=1}^{\infty} w^{n \alpha_{-n}^{i} \alpha_{n}^{i}}\right)=f(w)^{-8} .
$$

Like in the bosonic string calculation the integral over the world sheet moduli is unity to leading order in $\alpha^{\prime}$ [GSB][ST] and the total amplitude is given by the kinematical factor $K$ up to a number. $K$ contains two factors of momentum for each graviton. In position representation $\Gamma$ the momenta act as derivatives on the graviton fields and turn them into Riemann tensors. Therefore the covariantization of the amplitude is given by

$$
A=t^{i_{1} \cdots i_{8}} t_{j_{1} \cdots j_{8}} R_{i_{1} i_{2}}^{j_{1} j_{2}} \cdots R_{i_{7} i_{8}}^{j_{7} j_{8}}
$$

Although we have performed a genuine string theory calculation we can think of it as a supergravity calculation in which string theory was merely used as a sophisticated regularization scheme. This can be seen if we restore the appropriate factors of $\alpha^{\prime}$ or the string length $l_{s}$ : The $\mathcal{R}^{4}$ comes with a relative $g_{s}^{2} \alpha^{\prime 3}$ (a quantity that is not available in pure gravity) with respect to the classical Einstein-Hilbert action. The original amplitude in supergravity diverges but string theory provides a finite coefficient for the counter term.

So far $\Gamma$ we have focused on the ten-dimensional case because there $\Gamma$ we have string theory at hand as a tool for computing supergravity amplitudes. Next $\Gamma$ we would like to argue that this $\mathcal{R}^{4}$ counter term is also present in eleven dimensions. There $\mathrm{Tone}$ can do a similar calculation as we have done before [GGV]: These authors consider a loop of a superparticle in a supergravity background. The action of the Green-Schwarz superparticle is

$$
\mathcal{S}_{\text {particle }}=\frac{1}{2} \int d \tau G_{\mu \nu}\left(\dot{X}^{\mu}-i \bar{\Theta} \Gamma^{\mu} \dot{\Theta}\right)\left(\dot{X}^{\nu}-i \bar{\Theta} \Gamma^{\nu} \dot{\Theta}\right)
$$

Just as in the string calculation one can insert eleven-dimensional vertex operators in the world line to obtain scattering amplitudes. The vertex operator for a graviton of polarization $\zeta$ that is inserted at proper time $\tau$ has the form

$$
V(\zeta, \tau)=\zeta^{i k}\left(\dot{X}^{i}-\frac{1}{4 p^{+}} \theta \gamma^{i j} \theta k_{j}\right)\left(\dot{X}^{k}-\frac{1}{4 p^{+}} \theta \gamma^{k l} \theta k_{l}\right) e^{i k \cdot X}
$$


The four graviton amplitude is given by (compare the string discussion above)

$$
A=\frac{1}{\pi^{5 / 2}} \operatorname{Tr} \int d^{11} \mathbf{p} \int_{0}^{\infty} \frac{d \tau}{\tau}\left(\prod_{r=1}^{4} \int_{o}^{\tau} d \tau^{(r)} V^{(r)}\right) e^{-\tau \mathbf{p}^{2}}
$$

By the same argument as above $\mathrm{th}$ the analysis of the fermionic trace leads to the kinematical factor $K$ from (5.19) that finally provides us with the expected tensor structure of the $\mathcal{R}^{4}$ term. In the leading order that we are interested in $\Gamma$ we can replace the $\prod_{r=1}^{4} \int d \tau^{(r)}$ by $\tau^{4}$ and perform the momentum integration. The proper-time integral is then

$$
\int_{0}^{\infty} \frac{d \tau}{\tau} \tau^{4} \tau^{-11 / 2}=\left.\tau^{-3 / 2}\right|_{0} ^{\infty}
$$

As $\tau$ has mass dimension -2 this is just the cubic divergence that we were looking for.

The amplitude is diverging in supergravity and we do not have string theory at our disposal to regulate it. But we would expect M-Theory to play this rôle in eleven dimensions. A non-renormalizable interaction is always a sign for a lack of proper understanding of the high energy degrees of freedom in a quantum theory. Here $\Gamma$ the conjecture is that M-Theory could be used as a definition of this high energy behavior. If the M(atrix)-Model-conjecture is true that $\mathrm{M}$ (atrix)-Model should be able to provide this regularization.

In the rest of this chapter $\Gamma$ we will test this assertion. We will approach this calculation by treating the $\mathcal{R}^{4}$ term as an additional interaction in the supergravity action with an unspecified coefficient $\Gamma$ work out its contribution to scattering amplitudes and try to reproduce the result in a $\mathrm{M}$ (atrix)-Theory calculation. NaïvelyCone would a consider a process with two ingoing and two outgoing particles $\Gamma$ as the $\mathcal{R}^{4}$ term is quartic in gravitons. But in this kinematical setup $\Gamma$ the interaction induced by the new term is a contact interaction.

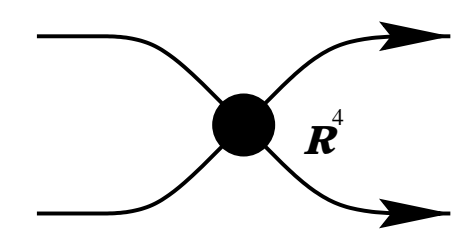

Fig. 21: $\mathcal{R}^{4}$ as a contact interaction

Unfortunately the M(atrix)-Model scattering technology as we have described it so far is not able to handle such kinematics with finite momentum transfer. Remember that in order to calculate scattering amplitudes in M(atrix)-Theory we have always used the diagonal classical background that describes free particles at the classical level. The vectors $\mathbf{b}_{a}$ and $\mathbf{v}_{a}$ that we used to set up the kinematics are defined with respect to this classical solution. As it is $\Gamma$ this classical solution allows only to consider perturbations of the free motion of particles. In the language of scattering theory this means that only 
$t$-channel amplitudes can be obtained from this classical solution. $\mathrm{M}$ (atrix)-Theory in this background provides us only with the leading $t$-pole behavior where $t$ is the square of the momentum transfer. This kinematical restriction has also been described as eikonal scattering.

Thus $\Gamma$ we have two possibilities: Either we find a different classical solution of the $\mathrm{M}$ (atrix)-Model equations of motion or we consider a process in supergravity that is also eikonal but contains the new $\mathcal{R}^{4}$ vertex. We will investigate the first possibility in a later chapter and follow the second here as it was done in[HPSW]. The process we will consider is again a three-particle one wwith a diagram that is of $\mathrm{V}$-type in the classification we have used above. However $\mathrm{Tit}$ differs from the diagram we considered above in that we now take the new $\mathcal{R}^{4}$ vertex as the four-point vertex in the middle:

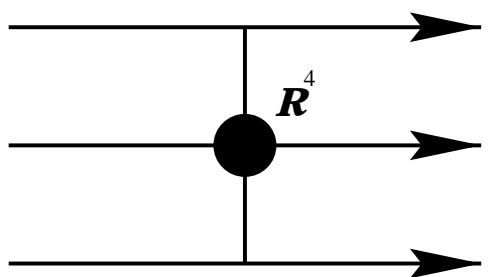

Fig. 22: The eikonal supergravity diagram containing the $\mathcal{R}^{4}$ vertex

Any other possible contribution Tinvolving for instance the Y-type or re-scatteringtype graphs $\Gamma$ will either be sub-dominant in a small velocity and momentum transfer expansion or outside the eikonal kinematical regime of particles that only exchange virtual quanta in the $t$-channel. We then need to compute only one tree level graph with the insertion of the $\mathcal{R}^{4}$ term $\Gamma$ up to permutations of the external legs. This is most easily done by noticing that the linearized tensorial structure appearing in (5.20) is precisely the same as that obtained by computing four-graviton tree-level scattering in a theory of pure gravity in any space-time dimension $[\mathrm{Sa}]$.

One might think that inserting the new vertex in the diagram might lead to incorrect results since the $\mathcal{R}^{4}$ vertex was calculated under the assumption of all four legs being onshell whereas in the diagram we consider $\Gamma$ two of the legs represent virtual particles that might be off-shell. Here $\Gamma$ the key observation is that in the eikonal and spin-less limit (where one discards terms canceling the double pole as well as contractions of momenta with polarizations) the two a priori off-shell legs entering the $\mathcal{R}^{4}$ vertex are effectively put on-shell.

By using the results of [Sa] the computation of the graph in Fig. 22 is then greatly simplified. We find that the result for the part of the amplitude with the external polarizations contracted as in (5.2) and in the kinematical parameterization (5.3) $\Gamma$ can be written as follows (neglecting an overall coefficient):

$$
A_{\mathcal{R}^{4}}=\left(\frac{1}{q_{1}^{2} q_{3}^{2}}\left(\frac{1}{4} v_{12}^{2} v_{23}^{2} q_{2}^{2}+\Upsilon\left(\mathbf{q}_{1} \cdot \mathbf{v}_{12}\right)\right)^{2}+\text { cyclic }\right),
$$


where $\Upsilon$ was defined in (5.5).

Here $\Gamma$ an additional remark is needed. As we have explained earlier $\Gamma$ the $M($ atrix)Model is supposed to arise upon the compactification of eleven-dimensional supergravity on a light-like circle of radius $R_{11}$. Therefore $\Gamma$ the above calculation should have done also after performing this compactification. This would have resulted in terms with different dependences on $R_{11}$. Our philosophy is to find that part of the amplitude that can be reproduced by the $\mathrm{M}$ (atrix)-Theory calculation we are heading on next. Therefore $\Gamma$ we have only picked up those terms that have the correct scaling behavior to have a chance to be found in the following two-loop calculation.

The $N$ dependence of (5.21) that we omitted $\Gamma$ is easily computed to be globally of order $N^{5}$ Tin disagreement with the $N^{3}$ dependence arising at two loops in M(atrix)Theory. This produces indeed the disagreement found in [KVK1]. As we have explained earlier $\Gamma$ we do not consider this as a problem here since we can trust our M(atrix)-Theory result only for $N=3$ Canyway. Thus $\Gamma$ the different scalings with $N$ would maximally show up as an overall numerical factor.

Armed with the above clear-cut scheme for the computation of the M(atrix)-Theory $S$-matrix elements and given the precise agreement of the tree-level supergravity amplitude with the leading order M(atrix)-Theory result we now turn to the question of whether the $\mathrm{M}$ (atrix)-Model is sensitive to the one-loop correction (5.21)to the M-Theory effective action. Performing a Fourier transform on (5.21) Гwe see that it scales like

$$
\frac{\ell_{p}^{24} v^{8}}{R_{11}^{7} r^{18}}
$$

Comparing with (5.1) $\Gamma$ we immediately see that this one-loop correction has a chance to match with the three-graviton M(atrix)-Model calculation pushed to one order higher in velocities than we have done above.

The setup of the computation is now clear: We simply expand all the terms in the two-loop effective action $\Gamma_{2 l o o p}$ of (5.7) to order $v^{8}$ and apply the same manipulations discussed above in this section to obtain the M(atrix)-Theory amplitudes.

The order $v^{8}$ result of the spin independent $1+2+3 \rightarrow 1^{\prime}+2^{\prime}+3^{\prime}$ amplitude is again comprised of three terms

$$
\left.S^{3 \rightarrow 3}\right|_{v^{8}}=\left.S_{o \multimap}^{3 \rightarrow 3}\right|_{v^{8}}+\left.S_{V}^{3 \rightarrow 3}\right|_{v^{8}}+\left.S_{Y}^{3 \rightarrow 3}\right|_{v^{8}}
$$

Dropping the overall energy and momentum conserving delta functions $\Gamma$ we find

$$
\begin{aligned}
\left.S_{\circ \rightarrow}^{3 \rightarrow 3}\right|_{v^{8}}=-\frac{\pi^{9}}{6}[ & \frac{v_{12}^{4} v_{31}^{4}}{\mathbf{q}_{2} \cdot \mathbf{v}_{12}}\left(\left\langle\frac{1}{\sigma_{1}^{2}}\right\rangle \mathbf{q}_{3} \cdot \mathbf{v}_{12}+\left\langle\frac{1}{\sigma_{2}^{2}}\right\rangle \mathbf{q}_{2} \cdot \mathbf{v}_{31}\right) \\
& +\mathbf{q}_{2} \cdot \mathbf{q}_{3} v_{12}^{4} v_{31}^{4}\left(\left\langle\frac{1}{\sigma_{1}}\right\rangle+\left\langle\frac{1}{\sigma_{2}}\right\rangle\right)
\end{aligned}
$$




$$
\begin{aligned}
& -4 \mathbf{v}_{12} \cdot \mathbf{v}_{31} v_{12}^{2} v_{31}^{2}\left(\left\langle\frac{1}{\sigma_{1}^{2}}\right\rangle v_{12}^{2}+\left\langle\frac{1}{\sigma_{2}^{2}}\right\rangle v_{31}^{2}\right) \\
& -4 \mathbf{q}_{2} \cdot \mathbf{v}_{12} v_{12}^{2} v_{31}^{2}\left(v_{12}^{2} \mathbf{q}_{2} \cdot \mathbf{v}_{31}+v_{31}^{2} \mathbf{q}_{3} \cdot \mathbf{v}_{12}\right)\left(\left\langle\frac{1}{\sigma_{1}}\right\rangle+\left\langle\frac{1}{\sigma_{2}}\right\rangle\right) \\
& \left.+16 \mathbf{v}_{12} \cdot \mathbf{v}_{31}\left(\mathbf{q}_{2} \cdot \mathbf{v}_{12}\right)^{2} v_{12}^{2} v_{31}^{2}\left(\left\langle\frac{1}{\sigma_{1}}\right\rangle+\left\langle\frac{1}{\sigma_{2}}\right\rangle\right)+\text { cyclic }\right]
\end{aligned}
$$

along with

$$
\begin{aligned}
\left.S_{V}^{3 \rightarrow 3}\right|_{v^{8}}= & \frac{\pi^{9}}{2} v_{31}^{4} v_{12}^{4}\left\langle\frac{1}{\sigma_{1} \sigma_{2}}\right\rangle-\frac{\pi^{9}}{3} \mathbf{v}_{12} \cdot \mathbf{v}_{31} v_{12}^{2} v_{31}^{2}\left[v_{12}^{2}\left\langle\frac{1}{\sigma_{1}}\right\rangle+\left\langle\frac{1}{\sigma_{2}}\right\rangle\right. \\
& \left.-4\left(\mathbf{q}_{2} \cdot \mathbf{v}_{12}\right)^{2}\left(\left\langle\frac{1}{\sigma_{1}}\right\rangle+\left\langle\frac{1}{\sigma_{2}}\right\rangle\right)\right]+ \text { cyclic }
\end{aligned}
$$

where we have used the abbreviation

$$
\left\langle f\left(\sigma_{1}, \sigma_{2}\right)\right\rangle=\int_{0}^{\infty} d^{2} \sigma f\left(\sigma_{1}, \sigma_{2}\right) e^{-\sigma_{1} q_{2}^{2}-\sigma_{2} q_{3}^{2}}
$$

i.e. the proper time integrals remain to be performed. As a matter of fact $\Gamma$ all integrals in (5.22) and (5.23) are divergent $\Gamma$ but exist in a distributional sense $\Gamma$ see for example [Z]. We first note that none of the terms in (5.22) and (5.23) displays the genuine two pole structure

$$
\langle 1\rangle=\frac{1}{q_{2}^{2} q_{3}^{2}}
$$

which found in the supergravity amplitude (5.21); such terms 5 however will arise from the $\left.S_{V}^{3 \rightarrow 3}\right|_{v^{8}}$ amplitude to be studied.

An immediate disagreement arises from the first term of (5.22) with a "re-scattering pole" $1 /\left(\mathbf{q}_{2} \cdot \mathbf{v}_{12}\right) \Gamma$ whereas on the supergravity side re-scattering diagrams of the type of Fig. 16 are absent since there are no $\mathcal{R}^{2}$ or $\mathcal{R}^{3}$ curvature corrections to the effective M-Theory action that could lead to three point vertices $\Gamma$ as argued above. Note also that $\left.S_{Y}^{3 \rightarrow 3}\right|_{v^{8}}$ does not give rise to re-scattering poles $\Gamma$ as we shall see shortly. Performing the corresponding $\sigma$ integrals for this term in a distributional sense

$$
\int_{0}^{\infty} d \sigma \frac{1}{\sigma^{2}} e^{-\sigma q^{2}}=\frac{1}{16} q^{2}\left(\log q^{2}+\gamma-1\right)
$$

where $\gamma$ is the Euler constant and the logarithm is to be understood as $\log q^{2} / \Lambda^{2}$ for some cut-off $\Lambda$ The re-scattering contributions of $\left.S^{3 \rightarrow 3}\right|_{v^{8}}$ take the form

$$
\frac{v_{12}^{4} v_{31}^{4}}{\mathbf{q}_{2} \cdot \mathbf{v}_{12}}\left(\frac{q_{2}^{2}}{q_{3}^{2}} \mathbf{q}_{3} \cdot \mathbf{v}_{12}+\frac{q_{3}^{2}}{q_{2}^{2}} \mathbf{q}_{2} \cdot \mathbf{v}_{31}\right)+\log \text { terms }
$$


Hence it is clear that M(atrix)-Theory produces terms with no counterpart on the supergravity side. HoweverTtaking a conservative point of view one could argue that only "truly eikonal" terms with a double pole $1 /\left(q_{2}^{2} q_{3}^{2}\right)$ structure should be compared on both sides. A similar phenomenon occurred in the polarization dependent two graviton scattering amplitudes [PSW] $\Gamma$ where the spin dependent contributions to the M(atrix)-Theory amplitude gave rise to terms canceling the $1 / q^{2}$ pole and had to be dropped because they are not reliable in the eikonal setup of classically free particles.

Taking this viewpoint we would have to conclude that all terms in (5.22) and (5.23) are spurious and we need to go on to the rather involved computation of $\Gamma_{Y}$ at order $v^{8}$. The outcome of this computation is the amplitude (remember that $p=\sigma_{1} \sigma_{2}+\sigma_{2} \sigma_{3}+\sigma_{3} \sigma_{1}$ )

$$
\left.S_{Y}^{3 \rightarrow 3}\right|_{v^{8}}=\int_{0}^{\infty} d^{3} \sigma \frac{1}{p^{5}} e^{-q_{1}^{2} \sigma_{2}-q_{2}^{2} \sigma_{3}-q_{3}^{2} \sigma_{1}}\left(\Upsilon^{2} p^{2} \Pi_{2}+\Upsilon p \Pi_{1}+\Pi_{0}\right)
$$

where $\Upsilon$ was introduced in (5.5) and where the $\Pi_{n}$ are polynomials of order $7-n$ in the $\sigma$ 's and of order $n$ in in $\mathbf{q} \cdot \mathbf{v}$ 's. In particular

$$
\Pi_{2}=-\frac{8 \pi^{9}}{3}\left(\left(\mathbf{v}_{12} \cdot \mathbf{q}_{1}\right)^{2}\left(\sigma_{1}+\sigma_{2}\right)\left(\sigma_{1} \sigma_{2}\right)^{2}-2 \mathbf{v}_{12} \cdot \mathbf{q}_{1} \mathbf{v}_{23} \cdot \mathbf{q}_{2} \sigma_{1} \sigma_{2}^{3} \sigma_{3}\right)+\text { cyclic }
$$

and

$$
\begin{aligned}
\Pi_{1}= & \frac{16 \pi^{9}}{3} \mathbf{v}_{12} \cdot \mathbf{q}_{1} \\
& \times\left(\left(\sigma_{1}^{2} v_{12}^{4}+\sigma_{2}^{2} v_{23}^{4}\right) \sigma_{1} \sigma_{2}\left(\sigma_{1} \sigma_{2}-2\left(\sigma_{1}+\sigma_{2}\right) \sigma_{3}\right)+3 v_{31}^{4}\left(\sigma_{1} \sigma_{2} \sigma_{3}\right)^{2}\right. \\
& +2 v_{12}^{2} v_{23}^{2} \sigma_{1} \sigma_{2}\left(\sigma_{1}^{3} \sigma_{2}+\sigma_{2}^{3} \sigma_{3}+3 \sigma_{1}^{2} \sigma_{2}^{2}+\sigma_{1}^{2} \sigma_{2} \sigma_{3}+\sigma_{1}^{3} \sigma_{3}+\sigma_{1} \sigma_{2}^{2} \sigma_{3}+\sigma_{1} \sigma_{2}^{3}\right) \\
& -v_{23}^{2} v_{31}^{2} \sigma_{2}^{2}\left(3 \sigma_{1}^{2} \sigma_{3}^{2}-\sigma_{2}^{2} \sigma_{3}^{2}-2 \sigma_{1}^{2} \sigma_{2} \sigma_{3}+\sigma_{1}^{2} \sigma_{2}^{2}+2 \sigma_{1} \sigma_{2} \sigma_{3}^{2}\right) \\
& \left.-v_{12}^{2} v_{31}^{2} \sigma_{1}^{2}\left(2 \sigma_{1} \sigma_{2} \sigma_{3}^{2}+\sigma_{1}^{2} \sigma_{2}^{2}-\sigma_{1}^{2} \sigma_{3}^{2}-2 \sigma_{1} \sigma_{2}^{2} \sigma_{3}+3 \sigma_{2}^{2} \sigma_{3}^{2}\right)\right)+ \text { cyclic }
\end{aligned}
$$

along with

$$
\begin{aligned}
\Pi_{0}=-\frac{8 \pi^{9}}{3}[ & v_{12}^{8} \sigma_{1}^{3}\left(-2 \sigma_{2}^{2} \sigma_{3}^{2}-\sigma_{1} \sigma_{2}^{2} \sigma_{3}-\sigma_{1} \sigma_{2} \sigma_{3}^{2}-4 \sigma_{1}^{2} \sigma_{2} \sigma_{3}+\sigma_{1}^{2} \sigma_{3}^{2}+\sigma_{1}^{2} \sigma_{2}^{2}\right) \\
& -4 v_{12}^{6} v_{23}^{2} \sigma_{1}^{2}\left(3 \sigma_{2}^{3} \sigma_{3}^{2}+\sigma_{1} \sigma_{2}^{2} \sigma_{3}^{2}+5 \sigma_{1}^{2} \sigma_{2}^{2} \sigma_{3}-\sigma_{1}^{2} \sigma_{2} \sigma_{3}^{2}-3 \sigma_{1}^{2} \sigma_{2}^{3}-\sigma_{1}^{3} \sigma_{2} \sigma_{3}\right. \\
& \left.+\sigma_{1}^{3} \sigma_{3}^{2}-2 \sigma_{1}^{3} \sigma_{2}^{2}\right)-2 v_{12}^{4} v_{23}^{2} v_{31}^{2} \sigma_{1}\left(\sigma_{1} \sigma_{3}-2 \sigma_{2} \sigma_{3}+\sigma_{1} \sigma_{2}\right)\left(\sigma_{1}^{2} \sigma_{3}^{2}-3 \sigma_{2}^{2} \sigma_{3}^{2}\right. \\
& \left.+\sigma_{1}^{3} \sigma_{3}-5 \sigma_{1}^{2} \sigma_{2} \sigma_{3}+\sigma_{1}^{3} \sigma_{2}+3 \sigma_{1}^{2} \sigma_{2}^{2}\right)+v_{12}^{4} v_{23}^{4}\left(2 \sigma_{1} \sigma_{2}^{5} \sigma_{3}-\sigma_{1} \sigma_{2}^{4} \sigma_{3}^{2}\right. \\
& +11 \sigma_{1}^{2} \sigma_{2}^{4} \sigma_{3}+10 \sigma_{1}^{2} \sigma_{2}^{3} \sigma_{3}^{2}+\sigma_{1}^{2} \sigma_{2}^{5}+10 \sigma_{1}^{3} \sigma_{2}^{2} \sigma_{3}^{2}+12 \sigma_{1}^{3} \sigma_{2}^{4} \\
& \left.\left.+11 \sigma_{1}^{3} \sigma_{2}^{2} \sigma_{3}-\sigma_{1}^{4} \sigma_{2} \sigma_{3}^{2}+12 \sigma_{1}^{4} \sigma_{2}^{3}+2 \sigma_{1}^{5} \sigma_{2} \sigma_{3}+\sigma_{1}^{5} \sigma_{3}^{2}+\sigma_{1}^{5} \sigma_{2}^{2}+\sigma_{2}^{5} \sigma_{3}^{2}\right)\right] \\
& + \text { permutations. }
\end{aligned}
$$


Note that the permutations in the above formula act on the "index objects" $\left(\mathbf{v}_{1}, \mathbf{q}_{1}, \sigma_{2}\right) \Gamma$ $\left(\mathbf{v}_{2}, \mathbf{q}_{2}, \sigma_{3}\right) \Gamma$ and $\left(\mathbf{v}_{3}, \mathbf{q}_{3}, \sigma_{1}\right)$ because of the coupling of proper times $\sigma_{i}$ and momenta $\mathbf{q}_{i}$ in the exponent of (5.24).

Amongst these terms it is now instructive to focus on a specific class of terms that have easily identifiable counterparts in the supergravity amplitude (5.21). We choose to study terms with the structure

$$
(\mathbf{q} \cdot \mathbf{v})^{4} \frac{v^{4}}{q^{4}}
$$

On the M(atrix)-Theory side these terms are easily isolated from $\left.S_{Y}^{3 \rightarrow 3}\right|_{v^{8}}$ of $(5.24) \Gamma$ in particular

$$
\left.S_{Y}^{3 \rightarrow 3}\right|_{v^{4}(\mathbf{q} \cdot \mathbf{v})^{4}}=\Upsilon^{2} \int_{0}^{\infty} d^{3} \sigma \frac{\Pi_{2}}{p^{3}} e^{-q_{1}^{2} \sigma_{2}-q_{2}^{2} \sigma_{3}-q_{3}^{2} \sigma_{1}}
$$

Of course $\Gamma$ it is rather hard to evaluate this integral exactly. Being interested only in the poles $1 /\left(q_{1}^{2} q_{2}^{2}\right)$ and permutations thereof we proceed as follows: First perform the integral over (say) $\sigma_{3}$ exactly and thereafter expand the integrand in powers of $1 / \sigma_{1}$ and $1 / \sigma_{2}$. Using

$$
\int_{0}^{\infty} d \sigma \frac{1}{\sigma} e^{-\sigma q^{2}}=-\log q^{2}-\gamma
$$

we obtain the final result contributing to the structure (5.25) (up to overall factors $\Gamma$ dropping the logarithms)

$$
\left.S_{2-\text { loop }}^{3 \rightarrow 3}\right|_{v^{4}(\mathbf{q} \cdot \mathbf{v})^{4}}=\Upsilon^{2}\left(\mathbf{q}_{1} \cdot \mathbf{v}_{12}\right)^{2}\left(\frac{1}{q_{2}^{2} q_{3}^{2}}+\frac{1}{q_{1}^{2} q_{2}^{2}}\right)+\text { cyclic }
$$

which is astonishingly close but nevertheless not equal to the corresponding terms in the supergravity amplitude of (5.21)

$$
\left.A_{\mathcal{R}^{4}}\right|_{v^{4}(\mathbf{q} \cdot \mathbf{v})^{4}}=\Upsilon^{2}\left(\mathbf{q}_{1} \cdot \mathbf{v}_{12}\right)^{2} \frac{1}{q_{3}^{2} q_{1}^{2}}+\text { cyclic. }
$$

This constitutes the above-mentioned definite disagreement of the two results and concludes our study of the $\mathcal{R}^{4}$ contributions to the three-graviton amplitudes.

Let us mention a further result found in [NP2]: There all possible contractions of four powers of the Riemann tensor were considered as possible terms in the supergravity effective action at one loop order. It was found that some of them do (up to a numerical factor) reproduce the same amplitude as the "correct" contraction whereas the remaining ones do not contribute at all in the kinematical setup we have chosen above. This means that the M(atrix)-Theory amplitude we have derived cannot be obtained from a Lorentz invariant theory in eleven dimensions. It might be necessary to add further terms to the $\mathrm{M}$ (atrix)-Theory action to render it covariant. Such corrections are not uncommon for theories in light-cone gauge. 


\subsection{Why the Discrepancy?}

In this chapter $\Gamma$ we have presented detailed comparisons between three-graviton scattering amplitudes in $\mathrm{M}$ (atrix)-Theory and $d=11$ supergravity along with its leading M-theoretic higher derivatives corrections. On the one hand $\Gamma$ we have been able to complete and unify the results of [OY1] and [OY2] showing that the leading order $v^{6}$ eikonal spin independent $S$-matrices of tree level supergravity and one-loop M(atrix)-Theory exactly agree. On the other hand $\Gamma$ the moment one studies the next-to-leading $v^{8} \mathrm{M}$ (atrix)-Theory amplitude $\Gamma$ the result fails to match the corresponding (conjectured) term in $\mathcal{R}^{4}$ corrected supergravity. Why does such a mismatch occur?

There are several different possible levels of explanation. The first is to ask why one might have expected agreement in the first place. Both the M(atrix)-Model-conjecture that relates a gauge theory to M-Theory (and especially $\Gamma$ as we have studied it in this thesis to its gravity limit) and the AdS-CFT correspondence that relates a conformally invariant gauge theory on the boundary of anti de Sitter space to gravity in its bulk $\Gamma$ use string theory as an intermediate step to motivate or explain the correspondence. In both cases $\Gamma$ there is then a duality between a gauge theory and a gravitational interpretation of the stringy setup.

But a closer investigation reveals that the two descriptions apply in different corners of the moduli space of M-Theory: From a stringy perspective $\Gamma$ we see that the description in terms of stretched open strings stretching is appropriate if the particles are close and the open strings are short and therefore light. On the other hand $\Gamma$ we would trust a description in terms of classical gravity if we can neglect higher curvature corrections. This approximation is justified if curvature is small everywhere. But this is equivalent to saying the probe particles should be far away from the source for the supergravity description to be valid. Only in special cases $\Gamma$ the scaling behavior of the effective force between the particles will show the same scaling behavior in both regimes as we encountered it in the case of the $v^{4}$ and $v^{6}$ terms. It is highly plausible that supersymmetry is responsible for this protection of amplitudes. The $\mathcal{R}^{4}$ term we have been investigating is the first amplitude calculated in both descriptions that is not believed to be protected by supersymmetry and in fact cannot be protected as we found a disagreement between the two different regimes.

This discussion of regimes can also be made more quantitative: The description in terms of open strings is justified if the length of the strings is much shorter than the string scale $\ell_{s}=\sqrt{\alpha^{\prime}}$. In that case $\Gamma$ the strings are light while the higher modes are heavy and Yang-Mills theory is a good approximation to the Born-Infeld action that includes higher order terms in $\alpha^{\prime}$. On the other hand $\Gamma$ we expect supergravity to be valid at distances that are large compared to the Planck scale $\ell_{p}$. More concretely $\Gamma$ the supergravity solution that describes a D0-brane at macroscopic distances has a curvature[IMSY]

$$
\alpha^{\prime} R=\sqrt{\frac{\left(r / \alpha^{\prime}\right)^{3}}{g_{\mathrm{YM}}^{2} N}}
$$


at distance $r$ from the center. Here $\Gamma$ we reinserted the M(atrix)-Theory coupling $g_{\mathrm{YM}}$. As the coupling appears in the denominator $\Gamma$ it is obvious that the requirements of small curvature and small coupling (such that perturbation theory is valid) are in conflict. We cannot expect classical gravity and Yang-Mills theory to hold at the same time $N$ finite.

Another possible explanation for the disagreement is that supergravity in eleven uncompactified dimensions is not the right theory to compare to. The light like limit we have to take for finite $N$ bears a subtlety in its definition.

As we explained in the previous section $\Gamma$ the two amplitudes on the supergravity and on the $\mathrm{M}$ (atrix)-Theory side $\Gamma$ respectively $\Gamma$ scale differently with respect to $N \Gamma$ the momentum in the compactified direction. In the original M(atrix)-Model conjecture put forward in [BFSS] $\Gamma N$ has to be taken to infinity to recover uncompactified M-Theory. As the two amplitudes of supergravity and the M(atrix)-Model respectively scale differently with $N \Gamma$ they are no longer comparable. Rather one should consider the non-orientable five-loop diagram on the M(atrix)-Model side that one obtains from world sheet-duality $\Gamma$ as we explained earlier.

On the other hand $\Gamma$ if one seriously tries to approach the problem of the large $N$ limit (for some progress in this directionГsee e.g. [KNS]) $\Gamma$ it is no longer sufficient to take the diagonal matrices we have been using as the background. Rather one would have to use the true ground state wave function of the $N_{a}$ model to describe the configuration of parton $a$ with compact momentum $N_{a}$. As long as no further information in this direction is available (but $\Gamma$ see [FGHHY]) $\Gamma$ one has to restrict oneself to finite $N$ calculations in which each parton carries momentum $N_{a}=1$. In this setup $\Gamma$ the scaling with $N$ is invisible and the matrix diagram we have been considering stands on the same level as the five-loop non-orientable diagram in Fig. 22.

For finite $N$ Tthe original proposal of [BFSS] does not applyCrather we have to use the second proposal of [Su]. There Tone has to consider M-Theory compactified on a light-like circle. This so-called Discrete Light-Cone Quantization (DLCQ) was used at some time in canonical approaches to quantum chromo-dynamics because it promised to significantly simplify the structure of the QCD vacuum.

As it turned out once again $\Gamma$ there is no such thing as free lunch and DLCQ is plagued by conceptual problems that we will describe here because there are indications they are connected to the problem of the mismatch between the supergravity and M(atrix)Theory amplitudes. These well-known problems were first investigated in the context of the M(atrix)-Model in [HP].

As a simple example we consider the DLCQ of a scalar field. To do soГwe use a metric

$$
d s^{2}=-2 d \tau d x^{-}+\epsilon^{2} d x^{-} d x^{-}+d x^{i} d x^{i}
$$


with a small parameter $\epsilon$. In this metric $\Gamma$ the norm of the vector field $\partial_{-}$is

$$
\left\|\frac{\partial}{\partial x^{-}}\right\|=\epsilon
$$

Therefore $\Gamma x^{-}$becomes light-like once we take the limit $\epsilon \rightarrow 0$. We compactify this direction by imposing

$$
x^{-} \cong x^{-}+2 \pi R_{11}
$$

Then Twe Fourier transform the scalar field in the compact direction:

$$
\phi\left(\tau, x^{-}, x^{i}\right)=\frac{1}{\sqrt{2 \pi R_{11}}} \sum_{n} \phi_{n}\left(\tau, x^{i}\right) e^{i n \frac{x^{-}}{R_{11}}}
$$

Writing out the mode expansion $\Gamma$ the action becomes

$$
\begin{aligned}
\mathcal{S}= & \int d^{d} x\left(\partial_{\mu} \phi^{*} \partial^{\mu} \phi+m^{2} \phi^{*} \phi+\text { interactions }\right) \\
= & \sum_{n} \int d \tau \int d^{d-2} x_{\perp}\left(\epsilon^{2} \partial_{\tau} \phi_{n}^{*} \partial_{\tau} \phi_{n}+\frac{2 i n}{R_{11}} \phi_{n}^{*} \partial_{\tau} \phi_{n}-\partial_{i} \phi_{n}^{*} \partial_{i} \phi_{n}\right. \\
& \left.\quad+m^{2} \phi_{n}^{*} \phi_{n}+\text { interactions }\right) .
\end{aligned}
$$

Now $\Gamma$ we would like to take the light-like limit $\epsilon \rightarrow 0$. This removes the first term with two $\partial_{\tau}$ derivatives. For the zero mode $\phi_{0} \Gamma$ also the second term vanishes. Thus $\Gamma$ in a canonical treatment $\Gamma$ we see that its conjugate momentum

$$
\pi_{0}=\frac{\delta \mathcal{S}}{\delta \partial_{\tau} \phi_{0}}=0
$$

is identically zero. This means that $\phi_{0}$ is an auxiliary field and not dynamical. It gives rise to a constraint

$$
\dot{\pi}_{0}=\frac{\delta \mathcal{S}}{\delta \phi_{0}} \stackrel{!}{=} 0
$$

This has to be solved for $\phi_{0}$ and the solution has to be re-inserted into the action. In general $\Gamma$ this yields an infinite series of interactions that make the theory very difficult to deal with.

In the path integral approach that we have been using in this thesis $\Gamma$ the problem appears as follows: To calculate Feynman graphs in momentum representation Tone would use the propagator

$$
G_{n}=\frac{i}{\epsilon^{2} p_{\tau}^{2}+\frac{2 n}{R_{11}} p_{\tau}-p_{i} p_{i}-m^{2}}=\frac{i}{p_{\omega}^{2}-\frac{n^{2}}{\epsilon^{2} R_{11}^{2}}-p_{i} p_{i}-m^{2}}
$$


for $\phi_{n} \Gamma$ where we have performed the transformation

$$
p_{\omega}=\epsilon p_{\tau}+\frac{n}{\epsilon R_{11}} \text {. }
$$

In the second form $\Gamma$ the propagator $G_{n}$ is just the ordinary propagator for a scalar field with a modified mass in $d-1$ dimensions. But $\Gamma$ what is more $\Gamma$ in an $L$ loop diagram $\Gamma$ there are $L$ loop momenta that give contribute

$$
\int\left(d^{d} p\right)^{L}=\frac{1}{\epsilon^{L}}\left(\int d p_{\omega} d^{d-1} p_{i}\right)^{L}
$$

after the above transformation. We find that in an $L$ loop computation the light-like limit leads to an overall $\epsilon^{-L}$ divergence in all amplitudes.

In the above argument we have not used any specific properties of scalar field theory. Rather $\Gamma$ the divergence encountered seems to be a generic feature of all field theories. This applies also to supergravity if it is viewed as a quantum field theory as we have been doing it above. The fact that the divergences scale like $\epsilon^{-L}$ agrees well with the observation that M(atrix)-Theory has been successfully compared to $L=0$ tree-level gravity whereas the comparison to $L=1$ supergravity fails.

This would suggest that the light-like limit of field theories is not well defined at least in the naïve sense. What we have compared our M(atrix)-Theory result to is elevendimensional supergravity trivially reduced along the light-like circle. On the other hand $\Gamma$ we just have seen this is not allowed as it leads to divergences.

Here $\Gamma$ a historical remark is in order: At the time when [HP] came out $\Gamma$ there was a need to explain the disagreement found in $[\mathbf{D R}] \Gamma$ and the authors of the former paper suggested that the subtleties of the DLCQ limit might be responsible for the disagreement. The authors did not recognize that one should expect the problems of DLCQ only for loop amplitudes of the DLCQ field theory. In the disagreement reported on in [DR] ${ }_{\text {supergravity }}$ as the DLCQ theory was treated in the tree level approximation. SoГstrictly speaking $\Gamma$ the argument in [HP] cannot explain the disagreement in [DR] that we now know to be based on a calculational error as impressively demonstrated in [OY1]. In contrast $\Gamma$ the disagreement we found in our above calculation is based on a loop calculation in supergravity in the DLCQ limit and the problem reported on in [HP] might well affect it.

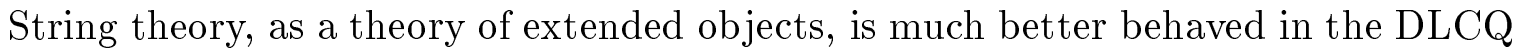
limit. Bilal $[\mathbf{B i}]$ has calculated the string one-loop partition function in DLCQ and found it to be finite. Therefore $\Gamma$ it is reasonable to expect that also $\mathrm{M}$-Theory $\Gamma$ as a theory of extended objects is finite in the DLCQ limit. What is more Cour results suggest that it is not a simple reduction of eleven-dimensional supergravity that describes M-Theory in this limit. In view of the "derivations" of the M(atrix)-Model by Seiberg[Sei] and Sen[Se] [we are led to believe that the DLCQ limit is just another corner of the M-Theory moduli space that happens to be well described by the M(atrix)-Model. There is some overlapГpossibly 
mediated by supersymmetry $\Gamma$ with the-low energy corner of uncompactified supergravity $\Gamma$ but for more sophisticated processes $\Gamma$ the corners are distinct.

Our first argument about the different regimes implied by the supergravity and gauge theory approximation used stringy scaling arguments to explain why at least it is not possible to use perturbative string theory to prove that all supergravity amplitudes have to be reproduced by the M(atrix)-Model. The argument we gave above suggests that any amplitude that is not protected by supersymmetry will in general not be the same in the sub-stringy and in the macroscopic regimes where we expect the two descriptions to be valid. But we did not show that we should have expected a disagreement. The other argument suggested that in the DLCQ limit of a field theory there are divergences that at least make it difficult to relate DLCQ loop amplitudes to amplitudes in the uncompactified theory. So it might still be that our M(atrix)-Theory result matches DLCQ supergravity (or even DLCQ M-Theory) once there is a sensible definition of this limit and a procedure to deal with the $\epsilon^{-L}$ divergencies $\Gamma$ possibly again coming from string theory as suggested in $[\mathbf{B i}]$ is adopted.

There is a calculation that is just beyond our present technical ability that would be able to differentiate between lacking protection by supersymmetry and problems arising from loops in a DLCQ field theory. This is the test whether M(atrix)-Theory is able to reproduce the third order $v^{8}$ term in (4.6) or the $c_{33}$ term in (5.1). It is not believed that this term is protected by a supersymmetric non-renormalization theorem $\Gamma$ at least the techniques used to prove[PSS1][PSS2][NP1] the supersymmetric protection of the terms at order $v^{4}$ and $v^{6}$ do not apply and do not easily generalize to $v^{8}$. Furthermore $\Gamma$ [DEG] argue that IR problems are to be expected at this order. On the other hand $\Gamma$ the corresponding calculation on the supergravity side uses only tree-level so that we do not expect the DLCQ limit to complicate the correspondence as we explained above. While the expected value from supergravity can be calculated almost trivially by expanding (4.6) one order further $\Gamma$ the difficulty of obtaining this amplitude on the M(atrix)-Model side lies solely in the complexity of three-loop gauge theory calculations $\Gamma$ with about one hundred diagrams and up to six proper time integrations.

An even more involved investigation would be the calculation of the M(atrix)-Model process that is directly related to the one loop gravity amplitude by world sheet-duality.

It is not obvious to find the corresponding open string diagram. For the identification convenient to note topological invariants: Corresponding to the four external states $\Gamma$ there are four boundaries and the Euler number turns out to be

$$
\chi=2 g-2+b=4 .
$$

As noted already in [KVK1][Ser] $\Gamma$ the non-planarity of the diagram corresponds to sub-leading $\frac{1}{N}$ behavior on the M(atrix)-Theory side. We have to look for a non-planar ribbon diagram with four boundaries and we find it to be

The calculation of the Euler number is most easily performed by cutting the diagram into 


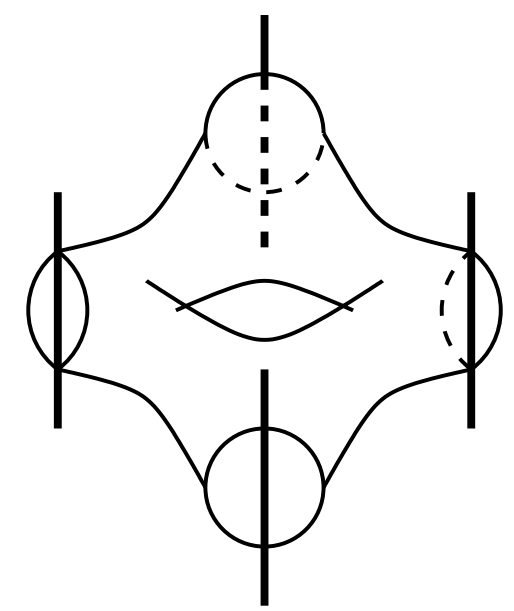

Fig. 23: The $\mathcal{R}^{4}$ string diagram

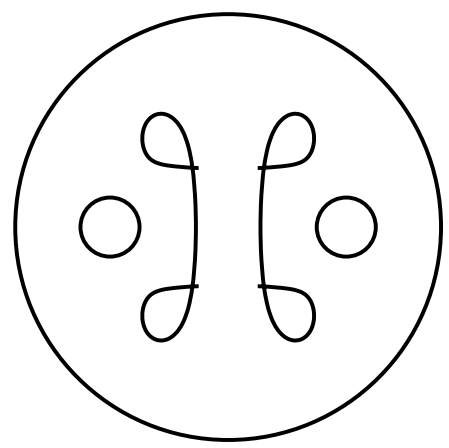

Fig. 24: The corresponding gauge theory ribbon diagram

simply connected polygons and using the discrete version of the Euler number calculation

$$
-\chi=\# \text { vertices }-\# \text { edges }+\# \text { surfaces }=-4 \text {. }
$$

This surface obviously also has four boundaries. Thinning out the ribbon to lines and moving the handle to the right $\Gamma$

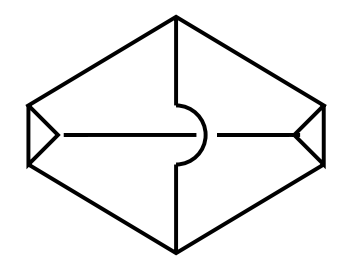

Fig. 25: The ribbon diagram as ordinary Feynman diagram

we see that this diagram arises at five-loop order. This calculation is definitely beyond our technical abilities. But as we have pointed out above $\mathrm{t}$ to be able to perform large $N$ calculations one has to have access to the internal structure of the D0-branes given by the ground state wave function whereas at finite $N$ the contribution of this diagram is 
indistinguishable from the two loop contribution we have calculated above. Furthermore $\Gamma$ we see from the table given in (5.1) that at five loop level there is no term with the correct scaling with $r$ to match the gravity amplitude we have derived above. 


\section{Beyond the Eikonal Regime}

All scattering calculations that have been performed so far in the M(atrix)-Model proceed in the same way namely by calculating quantum fluctuations around the diagonal classical solution. The particles described by this solution do not interact at all at the classical level and all interactions merely occur as quantum effects. Therefore $\Gamma$ the interactions appear only as perturbations so that the particles only depart just infinitesimally from their straight line motion. The effect we have been calculating so far as the effective action $\Gamma$ is $\Gamma$ after integration over the particles' proper timeГjust a phase shift. As we have stressed already this corresponds to an eikonal limit of scattering processes.

Technically $\Gamma$ this means that $S$-matrix elements can only be evaluated at the maximal $t$-pole $\Gamma$ i.e. in the limit of vanishing momentum transfer. It would be highly desirable to drop this restriction $\Gamma$ so one would be able to explore a much wider kinematical regime. Furthermore this restriction will have to be overcome if the full eleven-dimensional Lorentz invariance of the M(atrix)-Model is to be shown some day.

The issue of momentum transfer comes in two similar though distinct versions: The momentum transferred can either be in the transversal directions or in the compactified light-like direction. The first amounts to particles with different asymptotic velocities for incoming and for outgoing particles. The latter possibility is described by block diagonal matrices in which the size of the blocks changes during the interaction.

Processes of the first kind are included in the supergravity scattering set-ups we have been studying and had to be excluded later by extracting only the most singular terms with respect to momentum transfer $\Gamma$ whereas processes with momentum transfer of the second kind were excluded by the choice of kinematical setup.

The key question to be able to deal with processes with any of the two kinds of momentum transfer is whether one can find a classical solution that already accomplishes momentum transfer. On such a background it would be possible to study quantum fluctuations that could then be related to momentum-transferring interactions on the supergravity side. It is the aim of this chapter to study these non-eikonal classical solutions. In the end $\Gamma$ it will turn out that — at least for a toy model — no such solutions exist for finite $N$ and again one has to resort to the large $N$ limit. We will give good reasons why we think that also the full M(atrix)-Model has no scattering solutions with momentum transfer. Most of the material presented in this chapter is original and has not been published before.

\subsection{Momentum Transfer in Higher Dimensions and $N=\infty$}

Classical solutions to the M(atrix)-Model equations of motion that describe momentum transfer have been studied in $[\mathbf{P P}] \Gamma[\mathbf{K V K} \mathbf{2}]$ and $[\mathbf{G H V}]$. All the three works have one restriction in common that we would like to avoid here: They provide solutions only for infinite matrices $\Gamma$ i.e. for $S U(\infty)$. In particular $\Gamma$ the latter reference constructs a solitonic solution that 
describes light-like momentum transfer. Unfortunately tion for the M(atrix)-Model but for M(atrix)-String Theory[DVV] $\Gamma$ the $(1+1)$-dimensional super Yang-Mills theory that is obtained from the M(atrix)-Model by T-dualizing in one dimension. Matrix-String Theory is supposed to encode the dynamics of D-strings $\Gamma$ the images of fundamental strings under S-duality in type IIB string theory. As we have described in chapter $3 \Gamma$ this T-dualization requires infinite matrices to capture the effects of the infinite lattice of mirror D-particles. The solution is obtained by giving a complex structure to the $(1+1)$-dimensional "space-time" of the gauge theory and then translating the supersymmetric "square root" $Q \psi=0$ of the field equations into a holomorphicity condition. The latter can be solved by an analytic ansatz incorporating boundary conditions which describe the scattering kinematics. As should have become clear $\Gamma$ this approach depends crucially on the world-volume of the D-string being two-dimensional and cannot be translated to the M(atrix)-Model in a straightforward way.

The descriptions in $[\mathbf{P P}]$ and in [KVK2] also use T-duality to first transform the field theory to a higher-dimensional one. In that case $\Gamma$ two dimensions are T-dualized. The authors obtain a $U(2)$ gauge theory in $2+1$ dimensions which $\Gamma$ for separated $\mathrm{D} 2$-branes $\Gamma$ is spontaneously broken to $U(1) \times U(1)$. Here $\Gamma$ the transfer of compact momentum in the $\mathrm{M}$ (atrix)-Model appears as a solitonic process that transfers magnetic flux from one $U(1)$ factor to the other. Again $\Gamma$ we conclude using Taylors M(atrix)-Model version of T-duality $\Gamma$ that we end up with infinite matrices in the $(0+1)$-dimensional theory.

As we have mentioned already $\Gamma$ here $\Gamma$ we prefer to take a more conservative point of view and restrict ourselves to finite $N$ as long as further input from the ground state wave functions seems not to be available. If there is some truth to the finite $N \mathrm{M}$ (atrix)Model conjecture of $[\mathrm{Su}] \Gamma$ this model $\Gamma$ too $\Gamma$ should possess momentum transferring classical solutions.

As we have seen in chapter fourCleading order polarization independent processes are described by purely bosonic backgrounds. Fermionic backgrounds encode polarization dependent effects. Thus Tone expects a momentum transferring solution already in the bosonic model $\Gamma$ and for the question of existence of such solutions $\Gamma$ we can neglect the fermionic degrees of freedom.

This should not be confused with the findings of [dWLN]: There $\Gamma$ it was proven that $\Gamma$ in the supersymmetric model the Hamilton operator has a continuous spectrum while the Hamilton operator of the purely bosonic model has a discrete spectrum. The reason was that $\Gamma$ in the bosonic case $\Gamma$ the Heisenberg uncertainty principle prevents the wave function to leak out to infinity through the valleys of the potential that we investigate in this chapter. The zero point energy that we found in chapter three to be responsible for the confinement of the D0-particles in the bosonic model lifts $\Gamma$ in the language of [dWLN] $\Gamma$ the bottom of the potential valleys. This lifting does not occur in the supersymmetric model as supersymmetry requires the zero point energy to vanish. Therefore $\Gamma$ in the model with fermions $\Gamma$ the wave function can leak out; it is no longer confined and the spectrum is continuous. 
At this point it is important to note that this is a consequence of zero point energies and the uncertainty principle. Both are genuine quantum effects. The question about the existence of solutions to the equations of motion we investigate in this chapter is a purely classical one and thus we do not expect the picture to be qualitatively different between the bosonic and the supersymmetric version of the model.

Let us $\Gamma$ for concreteness $\Gamma$ fix $N=2$ and consider only bosonic degrees of freedom from now on. Then we can use a vector notation for $S U(2)$ indices $\Gamma$ and the Lagrangian reads

$$
\begin{aligned}
L & =\frac{1}{2} \sum_{i} \dot{\vec{X}}^{i} \cdot \dot{\vec{X}}^{i}-\sum_{i \neq j}\left\|\vec{X}^{i} \times \vec{X}^{j}\right\|^{2} \\
& =\frac{1}{2} \sum_{i} \dot{\vec{X}}^{i} \cdot \dot{\vec{X}}^{i}-\sum_{i \neq j}\left\|\vec{X}^{i}\right\|^{2}\left\|\vec{X}^{j}\right\|^{2}+\sum_{i \neq j}\left(\vec{X}^{i} \cdot \vec{X}^{j}\right)^{2} .
\end{aligned}
$$

Because we have gauged away the $A$ field $\Gamma$ we furthermore have to impose gauge invariance in the form of a Gauß law:

$$
\sum_{i} \vec{X}^{i} \times \dot{\vec{X}}^{i}=0
$$

The equation of motion is found to be

$$
\ddot{X}^{i}=-\vec{X}^{i} \sum_{j \neq i}\left\|\vec{X}^{j}\right\|^{2}+\sum_{j \neq i}\left(\vec{X}^{j} \cdot \vec{X}^{i}\right) \vec{X}^{j} .
$$

Furthermore $\Gamma$ for some time $t=t_{0} \Gamma$ we can use the $S O(9)$ invariance to adapt the coordinate system to the "main axis" as

$$
\vec{X}^{i}\left(t_{0}\right) \cdot \vec{X}^{j}\left(t_{0}\right)=0 \quad \text { for all } i \neq j
$$

so that the second term in the equation of motion vanishes for $t=t_{0}$.

As we are looking for backgrounds for the quantum scattering computations $\Gamma$ we are only interested in solutions that come in from infinity asymptotically infinite separation of the particles. We are not interested in bound solutions that stay in the vicinity of the origin forever. In the language of chapter three $\Gamma$ we only look for solutions where $\|\vec{r}\|$ goes to infinity for early and late times. There $\Gamma$ we fixed a gauge by requiring the matrices to be diagonal. Since we are now interested in more general solutions we pose this requirement in a gauge invariant way:

$$
R^{2}:=\sum_{i}\left\|\vec{X}^{i}\right\|^{2}, \quad \lim _{t \rightarrow \pm \infty} R^{2}=\infty
$$

At time $t_{0} \Gamma$ the potential energy is given by

$$
V=\sum_{i \neq j}\left\|\vec{X}^{i}\right\|^{2}\left\|\vec{X}^{j}\right\|^{2}
$$


For some $i \Gamma$ the squared length $\left\|\vec{X}^{i}\right\|^{2}$ has to be at least $R^{2} / 9$ which goes to infinity. As $V$ is bounded from above by the total energy directions $j$ of $\left\|\vec{X}^{j}\right\|^{2}$ have to be small and go to zero for late times. Of all contributions to $R^{2}$ Tone of the $\vec{X}^{i}$ 's largely dominates the others by at least two powers of $R$.

\subsection{The Toy-Model}

In this section Twe will study a simplified model that has only two degrees of freedom whose interactions mimic the ones found in M(atrix)-Theory. (Classical solutions to this model were also studied in [BMM] and [AMRV] but from the point of view of chaotic dynamics.) We will first give a handwaving argument why one does not expect this toy-model to have scattering solutions and then present a mathematical proof for the absence of scattering solutions. In the next section $\Gamma$ we will show that $\Gamma$ for asymptotic times $\Gamma$ the full $\mathrm{M}(\mathrm{a}-$ trix)-Model can be related to the truncated toy-model and therefore it has no scattering solutions either besides the diagonal ones we have presented in chapter 3 already.

We have seen in chapter 4 that the off-diagonal matrix elements are described by harmonic oscillators for large separations of the particles whose coordinates are given by the diagonal matrix elements. The frequency is proportional to the separation of the "diagonal" particles. The energy landscape has flat valleys in the directions of diagonal matrices that grow steeper and narrower the larger the separation of the particles gets.

There is a simple toy model with similar valleys in the potential energy landscape which was already used in [dWLN] to present the strategy for the proof of the continuity of the M(atrix)-Model spectrum. It should be thought of as a cartoon of the M(atrix)-Model after global rotation symmetries have been fixed. In this model $\Gamma$ there are two real degrees of freedom $\Gamma x(t)$ and $y(t) \Gamma$ with dynamics given by the Lagrangian

$$
L=\frac{1}{2}\left(\dot{x}^{2}+\dot{y}^{2}-x^{2} y^{2}\right) .
$$

The potential $V=x^{2} y^{2} / 2$ is non-negative and vanishes along the coordinate axes in the $(x, y)$ plane. Perpendicular to the axes $\Gamma$ the potential is quadratic $\Gamma$ the corresponding harmonic oscillator has a frequency given by the distance to the origin.

The equations of motion

$$
\begin{aligned}
& \ddot{x}=-x y^{2} \\
& \ddot{y}=-y x^{2}
\end{aligned}
$$

have trivial solutions if both sides vanish simultaneously

$$
x(t)=b+v t, \quad y(t)=0 ;
$$

these should be viewed as analogs of the diagonal solutions of the M(atrix)-Model. Here $\Gamma$ $b$ and $v$ are integration constants. Trivially $\Gamma$ there is another solution obtained by interchanging $x$ and $y$. Next $\Gamma$ we would like to find out if there can be further scattering solutions. 


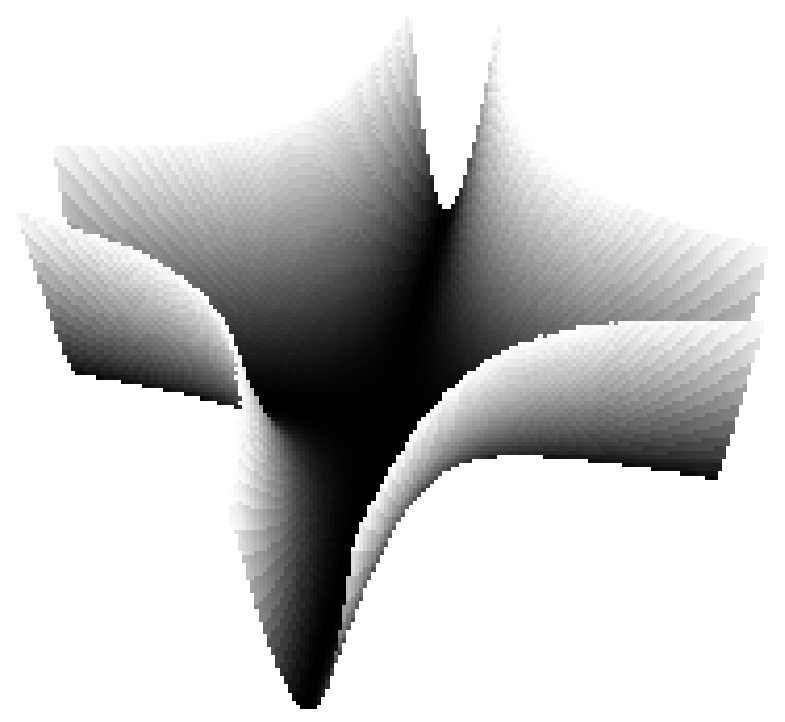

Fig. 26: The potential of the toy-model

By the name "scattering solution" we mean a solution for which

$$
R(t):=\sqrt{x^{2}+y^{2}}
$$

becomes infinite for $t \rightarrow \pm \infty$. This limit is meant in the usual sense that for each $R_{0}$ there is a time $T$ S such that $R(t)>R_{0}$ for all $|t|>T$. We are not interested in solutions that enter the valleys but always return to the "stadium" around the origin after every such excursion $\Gamma$ although $R(t)$ might not be bounded for such solutions.

We can use the $\pm x \leftrightarrow \pm y$ symmetry to assume without loss of generality that the solution escapes along the positive $x$-axis for late times. From the equation of motion see that also $\dot{x}$ has to be positive after $T$ because otherwise the velocity will stay negative until $x=0$ and we are back to the stadium again. As we have argued above $\Gamma$ we expect a motion that is mainly directed along the $x$-axis but with small oscillations in $y$ that are bound by the valleys. There are two possible scenarios: Either these oscillations will get smaller and smaller as the valleys are getting narrower and narrower or the oscillations are so strong that $\Gamma$ eventually $\Gamma$ the component of the gradient of the potential in negative $x$ direction off the bottom of the valley will stop the motion in the $x$-direction and force the particle to return to the stadium.

Numerical evolutions of the equations of motion indicate that the latter behavior is generic but we would like to investigate if there can be exceptions other than the trivial $y=0$ solution we have given above.

It is instructive to split the energy into two contributions coming from the motions in the $x$ and $y$ direction:

$$
E=E_{x}+E_{y}, \quad E_{x}=\frac{1}{2} \dot{x}^{2}, \quad E_{y}=\frac{1}{2}\left(\dot{y}^{2}+x^{2} y^{2}\right)
$$




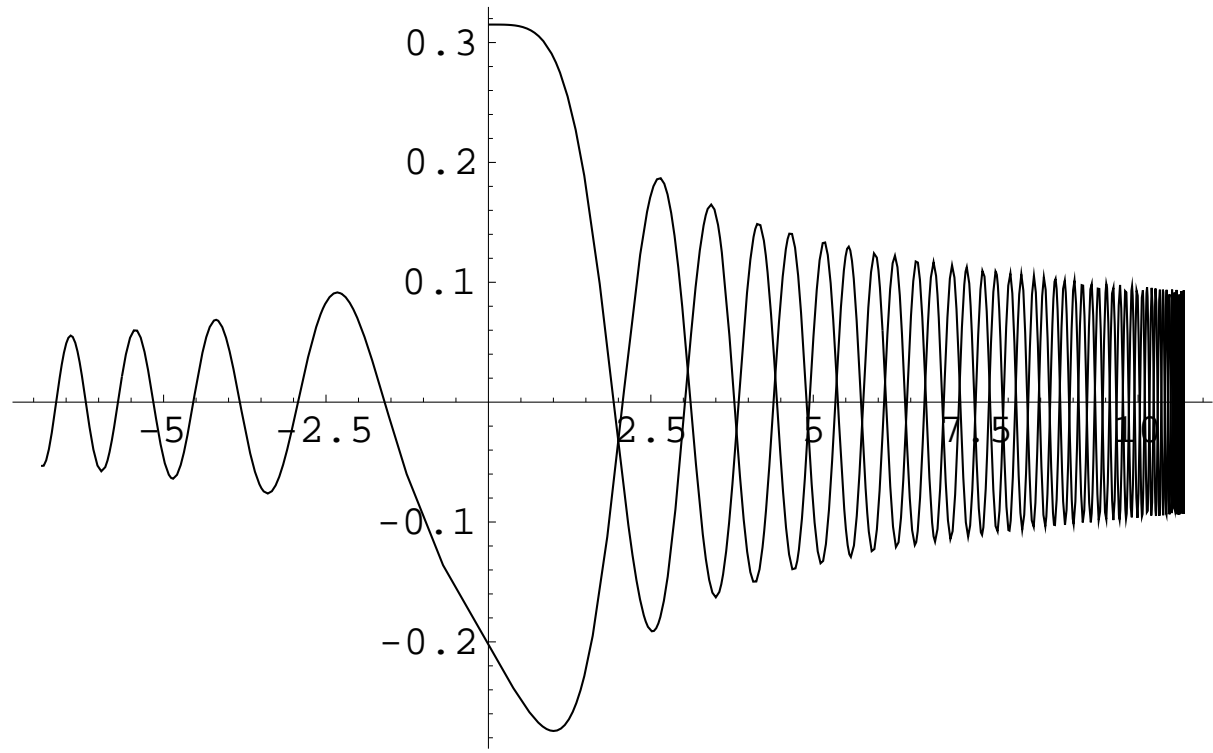

Fig. 27: A numerical example: The solution of the toy model returns to the stadium

Taking the time derivative $\Gamma$

$$
\dot{E}_{y}=-\dot{E}_{x}=-\ddot{x} \dot{x}=x \dot{x} y^{2} \geq 0
$$

where we used the assumptions $x>0$ and $\dot{x}>0$ from above. We see that energy is transferred constantly from the $x$ motion to the oscillations in the $y$ direction. Thus $\Gamma$ if we took the energy $E_{y}$ to be constant we would underestimate the oscillations.

Let us recall one fact about the harmonic oscillator: It follows from the virial theorem $\left\langle E_{\text {kin }}\right\rangle=\left\langle E_{\text {pot }}\right\rangle$ that the time average of the square of the oscillating variable $\left\langle\phi^{2}\right\rangle$ is given by

$$
\left\langle\phi^{2}\right\rangle=\frac{E}{\omega^{2}}
$$

in terms of the energy $E$ and the frequency $\omega$. Since $x \Gamma$ the frequency of the oscillations in $y \Gamma$ is assumed to go to infinity $\frac{1}{x} \Gamma$ the timescale of the oscillations $\Gamma$ is going to zero. Thus $\Gamma$ for late times $\Gamma$ the variation of the frequency during one period of the oscillation becomes smaller and smaller. Therefore $\Gamma$ in the equation of motion for $x \Gamma$ we can replace the effect of the oscillations by the average over one period and use (6.2):

$$
\ddot{x}=-x y^{2} \approx-x\left\langle y^{2}\right\rangle=-x \frac{E_{y}}{x^{2}}=-\frac{E_{y}}{x}
$$

This force on $x$ can be described by an effective potential as

$$
V_{\text {eff }}(x)=E_{y} \log x
$$

As the logarithm grows without bound $\Gamma$ the motion in $x$ will hit a potential barrier no matter how big the total energy $E$ is. The only exception would be that $E_{y}=0$ but 


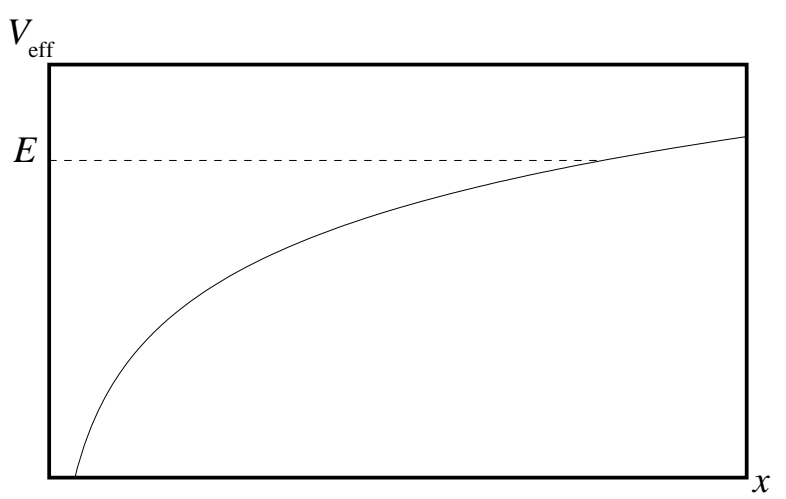

Fig. 28: The effective potential

this is again the trivial solution without oscillations. Therefore $\Gamma$ we do not expect to find any other solutions that escape to infinity.

One might worry that the above reasoning using the adiabatic time averaging might be not justified. Therefore $\Gamma$ we will give a more formal proof of the non-existence of scattering solutions $\Gamma$ next. To this end $\Gamma$ we will proceed along the lines of the previous heuristic argument $\Gamma$ but without using any a priori knowledge about the solution. Therefore $\Gamma$ we cannot employ the expressions for the harmonic oscillator in the $y$ direction.

Our strategy will be to assume that there is a solution $(x(t), y(t))$ with $E_{y}>0$ for which $x(t)$ goes to infinity as $t \rightarrow \infty \Gamma$ and to show that this assumption leads to a contradiction. Namely we will show that under these assumptions

$$
\int_{0}^{\infty} d t \ddot{x}=-\infty
$$

This implies that any velocity in the $x$ direction will be stopped and $x$ will eventually become negative again. Thus only the trivial solutions mentioned above will escape to infinity. Of course $\Gamma$ as the model is invariant under time reversal $\Gamma$ this also means that every solution that comes from $x=-\infty$ in the past has to be trivial.

To begin with $\Gamma$ let us recall that we can assume $x$ to be arbitrarily large and that $\dot{x}$ is positive. Furthermore $\Gamma E_{y}$ is strictly increasing in time and since the total energy is conserved $\Gamma$ all velocities and $y$ are bounded by constants determined by the initial conditions.

For some $t_{0}$ Tlabel the $y$-axis such that $y\left(t_{0}\right) \leq 0$. The first thing to notice is that the retraction force is bounded by the force of the harmonic oscillator of the "momentary" frequency $\omega_{0}=x\left(t_{0}\right)$ as long as $y$ is negative. But as a harmonic oscillator returns to 0 within the next time interval of length $\pi / \omega_{0} \Gamma$ the $y$ motion has to cross the $x$ axis within this interval of time $\Gamma$ too. Let us call this moment of $y=0$ the time $t=0$.

Comparing with the harmonic oscillator once more $\mathrm{we}$ can conclude that the motion again is bounded from above by that of the harmonic oscillator and that $y$ will eventually return 0 at some moment $0<t_{1}<\pi / \omega_{0}$. 


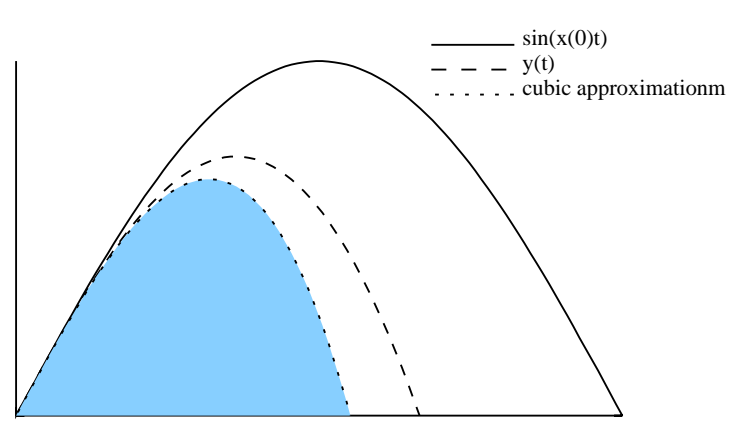

Fig. 29: Upper and lower bounds on $y(t)$

Now $\Gamma$ we are going to estimate the retarding effect of the oscillations in $y$ on the motion in $x$. Since we are going to show that the retardation is strong enough to stop the motion in $x$ we have to give a lower bound on $y$. At $t=0 \Gamma$ the velocity $\dot{y}$ is positive. Any trial function $\tilde{y}$ with $\tilde{y}(0)=0 \Gamma \dot{\tilde{y}}(0)=\dot{y}(0) \Gamma$ and $\ddot{\tilde{y}}(t)<\ddot{y}(t)$ will have this property. As the kinetic energy is bounded from above $\Gamma$ so is the velocity. Thus enough $\Gamma$ we know that $x(t)$ cannot double over one period. Hence $\Gamma$ with the help of the inequality

$$
-\ddot{y}(t) \leq 4 t \dot{y}(0) x(0)^{2}
$$

which holds because assuming the velocity in $y$ to be constant is an overestimate $\Gamma$ we can underestimate $y(t)$ by truncating its Taylor series at the cubic order

$$
y(t) \geq \sqrt{2 E_{y}} t-\frac{1}{3} \sqrt{2 E_{y}} x(0)^{2} t^{3} .
$$

Here $\Gamma$ we have used the equation of motion to see that there is no quadratic term in $t$. The right hand side is positive for $t<\sqrt{3} / x(0)$. Therefore $\Gamma$ we have the following underestimate for the deceleration in $x$ :

$$
\begin{aligned}
\int_{0}^{\sqrt{3} / x(0)} d t x(t) y(t)^{2} & >x(0) \int_{0}^{\sqrt{3} / x(0)} d t y(t)^{2} \\
& >2 E_{y} x(0) \int_{0}^{\sqrt{3} / x(0)} d t\left(t-\frac{1}{3} x(0)^{2} t^{3}\right)^{2} \\
& =\frac{16 \sqrt{3} E_{y}}{35 x(0)^{2}}
\end{aligned}
$$

This is the deceleration for one half of a quasi-cycle of the $y$ motion. We have to sum the contributions of all the cycles. At first sight $\Gamma$ this sum (which should be thought of as an integral over $x$ !) is convergent because the contribution is proportional to $1 / x^{2}$. But it is important to note that this contribution comes from the interval $\left[0, t_{1}\right]$ and its length is bounded by $\pi / x(0)$. Therefore $\Gamma$ the average value is bounded from below by

$$
\frac{16 \sqrt{3} E_{y}}{35 \pi x(0)}
$$


for the cycle beginning at $t=0$. To sum the contribution from all values of $x$ one has to sum a logarithmically divergent harmonic sum. This is in accordance with the logarithmic divergence we expected from the heuristic argument.

Hence we found that hhowever large $\dot{x}$ and however small the non-vanishing motion in $y$ is $\Gamma$ the motion in $x$ will eventually be stopped which is the contradiction we were looking for. Therefore $\mathrm{Wwe}$ can conclude that the only motions that reach infinity are those along straight lines.

\subsection{Scattering Solutions in the M(atrix)-Model}

The toy model enjoys one important simplification compared to the full M(atrix)-Model: There is no gauge freedom $\Gamma$ the valleys are along the $x$ - and $y$-axes whereas the global $S O(9) \times S U(N)$ symmetry allows one to rotate the valleys in any possible direction. The direction of the valleys is therefore dynamically determined and one should use appropriate variables to deal with this symmetry. Furthermore Tone could imagine a solution in which the matrices cannot asymptotically be brought to diagonal form because there is a nonvanishing motion in the $S U(N)$ directions that would destroy any choice of gauge at later times.

To approach these difficulties $\Gamma$ let us first discuss another simplified model that now contains all qualitative features of the full $\mathrm{M}$ (atrix)-Model; this allows one to translate the argument directly to the full M(atrix)-ModelГbut we prefer to present the approach in this model for notational simplicity.

The degrees of freedom are two two-vectors $\vec{X}_{a}(t) \in \mathbb{R}^{2}$ with $a=1,2$. The Lagrangian is given by (note the close similarity to the Lagrangian of the $S U(2)-\mathrm{M}($ atrix)Model!)

$$
L=\frac{1}{2}\left(\left\|\dot{\vec{X}}_{1}\right\|^{2}+\left\|\dot{\vec{X}}_{2}\right\|^{2}\right)-\left(\vec{X}_{1} \times \vec{X}_{2}\right)^{2}
$$

Again $\Gamma$ we ask whether there are solutions such that

$$
R^{2}:=\left\|\vec{X}_{1}\right\|^{2}+\left\|\vec{X}_{2}\right\|^{2}
$$

goes to infinity for early and late times. The form of the potential energy tells us that for large $\left\|\vec{X}_{1}\right\| \Gamma$ say $\Gamma$ the component of $\vec{X}_{2}$ that is perpendicular to $\vec{X}_{1}$ has to be small and oscillates with approximate frequency $\left\|\vec{X}_{1}\right\|$. Nevertheless $\Gamma$ it is not clear $\Gamma$ that there is an asymptotic direction for $\vec{X}_{1} \Gamma$ it might rotate around the origin forever. Therefore we cannot simply fix a gauge in which only one component of $\vec{X}_{2}$ plays the rôle of $y$ in the toy model.

To solve this problem $\Gamma$ the first observation is that $\Gamma$ just like the $\mathrm{M}$ (atrix)-Model $\Gamma$ this model not only has the obvious $S O(2)$ symmetry of vectors in $\mathbb{R}^{2}$ that parallels the 
$S U(N)$ symmetry of the M(atrix)-Model $\mathrm{Mbut}$ that it is invariant under another $S O(2)$ that acts on the $a$ index of the $\vec{X}_{a}$ Tbecause the potential is just the square of the determinant of the matrix $X_{a}{ }^{i}$. A parameterization that is adapted to the $S O(2) \times S O(2)$ symmetry is

$$
X_{a}{ }^{i}=\left(\begin{array}{cc}
\cos \alpha & \sin \alpha \\
-\sin \alpha & \cos \alpha
\end{array}\right)\left(\begin{array}{ll}
x & 0 \\
0 & y
\end{array}\right)\left(\begin{array}{cc}
\cos \beta & \sin \beta \\
-\sin \beta & \cos \beta
\end{array}\right) \text {. }
$$

If we rewrite the Lagrangian in these variables

$$
L=\frac{1}{2}\left(\dot{x}^{2}+\dot{y}^{2}+\left(x^{2}+y^{2}\right)\left(\dot{\alpha}^{2}+\dot{\beta}^{2}\right)+4 x y \dot{\alpha} \dot{\beta}\right)-x^{2} y^{2}
$$

we see that $\alpha$ and $\beta$ are cyclic variables and their conjugate momenta

$$
\begin{aligned}
& p_{\alpha}=\left(x^{2}+y^{2}\right) \dot{\alpha}+2 x y \dot{\beta} \\
& p_{\beta}=\left(x^{2}+y^{2}\right) \dot{\beta}+2 x y \dot{\alpha}
\end{aligned}
$$

are integrals of motion. We solve these for $\dot{\alpha}$ and $\dot{\beta}$ and use them to eliminate the $\alpha$ and $\beta$ dependence from the Lagrangian.

Then $\Gamma$ we arrive at the equation of motion for the remaining degrees of freedom

$$
\ddot{x}=-2 x y^{2}+\frac{\left(p_{\alpha}^{2}+p_{\beta}^{2}\right) x\left(x^{4}+10 x^{2} y^{2}+5 y^{4}\right)-2 p_{\alpha} p_{\beta} y\left(y^{4}+10 x^{2} y^{2}+5 x^{4}\right)}{\left(x^{2}-y^{2}\right)^{4}}
$$

and another one with $x \leftrightarrow y$. We use the same argument as before to show

$$
x>\frac{R}{\sqrt{2}}, \quad y<\frac{\sqrt{2} E}{R}
$$

which tells us that for large $R$ we have $x \sim R \gg y \sim 1 / R$. While the first term in (6.3) scales like $1 / R \Gamma$ the second term scales like $1 / R^{3}$ and can therefore be neglected for large $R$ :

$$
\ddot{x} \approx-2 x y^{2}+\frac{\left(p_{\alpha}^{2}+p_{\beta}^{2}\right) x^{5}-p_{\alpha} p_{\beta} y x^{4}}{x^{8}}
$$

In the numerator $\Gamma i t$ is not possible that the coefficient of $x^{5}$ vanishes while the coefficient of $y x^{4}$ is finite. Therefore $\Gamma$ for large enough $R$ (depending on the two angular momenta) $\Gamma$ we can neglect the second term in the numerator against the first. The fraction scales like $1 / R^{3}$ and is always strongly suppressed by the "toy-model" term.

Similarly $\Gamma$ for large $R \Gamma$ we have

$$
\ddot{y} \approx-2 y x^{2}+\frac{5\left(p_{\alpha}^{2}+p_{\beta}^{2}\right) x^{4} y-2 p_{\alpha} p_{\beta} x^{5}}{x^{8}} .
$$


The second term scales like $1 / R^{3} \Gamma$ whereas the first term scales like $y R^{2}$. As the amplitudes of the oscillations in $y$ are of the order $1 / R$ the second term will $\Gamma$ for most of the time of one oscillation $\Gamma$ be suppressed by four orders of $R$ and can therefore be neglected.

We have argued that in both non-trivial equations of motion $\Gamma$ the second terms can be neglected and in the limit of large $R \Gamma$ we are left with the toy model. In the previous section $\Gamma$ we proved that there are no non-trivial solution that can escape to infinity. Therefore there will be no scattering solutions for this modelГtoo.

The argument for the full $\mathrm{M}$ (atrix)-Modelshould be the same as above: One should rewrite the Lagrangian in terms of $S O(9) \times S U(N)$ angle variables and $N$ "radial" variables. In [SS] $\Gamma$ a corresponding gauge was fixed in which for one instant of time the M(atrix)-Model $X^{i}{ }_{a}$ were diagonal. Here we will do this "gauge fixing" dynamically by allowing the angles that determine the gauge to be time dependent degrees of freedom. These angles are again cyclic and can be eliminated whereas $\Gamma$ in the large $R$ limit $\Gamma$ the radial variables will be of the form of the toy model plus asymptotically small corrections.

Therefore $\Gamma$ it would be very suprising if there were non-trivial scattering solutions describing kinematics with momentum transfer in the finite $N \mathrm{M}$ (atrix)-Model. It seems that the finiteness of $N$ is very restrictive and puts strong constraints on the dynamics.

We should stress again that this result does not hold for infinite $N$ as there are the counterexamples we mentioned above[PP][KVK2][GHV]. The way to think about them is that once the number of degrees of freedom is infinite it is possible that an infinite number of them could each supply an infinitesimal amount of energy which finally pushes one of the "diagonal" degrees of freedom to infinity.

On the other hand $\Gamma$ these results about scattering solutions are welcome from the membrane point of view on the M(atrix)-Model: ThereГscattering solutions are to be considered degenerate $\Gamma$ they describe membranes whose shape at early and late times is completely dominated by one of the Fourier-modes of the world volume coordiante functions. The solutions that we classified as "bound states" from the D0 point of view describe membranes with oscillating shapes. Our result can be reformulated by saying that the latter are generic as one would have expected for fluctuating membranes. 


\section{Conclusions}

In this thesis $\Gamma$ we have investigated the $\mathrm{M}$ (atrix)-Model-conjecture that states that MTheory $\Gamma$ the Ur-theory that arises in the strong coupling limit of type IIA string theory is given $\Gamma$ in light-cone coordinates $\Gamma$ by the supersymmetric quantum mechanics of $S U(N)$ matrices. In particular $\Gamma$ we have tested Susskind's version of this conjecture that does not require the limit $N \rightarrow \infty$ and relates the finite $N$ version to the DLCQ of M-Theory.

Since eleven-dimensional supergravity is by definition the low-energy limit of MTheory it is a sensible question to ask if the M(atrix)-Model contains supergravity in the low-energy limit. A rich test-bed for such questions is provided by scattering processes. Scattering amplitudes and matrix elements of the $S$-matrix are natural and well-defined observables of a theory and we have calculated them using both theories $\Gamma$ the $\mathrm{M}($ atrix $)$ Model and supergravity.

Let us list the main results of this investigation:

- Besides the well-known result of leading-order scattering of two particles $\Gamma$ we have worked out the spin-spin coupling between the two particles. We have found full agreement with the spin-spin interactions of two gravitons in supergravity thus verifying that the particles described by the eigenvalues of the matrices in the M(atrix)-Model really have spin two as required by the conjecture. This result about a purely fermionic term in the model's effective action was later used in [PSS1] to prove a supersymmetric non-renormalization theorem for the $v^{4}$ term.

- We have been able to generalize the result of [OY1] on the scattering process of three particles by eliminating a kinematical constraint on the initial conditions that Okawa and Yoneya had to assume thereby putting the agreement between the supergravity prediction and the M(atrix)-Model result on a much wider basis.

- We have argued that the next-to-leading order of that three particle scattering amplitude in M(atrix)-Theory should be related to the first quantum correction of supergravity. We have worked out the amplitude on both sides and found a disagreement for finite $N$ not only in a numerical coefficient but in the tensor structure of the term in the effective action.

- We have discussed the possible origin of this disagreement.

- We have derived a no-go result for classical scattering solutions coming in from and leaving into the valleys of the potential that have non-trivial momentum transfer on the gauge theory side. The existence of such classical solutions is a pre-requisite for quantum scattering calculations that are beyond the restricted kinematics of the eikonal regime. This no-go result casts further doubt on the physical usefulness of the M(atrix)-Model-conjecture at finite $N$. 
We have already discussed possible explanations for the disagreement we found in the last section of chapter five. Here $\Gamma$ we would like to take a more general standpoint and consider the state of the M(atrix)-Model proposal in the view of our findings. In [T2] $\Gamma$ Taylor discusses several possibilities for the nature of M(atrix)-Theory that we would like to comment on:

(i) M(atrix)-Theory is correct $\Gamma$ and DLCQ supergravity is reproduced at finite $N$ perturbative M(atrix)-Theory calculations.

(ii) $\mathrm{M}$ (atrix)-Theory is correct in the large $N$ limit Tand non-perturbative supergravity is reproduced by a naïve large $N$ limit of the standard perturbative M(atrix)-Theory calculations.

(iii) $\mathrm{M}$ (atrix)-Theory is correct in the large $N$ limit $\Gamma$ but to connect it to supergravity even at the classical level it is necessary to deal with subtleties in the large $N$ limit (i.e. there are problems with the standard perturbative analysis at higher order).

(iv) $\mathrm{M}$ (atrix)-Theory is simply wrong Tand further terms need to be added to the dimensionally reduced super Yang-Mills action to find agreement with M-Theory even in the large $N$ limit.

Given the arguments by Sen and Seiberg we recapitulated in chapter three $\Gamma$ it is hard to believe in possibility (iv) although $\Gamma$ as we have pointed out $\Gamma$ all the agreement that was found in the past including the agreement we have presented here $\Gamma$ might be an artefact of the large amount of supersymmetry in the set-ups that were considered. We do not believe this is the case.

As we have explained our results on scattering amplitudes are still compatible with (i) as long as one has a very narrow definition of the terms used: One might be satisfied with an agreement with classical supergravity We do not think this is a viable optionTas the primary purpose of string theory is to give a unified description of nature. To the best of today's knowledge this "theory of everything" has to include a quantum theory of gravity. As we have seen $\Gamma$ correct implementation of the DLCQ is very subtle $\Gamma$ especially in a quantum theory. It might be completely impossible to extrapolate results obtained in a DLCQ limit to an uncompactified version of the theory much different to the situation in spatially compactified theories that become uncompactified in a large volume limit.

On the other hand our findings about the impossibility to transfer momentum in classical scattering processes appears to be in conflict with possibility (i) as it seems that the finite $N$ kinematics accessible to $\mathrm{M}$ (atrix)-Theory are severely restricted.

On the basis of our investigations alone $\Gamma$ we cannot differentiate between possibility (ii) and (iii) since we have strictly stuck to finite $N$ calculations. Both seem equally likely. But given today's large $N$ technology $\Gamma$ this means that the usefulness of the M(atrix)Theory-conjecture is rather restricted. What would be needed are several achievements: 
First $\Gamma$ more information about the structure of the ground state that describes a graviton with $N>1$ Tideally the exact wave functionm $\Gamma$ is needed. It should be expected that scattering amplitudes at large $N$ depend for example on the distributions of velocities of the different "partons" within the graviton.

Then a formalism to extract the leading large $N$ behavior is needed. In the best of all worlds $\Gamma$ this would be some kind of large $N$ renormalization group: A way to integrate out the contributions of single rows and columns of the matrices that could relate the theory at some $N$ to a theory with $N-1$. Both these requirements are beyond reach given today's techniques.

So far $\Gamma \mathrm{M}$ (atrix)-Theory has only been successfully applied to M-Theory fluctuations around a flat possibly toroidally compactified $\Gamma$ background. There are indications[DOS][DO] that for curved backgrounds a model with finitely many degrees of freedom is probably not sufficient to reproduce the appropriate amplitudes. Only very limited work in this direction has been done and much more is needed.

It would also be fruitful to investigate the importance of supersymmetry in the relation between supergravity and gauge theory. A possible calculation to approach this problem would be to check the correspondence between supergravity and M(atrix)-Theory at the eighth order in velocities. As we have explained before $\Gamma$ at that order $\Gamma$ supersymmetry is very unlikely to protect the amplitude while supergravity appears still at the classical level. Therefore $\Gamma$ this calculation would allow for a clear-cut distinction between disagreement because of lack of supersymmetry and disagreement because of subtleties of the DLCQ limit. We leave this technical tour de force for future investigations. 


\section{Acknowledgments}

I have the pleasure to thank the many people without whom this work would have never been possible. First of all Tthere is Hermann Nicolai. His advice judgment and motivation have always been of paramount importance to me. I have benefited very much from the atmosphere he creates in his quantum gravity group. Besides HermannГI would like to thank my other collaborators whom it was a pleasure to tackle physics problems with: An-

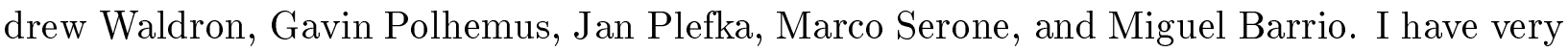
much enjoyed discussing physics also with Alan Rendall $\Gamma$ Andreas Recknagel $\Gamma$ Dieter Lüst $\Gamma$ Germar SchröderГHenning RehrenГHenning SamtlebenГIlka BrunnerГJens HoppeГKilian KoepsellГMarkus PösselГMatthias StaudacherГMax ZuckerГMichael Douglas $\Gamma$ Sebastian Silva Tamas Hauer and Volker Schomerus. I am also very much indebted to Andreas for a very careful reading of this manuscript. My colleagues at the Albert-Einstein-Institut at Potsdam and later at Golm have made my time a great one! I am grateful to the MaxPlanck-Society that has provided me with financial support. Last but not least I would like to express my gratitude to my parents and Annette Kaufmann for the stimulation and support they have continously been giving. 
8. Bibliography

[AdWLN] Mohab Abou-Zeid and Bernard de Wit and Dieter Lüst and Hermann Nicolai: "Space-Time SupersymmetryГIIA/B Duality And M-Theory" $\Gamma$ Phys. Lett. B466 (1999) p. 144 hep-th/9908169

[AMRV] I. Ya. Aref'eva and P. B. Medvedev and O. A. Rytchkov and I. V. Volovich: "Chaos in M(atrix) Theory" Thep-th/9710032

[AS] P. C. Aichelburg and R. U. Sexl: "On The Gravitational Field Of A Massless Particle" TGen. Rel. Grav. 2 (1971) p. 303-312

[Ba] Tom Banks: "TASI Lectures on Matrix Theory" $\Gamma h e p-t h / 9911068$

[BB] Katrin Becker and Melanie Becker: "A Two Loop Test of M(atrix) Theory" $\Gamma$ Nucl. Phys. B506 (1997) p. 48 hep-th/9705091

[BBPT] Katrin Becker and Melanie Becker and Joseph Polchinski and Arkady Tseytlin: "Higher Order Graviton Scattering in M(atrix) Theory" ГPhys. Rev. D56 (1997) p. $3174-3178$ hep-th/9706072

[BFSS] Tom Banks and Willy Fischler and Steven H. Shenker and Leonard Susskind: "M-Theory as A Matrix Model: A Conjecture" $\Gamma$ Phys. Rev. D55 (1997) p. 5112-5128 hep-th/9610043

[BHP] Miguel Barrio and Robert Helling and Gavin Polhemus: "Spin-Spin Interaction in Matrix Theory" ГJHEP 05 (1998) p. 012 hep-th/9801189

[Bi] Adel Bilal: "A Comment On Compactification of M-Theory On An (Almost) Light-Like Circle" $\Gamma$ Nucl. Phys. B521 (1998) p. 202 hep-th/9801047

[BMM] T. S. Biró and S. G. Matinyan and B. Müller : "Chaos and Gauge Field Theory" (1994) World Scientific

[BMS] Martin Bordemann and Eckhard Meinrenken and Martin Schlichenmaier: "Toep-

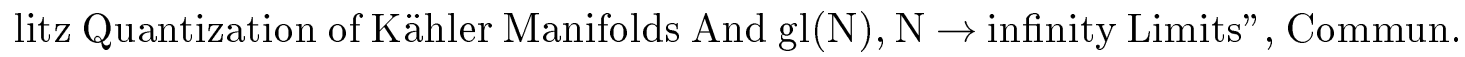
Math. Phys. 165 (1994) p. 281-296 hep-th/9309134

[BRR] M. Baake and M. Reinicke and V. Rittenberg: "Fierz Identities For Real Clifford Algebras And The Number Of Supercharges" Г J. Math. Phys. 26 (1985) p. 1070 
[BS] Daniela Bigatti and Leonard Susskind: "Review of Matrix Theory" $\Gamma$ hep-th/ 9712072

[BST] E. Bergshoeff and E. Sezgin and P. K. Townsend: "Properties Of The ElevenDimensional Super Membrane Theory" TAnn. Phys. 185 (1988) p. 330

[CH] Mark Claudson and Martin B. Halpern: "Supersymmetric Ground State Wave Functions" TNucl. Phys. B250 (1985) p. 689

[CJ] Eugene Cremmer and Bernard Julia and Joel Scherk: "Supergravity Theory in 11 Dimensions" TPhys. Lett. B76 (1978) p. 409-412

[DEG] Michael Dine and Robert Echols and Joshua P. Gray: "Renormalization of Higher Derivative Operators in The Matrix Model" ГPhys. Lett. B444 (1998) p. 103 hep-th/9805007

[DKPS] Michael R. Douglas and Daniel Kabat and Philippe Pouliot and Stephen H. Shenker: "D-branes And Short Distances in String Theory" $\Gamma$ Nucl. Phys. B485 (1997) p. 85-127 hep-th/9608024

[DO] Michael R. Douglas and Hirosi Ooguri: "Why Matrix Theory Is Hard" $\Gamma$ Phys. Lett. B425 (1998) p. 71 hep-th/9710178

[DOS] Michael R. Douglas and Hirosi Ooguri and Stephen H. Shenker : "Issues in (M)atrix Model Compactification" $\Gamma$ Phys. Lett. B402 (1997) p. 36-42 hepth/9702203

[DR] Michael Dine and Arvind Rajaraman: "Multigraviton Scattering in The Matrix Model" ГPhys. Lett. B425 (1998) p. 77 hep-th/9710174

[DVV] Robbert Dijkgraaf and Erik Verlinde and Herman Verlinde: "Matrix String Theory" TNucl. Phys." Tî volume = "B500 (1997) p. 43 hep-th/9703030

[dWHN] Bernard de Wit and Jens Hoppe and Hermann Nicolai: "On The Quantum Mechanics Of Supermembranes" TNucl. Phys. B305 (1988) p. 545

[dWLN] B. de Wit and M. Luscher and H. Nicolai: "The Supermembrane Is Unstable" $\Gamma$ Nucl. Phys. B320 (1989) p. 135

[F] R. Flume: "On Quantum Mechanics With Extended Supersymmetry And Nonabelian Gauge Constraints" ГAnn. Phys. 164 (1985) p. 189

[Fey] Richard P. Feynman and Albert R. Hibbs: "Quantum Mechanics And Path Integrals" (1965) MacGraw-Hill

[FFI] Marco Fabbrichesi and Gabriele Ferretti and Roberto Iengo: "About The Matrix Model Computation of Three-Graviton Scattering" Thep-th/9806166 
[FGHHY] J. Fröhlich and G. M. Graf and D. Hasler and J. Hoppe and S. T. Yau: "Asymptotic Form of Zero Energy Wave Functions in Supersymmetric Matrix Models" $\Gamma$ hep-th/9904182

[FH] Jürg Fröhlich and Jens Hoppe: "On Zero-Mass Ground States in Super-Membrane Matrix Models" $\Gamma$ Commun. Math. Phys. 191 (1998) p. 613 hep-th/ 9701119

[FT] E. S. Fradkin and A. A. Tseytlin: "Quantum Properties Of Higher Dimensional And Dimensionally Reduced Supersymmetric Theories" $\Gamma$ Nucl. Phys. B227 (1983) p. 252

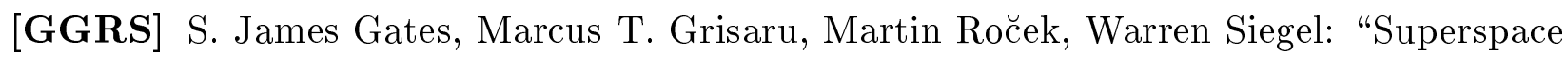
or One Thousand and One Lessons in Supersymmetry" (1983) Benjamin/Cummings

[GGV] Michael B. Green and Michael Gutperle and Pierre Vanhove: "One Loop in Eleven Dimensions" $\Gamma$ Phys. Lett. B409 (1997) p. 177-184 hep-th/9706175

[GHV] Steven B. Giddings and Feike Hacquebord and Herman Verlinde : "High Energy Scattering And D-pair Creation in Matrix String Theory" $\Gamma$ Nucl. Phys. B537 (1999) p. 260 hep-th/9804121

[GSB] Michael B. Green and John H. Schwarz and Lars Brink: "N=4 Yang-Mills And N=8 Supergravity As Limits Of String Theories" $\Gamma$ Nucl. Phys. B198 (1982) p. 474-492

[GSW] Michael B. Green and John H. Schwarz and Edward Witten: "Superstring Theory" (1987) Cambridge University Press

[H] Jeffrey A. Harvey: "Spin Dependence of D0-brane Interactions" $\Gamma$ Nucl. Phys. Proc. Suppl. 68 (1998) p. 113 hep-th/9706039

[HKS] Seungjoon Hyun and Youngjai Kiem and Hyeonjoon Shin: "Supersymmetric Completion of Supersymmetric Quantum Mechanics" $\Gamma$ Nucl. Phys. B558 (1999) p. 349 hep-th/9903022

[Ho] Jens Hoppe: "Quantum Theory of A Massless Relativistic Surface And A TwoDimensional Bound State Problem" (1982) MIT PhD-Thesis

[HP] Simeon Hellerman and Joseph Polchinski: "Compactification in The Light-Like Limit" ГPhys. Rev. D59 (1999) p. 125002 hep-th/9711037

[HPSW] Robert Helling and Jan Plefka and Marco Serone and Andrew Waldron: "Three Graviton Scattering in M-Theory" TNucl. Phys. B559 (1999) p. 184-204 hepth/9905183 
[HS] M. B. Halpern and C. Schwartz: "Asymptotic Search For Ground States of SU(2) Matrix Theory" ГInt. J. Mod. Phys. A13 (1998) p. 4367-4408 hep-th/9712133

[HT] Chris M. Hull and Paul K. Townsend: "Enhanced Gauge Symmetries in Superstring Theories" TNucl. Phys. B451 (1995) p. 525-546 hep-th/9505073

[IMSY] Nissan Itzhaki and Juan M. Maldacena and Jacob Sonnenschein and Shimon Yankielowicz: "Supergravity And The Large N Limit Of Theories With Sixteen Supercharges" TPhys. Rev. D58 (1998) p. 046004 hep-th/9802042

[K] Per Kraus: "Spin-Orbit Interaction From Matrix Theory" $\Gamma$ Phys. Lett. B419 (1998) p. 73 hep-th/9709199

[KNS] Werner Krauth and Hermann Nicolai and Matthias Staudacher: "Monte Carlo Approach to M-Theory" TPhys. Lett. B431 (1998) p. 31-41 hep-th/9803117

[KS] Victor G. Kac and Andrei V. Smilga: "Normalized Vacuum States in N = 4 Supersymmetric Yang-Mills Quantum Mechanics with Any Gauge Group" Thepth/9908096

[KVK1] Esko Keski-Vakkuri and Per Kraus: "Short Distance Contributions to Graviton Graviton Scattering: Matrix Theory Versus Supergravity" $\Gamma$ Nucl. Phys. B529 (1998) p. 246 hep-th/9712013

[KVK2] Esko Keski-Vakkuri and Per Kraus: "M-Momentum Transfer Between Gravitons Membranes And Five- Branes As Perturbative Gauge Theory Processes" $\Gamma$ Nucl. Phys. B530 (1998) p. 137 hep-th/9804067

[LT] Dieter Lüst and Stefan Theisen: "Lectures on String Theory" (1989) Springer

[McA] Ian N. McArthur: "Higher Order Spin-Dependent Terms in D0-brane Scattering from The Matrix Model" TNucl. Phys. B534 (1998) p. 183 hep-th/9806082

[MNS] Gregory Moore and Nikita Nekrasov and Samson Shatashvili: "D-particle Bound States And Generalized Instantons" TCommun. Math. Phys. 209 (2000) p. 77 hep-th/9803265

[MSS] Jose F. Morales and Claudio A. Scrucca and Marco Serone: "Scale Independent Spin Effects in D-brane Dynamics" $\Gamma$ Nucl. Phys. B534 (1998) p. 223 hepth/9801183

[NH] Hermann Nicolai and Robert Helling: "Supermembranes and M(atrix) Theory" $\Gamma$ hep-th/9809103

[NP1] Hermann Nicolai and Jan Plefka: "A Note on The Supersymmetric Effective Action of Matrix Theory" Thep-th/0001106 
[NP2] Hermann Nicolai and Jan Plefka: unpublished

[OY1] Yuji Okawa and Tamiaki Yoneya: "Multi-body Interactions of D-particles in Supergravity And Matrix Theory" $\Gamma$ Nucl. Phys. B538 (1999) p. 67 hepth/9806108

[OY2] Yuji Okawa and Tamiaki Yoneya: "Equations of Motion And Galilei Invariance in D-particle Dynamics" TNucl. Phys. B541 (1999) p. 163 hep-th/9808188

[P1] Joseph Polchinski: "Dirichlet-Branes and Ramond-Ramond Charges" $\Gamma$ Phys. Rev. Lett. 75 (1995) p. 4724-4727 hep-th/9510017

[P2] Joseph Polchinski: "String Theory" (1998) Cambridge University Press

[PP] Joseph Polchinski and Philippe Pouliot: "Membrane Scattering With M-momentum Transfer" ГPhys. Rev. D56 (1997) p. 6601-6606 hep-th/9704029

[PR] Massimo Porrati and Alexander Rozenberg: "Bound States at Threshold in Supersymmetric Quantum Mechanics" $\Gamma$ Nucl. Phys. B515 (1998) p. 184 hepth/9708119

[PSS1] Sonia Paban and Savdeep Sethi and Mark Stern: "Constraints from Extended Supersymmetry in Quantum Mechanics" TNucl. Phys. B534 (1998) p. 137 hepth/9805018

[PSS2] Sonia Paban and Savdeep Sethi and Mark Stern: "Supersymmetry And Higher Derivative Terms in The Effective Action of Yang-Mills Theories" $\Gamma$ JHEP 06 (1998) p. 012 hep-th/9806028

[PSW] Jan C. Plefka and Marco Serone and Andrew K. Waldron: "The Matrix Theory S-Matrix" TPhys. Rev. Lett. 81 (1998) p. 2866-2869 hep-th/9806081

[RT] John G. Russo and Arkady A. Tseytlin: "One-Loop Four-Graviton Amplitude in Eleven-Dimensional Supergravity" $\Gamma$ Nucl. Phys. B508 (1997) p. 245 hepth/9707134

[Sa] Sigurd Sannan: "Gravity As The Limit of The Type II Superstring Theory" $\Gamma$ Phys. Rev. D34 (1986) p. 1749

[Sch] Julian Schwinger: "On Gauge Invariance And Vacuum Polarization" TPhys. Rev. 82 (1951) p. 664-679

[Se] Ashoke Sen: "D0-branes on T(n) And Matrix Theory" $\Gamma$ Adv. Theor. Math. Phys. 2 (1998) p. 51 hep-th/9709220

[Sei] Nathan Seiberg: "Why Is The Matrix Model Correct?" $\Gamma$ Phys. Rev. Lett. 79 (1997) p. 3577-3580 hep-th/9710009 
[Ser] Marco Serone: "A Comment on The $R^{4}$-Coupling in (M)atrix Theory" $\Gamma$ Phys. Lett. B422 (1998) p. 88 hep-th/9711031

[Sm] Andrei V. Smilga: "Witten Index Calculation In Supersymmetric Gauge Theory" TNucl. Phys. B266 (1986) p. 45-57

[SS] Savdeep Sethi and Mark Stern: "D-brane Bound States Redux" $\Gamma$ Commun. Math. Phys. 194 (1998) p. 675 hep-th/9705046

[ST] N. Sakai and Y. Tanii: "One Loop Amplitudes And Effective Action In Superstring Theories" TNucl. Phys. B287 (1987) p. 457

[Su] Leonard Susskind: "Another Conjecture about M(atrix) Theory" Thep-th/97040 80

[T] Paul K. Townsend: "M-Theory from Its Superalgebra" Thep-th/9712004

[T1] Washington TaylorГIV: "D-brane Field Theory on Compact Spaces" ГPhys. Lett. B394 (1997) p. 283-287 hep-th/9611042

[T2] Washington TaylorГIV: "The M(atrix) Model of M-Theory" Thep-th/0002016

[TvR] Washington Taylor IV and Mark Van Raamsdonk: "Three-Graviton Scattering in Matrix Theory Revisited" ГPhys. Lett. B438 (1998) p. 248 hep-th/9806066

[V] Jos A. M. Vermaseren: "Symbolic manipulation with FORM" (1991) Computer Algebra Netherland

[Ve] G. Veneziano: "Construction of A Crossing-Symmetric $\Gamma R e g g e$ Behaved Amplitude For Linearly Rising Trajectories" $\Gamma$ Nuovo Cim. 57A (1968) p. 190

[W] Edward Witten: "String Theory Dynamics in Various Dimensions" $\Gamma$ Nucl. Phys. B443 (1995) p. 85-126 hep-th/9503124

[Y] Piljin Yi: "Witten Index And Threshold Bound States of D-branes" $\Gamma$ Nucl. Phys. B505 (1997) p. 307 hep-th/9704098

[Z] A. H. Zemanian: "Distribution Theory and Transform Analysis" (1965) Dover Publications 


\section{Erklärung}

Hiermit versichere ichГdie vorliegende Arbeit selbständig angefertigt zu haben und keine weiteren als die angegebenen Hilfsmittel verwendet zu haben. 